\title{
DISCOVERY AND CHARACTERIZATION OF A HIGGS-LIKE RESONANCE USING THE MATRIX ELEMENT LIKELIHOOD APPROACH
}

by

\author{
Andrew J. Whitbeck \\ A dissertation submitted to The Johns Hopkins University in conformity with the \\ requirements for the degree of Doctor of Philosophy. \\ Baltimore, Maryland
}

September, 2013

(c) Andrew J. Whitbeck 2013

All rights reserved 


\section{Abstract}

Understanding the exact mechanism of electroweak symmetry breaking through the discovery and characterization of the Higgs boson is one of the primary goals of the Large Hadron Collider (LHC). Two searches for a Higgs boson decaying to a pair of $\mathrm{Z}$ bosons with subsequent decays to either $2 \ell 2 q$ or $4 \ell$ are presented using data recorded with the Compact Muon Solenoid (CMS). The discovery and characterization of a Higgs-like resonance using a new set of tools is reported. The foundations of such tools are developed and prospects for their use in other Higgs channels and at future colliders are addressed. Although the Standard Model (SM) of electroweak interactions has been extremely successful in describing a number of phenomena, there are still questions to be addressed pertaining to its naturalness and its possible con-

nection to beyond the SM physics. Results are interpreted in the context of possible extensions to the SM and their effect on our understanding of the universe.

Primary Reader: Andrei Gritsan

Second Reader: Barry Blumenfeld 
ABSTRACT 


\section{Acknowledgments}

I would like to thank Andrei Gritsan for accepting me as a student. I am lucky to have been a part of developing the great ideas that have resulted from his research program and have learned an immense amount physics and how to approach research problems. I have been fortunate to take on a leading role in my field and to represent my collaboration on more than one occasion as an ambassador to the greater scientific community. This would not have been possible without his encouragement and guidance.

I would also like to thank everyone involved with CMS and the LHC. It has been a remarkable experience to be a part of the collaboration and see what can be done when thousands of people put their minds to one big idea. I would also like to give special thanks to all the CMS research groups: the Higgs PAG, HZZ subgroup, and tracker alignment group. I am eternally grateful for those who have supported me in my continue academic career: Chiara, Joe, Andrey, and Yves. 


\section{Contents}

Abstract

Acknowledgments $\quad$ iv

List of Tables $\quad$ ix

List of Figures $\quad$ xi

1 Introduction 1

1.1 Electroweak Symmetry Breaking . . . . . . . . . . . . . . 4

1.2 Higgs Boson Constraints . . . . . . . . . . . . . . . . . 5

1.3 Beyond the SM Higgs . . . . . . . . . . . . . . . . . 6

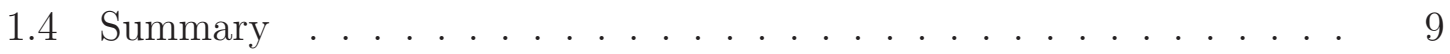

2 Experimental Setup $\quad 10$

2.1 The Large Hadron Collider . . . . . . . . . . . . . . . . . . . . 10

2.2 The Compact Muon Solenoid . . . . . . . . . . . . . . . . . . . 12 


\section{CONTENTS}

2.2.1 Magnet ..................... . . . 13

2.2.2 Trigger and data acquisition . . . . . . . . . . . . . 14

2.2.3 Electromagnetic Calorimeter . . . . . . . . . . . . . 14

2.2.4 Hadronic Calorimeter . . . . . . . . . . . . . . . . . . 15

2.2.5 Muon System . . . . . . . . . . . . . . . . . . . . 17

2.2 .6 Tracker .......................... 18

2.2.6.1 Pixel Modules . . . . . . . . . . . . . . . . . . 19

2.2.6.2 Strip Modules . . . . . . . . . . . . . . . . . . 19

2.2.6.3 Tracking Performance \& Alignment . . . . . . . . . . 21

2.3 Summary . . . . . . . . . . . . . . . . . . . 30

3 Higgs Phenomenology at the LHC 32

3.1 Higgs Signatures . . . . . . . . . . . . . . . . . 33

3.1.1 Gluon-gluon Fusion . . . . . . . . . . . . . . . . . . . . . 33

3.1 .2 Weak Vector Boson Fusion . . . . . . . . . . . . . . . . . 34

3.1.3 Other Production Mechanisms . . . . . . . . . . . . . 35

3.1.4 Decay Channels . . . . . . . . . . . . . . . . 36

3.2 Kinematics of Scalar Resonances . . . . . . . . . . . . . . 37

3.2.1 Variables for Property Measurements . . . . . . . . . . . . . . 43

3.3 Summary . . . . . . . . . . . . . . . . . . . . 51

4 Higgs Searches with ZZ decays $\quad 52$ 


\section{CONTENTS}

4.1 Semi-leptonic decay channel . . . . . . . . . . . . . . . . . . . 54

4.1.1 Event Simulation . . . . . . . . . . . . . . 54

4.1.2 Event Reconstruction, Selection, and Categorization . . . . . . 55

4.1.3 Yields and Kinematics Distributions . . . . . . . . . . . . 61

4.1.4 Results of Semilepton Analysis . . . . . . . . . . . . . . . . 72

4.2 Golden Decay Channel . . . . . . . . . . . . . . . . . . . 75

4.2.1 Datasets .................... . . 75

4.2.2 Event Selection and Categorization . . . . . . . . . . . . 77

4.2.3 Yields and Kinematics Distributions . . . . . . . . . . . 78

4.2.4 Observation ....................... 82

4.2.5 Spin and Parity Measurements . . . . . . . . . . . . . . 87

4.2.6 Constraining CP-violation . . . . . . . . . . . . . . . . . 93

4.3 Summary . . . . . . . . . . . . . . . . . . . . . 95

5 Future Measurements 102

5.1 Multidimensional Fits . . . . . . . . . . . . . . . . 103

5.2 LHC Projections . . . . . . . . . . . . . . . . . . . 109

5.3 Future Colliders . . . . . . . . . . . . . . . . . . . . . . . 112

5.4 Other Channels . . . . . . . . . . . . . . . . . 117

5.5 Summary . . . . . . . . . . . . . . . . . . . . 118

6 Conclusions $\quad 119$ 


\section{CONTENTS}

Bibliography

126

Vita

139 


\section{List of Tables}

1.1 List of SM particles and their charges. Q represents the charge of the $S U(1)_{\text {em }}$ gauge symmetry, $T_{3}$ the broken SU(2) gauge symmetry, and color the charge of the $S U(3)$ gauge symmetry. . . . . . . . . . .

2.1 Relevant operational LHC parameters and there values at under design conditions. . . . . . . . . . . . . . . . .

3.1 List of alternative signal models to be tested against the SM Higgs hypothesis along with a description of the their couplings to ZZ. Amplitude parametrization for spin-0 resonances is given in Equation 3.1; parametrizations for spin-1 and spin-2 resonances are given in Equations 16 and 18 elsewhere [1]. . . . . . . . . . . . . . .

4.1 Table summarizing MC simulations used to model signal and each of the different SM background along with their cross sections. . . . . .

4.2 Table listing analysis selections. The top portion details preselection cuts applied to all objects to be consistent with trigger requirements and detector acceptance. The bottom portion details all cuts applied in each of the different b-tag categories to optimize the sensitivity to signal events. . . . . . . . . . . . . . . . . . .

4.3 Summary of systematic uncertainties on signal normalization. Most sources give multiplicative uncertainties on the cross section measurement, except for the expected Higgs boson production cross section, which is relevant for the measurement of the ratio to the SM expectation. The ranges indicate dependence on $m_{H} \ldots \ldots$. . . . . . 


\section{LIST OF TABLES}

4.4 Observed and expected event yields for $4.6 \mathrm{fb}^{-1}$ of data. The yields are quoted in the ranges $125<m_{Z Z}<170 \mathrm{GeV}$ or $183<m_{Z Z}<$ $800 \mathrm{GeV}$, depending on the Higgs boson hypothesis. The expected background is quoted from both the data-driven estimations and from $\mathrm{MC}$ simulations directly. In the low-mass range, the background is estimated from the $m_{Z Z}$ sideband for each Higgs mass hypothesis and is not quoted in the table. The errors on the expected background from simulation include only statistical uncertainties. . . . . . . . . .

4.5 List of MC samples used for the $Z Z^{(*)} \rightarrow 4 \ell$ analysis. along with the event generator used to simulate them. . . . . . . . . . . . .

4.6 Expected and observed yields in the mass range $121.5<m_{4 \ell}<130.5$ for different event classes. . . . . . . . . . . . . . . . .

4.7 Expected and observed yields in the mass range $100<m_{4 \ell}<1000$ for difference class of events. . . . . . . . . . . . . . . . . . .

4.8 Table with correction factors and event yields in the different channels of the alternative spin-0 hypotheses arising due to lepton interference and detector effects. . . . . . . . . . . . . . . . . . . .

4.9 Table with correction factors and event yields in the different channels of the alternative spin-1 hypotheses arising due to lepton interference and detector effects. . . . . . . . . . . . . . . . .

4.10 Table with correction factors and event yields in the different channels of the alternative spin- 2 hypotheses with minimal couplings arising due to lepton interference and detector effects. . . . . . . . . . . . .

4.11 Table with correction factors and event yields in the different channels of the alternative spin-2 hypotheses with high dimensional couplings arising due to lepton interference and detector effects. . . . . . . . . .

4.12 List of models used in analysis of spin-parity hypotheses corresponding to the pure states of the type noted. The expected separation is quoted for two scenarios, when the signal strength for each hypothesis is predetermined from the fit to data and when events are generated with SM expectation for the signal yield $(\mu=1)$. The observed separation quotes consistency of the observation with the $0^{+}$model or $J^{P}$ model, and corresponds to the scenario when the signal strength is pre-determined from the fit to data. The last column quotes $\mathrm{CL}_{s}$ criterion for the $J^{P}$ model.

5.1 List of cross sections and event yields for Higgs production and decay processes. . . . . . . . . . . . . . . . . . 110

5.2 List of $f_{a 3}$ values for various processes. . . . . . . . . . . 115 


\section{List of Figures}

1.1 Feynman diagram depicting electron-electron scattering via the electromagnetic interaction. . . . . . . . . . . . . . . . .

1.2 Constraints on the SM Higgs boson mass from Tevatron and LEP experiments either through direct searches or indirect evidence based on precision measurements. . . . . . . . . . . . . . . . . .

2.1 Quarter slice of the CMS tracker. Single-sided silicon strip modules are indicated as solid light (purple) lines, double-sided strip modules as open (blue) lines, and pixel modules as solid dark (blue) lines. . . .

2.2 Diagram of module position variables, $\mathrm{u}, \mathrm{v}, \mathrm{w}$, and module orientation variables, $\alpha, \beta, \gamma \ldots \ldots \ldots \ldots$

2.3 Resolution of 5 track parameters from track splitting validation using three geometries, ideal (blue), prompt geometry (black), and the aligned geometry (red). Cosmic track recording during the 2012 Run A period were used. . . . . . . . . . . . . . . .

2.4 Profile plots of several reference geometries using cosmic tracks recorded during the 2012 Run A period. The left plot shows the difference in $d_{x y}$ between the two split tracks, $\Delta d_{x y}$ vs $\phi$. The right plot shows the width of the $\Delta p_{T}$ distribution, $\sigma\left(p_{T}\right)$, vs $p_{T} \ldots \ldots \ldots$

2.5 Diagram depicting the calculation of residuals used in the primary vertex validation. . . . . . . . . . . . . . . . .

2.6 Residual transverse impact parameter distributions in bins of $\eta$ (top) and $\phi$ (bottom) . . . . . . . . . . . . . . . . .

2.7 Distribution of mean and width of transverse impact parameter residuals in bins of the probe tracks azimuthal angle, $\phi$, for an ideal geometry (black), ideal geometry plus $40 \mu \mathrm{m}$ separation between the pixel half barrels (red), and the 2011 candidate geometry (blue). . . . . . . . .

2.8 Measured separation between pixel half barrels versus time before and after alignment. . . . . . . . . . . . . . . . 


\section{LIST OF FIGURES}

3.1 left: Higgs production cross section vs $m_{H}$ for different processes at $\sqrt{s}=8 \mathrm{TeV}$. right: Higgs branching ratios vs $m_{H}$. Both calculations are taken from the LHC Higgs cross section working group. . . . . .

3.2 Distribution of parton factor, $\mathrm{F}(\mathrm{s}, \mathrm{Y}=0)$, showing the relative probability for producing resonances from gluon-gluon, or $q \bar{q}$ interaction for $\sqrt{s}=14 \mathrm{TeV} \ldots \ldots \ldots \ldots \ldots \ldots$

3.3 Feynman diagram depicting the leading contribution to gluon-gluon fusion production of a Higgs boson. . . . . . . . . . . . . . . .

3.4 Feynman diagram depicting weak vector boson fusion production of a Higgs boson. . . . . . . . . . . . . . . . . . . . .

3.5 Feynman diagram depicting associated production (left) and $t \bar{t}$ fusion production of a Higgs boson. . . . . . . . . . . . . . . . . . . . .

3.6 Diagram depicting $H \rightarrow Z Z \rightarrow 4 \ell$ decays and definition of angles which describe the kinematics of these decays. . . . . . . . . . .

3.7 Distributions of the $\mathrm{Z}$ boson masses. The smaller of the two masses is plotted on the right, while the larger of the two masses is plotted on the left. Markers show simulated events; lines are projections of the analytical distribution described above. Red lines/circles correspond to a SM Higgs, blue lines/diamonds, a pseudoscalar, and green lines/square, a CP-even scalar produced from higher dimension operators. . . . . . .

3.8 Distributions of helicity angles, $\cos \theta_{1}$ (left), $\cos \theta_{2}$ (middle), and $\Phi$ (right). Markers show simulated events; lines are projections of the analytical distribution described above. Red lines/circles correspond to a SM Higgs, blue lines/diamonds, a pseudoscalar, and green lines/square, a CP-even scalar produced from higher dimension operators. . . . . .

3.9 Distributions of the $\mathrm{Z}$ boson masses. The smaller of the two masses is plotted on the right, while the larger of the two masses is plotted on the left. Markers show simulated events; lines are projections of the analytical distribution described above. Red lines/circles correspond to a CP-even vector, blue lines/diamonds to a CP-odd vector. . . . .

3.10 Distributions of the production angles, $\cos \theta^{*}$ (left) and $\Phi_{1}$ (right). Markers show simulated events; lines are projections of the analytical distribution described above. Red lines/circles correspond to CP-even vector, blue lines/diamonds to a $\mathrm{CP}$-odd vector. . . . . . . . . . . .

3.11 Distributions of the helicity angles, $\cos \theta_{1}$ (left), $\cos \theta_{2}$ (middle), and $\Phi$ (right). Markers show simulated events; lines are projections of the analytical distribution described above. Red lines/circles correspond to $\mathrm{CP}$-even vector, blue lines/diamonds to a $\mathrm{CP}$-odd vector. . . . . 


\section{LIST OF FIGURES}

3.12 Distributions of the $\mathrm{Z}$ boson masses. The smaller of the two masses is plotted on the right, while the larger of the two masses is plotted on the left. Markers show simulated events; lines are projections of the analytical distribution described above. Red lines/circles correspond to a minimal coupling graviton, blue lines/diamonds to a CP-odd tensor, and green lines/square to a CP-even tensor produced from higher dimension operators. . . . . . . . . . . . . . . . . .

3.13 Distributions of the production angles, $\cos \theta^{*}$ (left) and $\Phi_{1}$ (right). Markers show simulated events; lines are projections of the analytical distribution described above. Red lines/circles correspond to a minimal coupling graviton, blue lines/diamonds to a CP-odd tensor, and green lines/square to a CP-even tensor produced from higher dimension operators. . . . . . . . . . . . . . . . . .

3.14 Distributions of the helicity angles, $\cos \theta_{1}$ (left), $\cos \theta_{2}$ (middle), and $\Phi$ (right). Markers show simulated events; lines are projections of the analytical distribution described above. Red lines/circles correspond to a minimal coupling graviton, blue lines/diamonds to a CP-odd tensor, and green lines/square to a CP-even tensor produced from higher dimension operators. . . . . . . . . . . . . . . .

3.15 Distributions of $D_{0-}$ (left) and $D_{0_{h}^{+}}$(right) for various scalar models. A SM Higgs (open red circles), a pseudoscalar (blue diamonds), and two mixed states corresponding to $f_{g i}=0.5$ with $\phi_{g i}=0$ (green squares) and $\phi_{g i}=\pi / 2$ (closed magenta circles) are shown. For the left plot, $i=4$. For the right plot, $i=2$. Black crosses show the distribution of the mixed states with no interference. . . . . . . . . . . . . .

4.1 Distribution of $m_{j j}$ (top left), TCHE b-tagging discriminant (top right), and MET significance, $2 \ln \lambda\left(E_{T}^{\text {miss }}\right.$, (bottom left). Event category populations are shown in the bottom right plot. Filled histograms represent expectation of background events. Open, red histograms representation the expectation of a $400 \mathrm{GeV}$ Higgs boson whose cross section has been enhanced by $100 \times$. All events satisfy the preselection requirements. . . . . . . . . . . . . . . . . .

4.2 Empirical derivation of 5D PDF for $\mathrm{Z}+$ jets events. Points represent expected distributions of events between $475<m_{Z Z}<550 \mathrm{GeV}$ from MC simulation, lines represent the final model at the median $m_{Z Z}$ value. 62

4.3 Empirical derivation of 5D PDF for signal events. Points represent expected distributions of events for $m_{H}=500 \mathrm{GeV}$ from MC simulations, lines represent the final model at the median $m_{Z Z}$ value. . . . . . . . 


\section{LIST OF FIGURES}

4.4 Distribution of $m_{Z Z}$ after optimal cut on angular D (right) and traditional variables, (left). Maroon histogram represents expected distribution of a $400 \mathrm{GeV}$ SM Higgs, blue and green histograms represent different SM backgrounds from MC simulations. . . . . . . . . . .

4.5 Distribution of 5 angles used to build the angular likelihood discriminant, shown in the bottom right plot. Filled histograms represent expectation of background events. Open, red histograms representation the expectation of a $400 \mathrm{GeV}$ Higgs boson whose cross section has been enhanced by $100 \times$. All events satisfy the preselection requirements. .

4.6 The $m_{Z Z}$ invariant mass distribution after final selection in three categories: 0 b-tag (top), 1 b-tag (middle), and 2 b-tag (bottom). The low-mass range, $120<m_{Z Z}<170 \mathrm{GeV}$ is shown on the left and the high-mass range, $183<m_{Z Z}<800 \mathrm{GeV}$ is shown on the right. Points with error bars show distributions of data and solid curved lines show the prediction of background from the control region extrapolation procedure. In the low-mass range, the background is estimated from the $m_{Z Z}$ for each Higgs mass hypothesis and the average expectation is shown. Solid histograms depicting the background expectation from simulated events for the different components are shown. Also shown is the SM Higgs boson signal with the mass of 150 (400) GeV and cross section 5 (2) times that of the SM Higgs boson, which roughly corresponds to the expected exclusion limits in each category. . . . .

4.7 Signal shapes models for $400 \mathrm{GeV}$ (top row) and $130 \mathrm{GeV}$ (bottom row) signals for each of the three b-tag categories, 0 b-tag (left), 1 b-tag (middle), and 2 b-tag (right). . . . . . . . . . . . . .

4.8 Signal efficiency parametrization in each of the 6 different categories of the high mass signal samples. . . . . . . . . . . . . . . . . . .

4.9 Signal efficiency parametrization in each of the 6 different categories of the low mass signal samples. . . . . . . . . . . . . . . . . . .

4.10 Observed (solid) and expected (dashed) 95\% CL upper limit on the ration $f$ the production cross section o the SM expectation for the Higgs boson obtained using the $C L_{s}$ technique. The $68 \%(1 \sigma)$ and $95 \%(1 \sigma)$ ranges of expectation for the background-only model are shown with green and yellow bands, respectively. The solid line at 1 indicates the SM expectation. Left: low-mass range, right: high-mass range. . . . 4.11 Invariant mass distribution of the $4 \ell$ system for events between $70<$ $m_{4 \ell}<1000 \mathrm{GeV}$ (left) and between $100<m_{4 \ell}<180 \mathrm{GeV}$ (right). All final states have been included. Points with error bars represent a sum of the $\sqrt{s}=7 \mathrm{TeV}$ and $\sqrt{s}=8 \mathrm{TeV}$ datasets. Solid histograms represent background estimations. The open red histogram represents simulation of a SM Higgs, $m_{H}=126 \mathrm{GeV}$. . . . . . . . . . . 


\section{LIST OF FIGURES}

4.12 Distribution of $m_{4 \ell}$ and $K_{D}$ in various regions. Contours in the left and right plot represent the background expectation of continuum ZZ events. Contours in the middle plot represent signal plus background expectation, where signal is a SM Higgs, $m_{H}=126 \mathrm{GeV}$. Points with error bars represent the individual events observed in the four different final states. Horizontal error bars represent the reconstructed mass uncertainties. . . . . . . . . . . . . . . .

4.13 Distribution of $p_{T, 4 \ell}$ in the non-dijet category (top row) and $\mathscr{D}_{\text {jet }}$ in the dijet category (bottom row) for expectation of a VBF produced (left column) or a gluon-gluon fusion produced Higgs boson with $m_{H}=$ $126 \mathrm{GeV}$. Points with error bar show the distribution of observed $4 \mu$ (circles), $4 e$ (triangles), and $2 e 2 \mu$ (squares) events. . . . . . . . .

4.14 Expected and observed 95\% confidence level upper limit on $\sigma / \sigma_{S M}$ as a function of the hypothetical Higgs mass, $m_{H}$, in the range [110-1000]. The green and yellow bands represent the one and two sigma bands of the expected distribution, respectively. . . . . . . . . . . . .

4.15 Expected and observed p-value with respect to the background only hypothesis as a function of the hypothetical Higgs mass, $m_{H}$, in the range [110-180] (left) and [110-1000] (right). Solid lines show the observed p-values while dashed lines show the expected p-values, assuming a SM Higgs. Green lines show p-values obtained using only the information about $m_{4 \ell}$ distributions. Red lines show p-values obtained using $m_{4 \ell}$ vs $K_{D}$ distributions. . . . . . . . . . . . . . . . . . .

4.16 Best fit signal strength modifier, $\mu$, is both the dijet and untagged categories as well the combination of all channels (black line). Red bar represent the $68 \%$ confidence intervals for each of the individual measurements. The green band represents the $68 \%$ confidence interval for the combined measurement.

4.17 Distributions of $\mathscr{D}_{b k g}$ (left) and $\mathscr{D}_{b k g}$ (right). Expected distribution for a $125.6 \mathrm{GeV}$ SM Higgs boson is shown in red, the continuum ZZ background in blue, and the reducible background in green. . . . . . . 4.18 Distributions of $\mathscr{D}_{J^{P}}$ for $J^{P}=0^{-}, 0_{h}^{+}$, and $1^{-}$(first row), $J^{P}=1^{+}$, $2_{m}^{+}(g g)$, and $2_{m}^{+}(q \bar{q})$ (second row), $J^{P}=2_{h}^{+}, 2_{h}^{-}$, and $2_{b}^{+}$(third row), and production independent tests of $J^{P}=1^{-}, 1^{+}$, and $2_{m}^{+}$(fourth row). Expected shapes for a 125.6 GeV SM Higgs boson is shown in red, the continuum background in blue, the reducible background in green, and observed data in the point with error bars. . . . . . . . . . . . 


\section{LIST OF FIGURES}

4.19 Distribution of expected and observed test statistics for various hypothesis test. Orange histograms represent toys generated under the null hypothesis, SM background plus a SM Higgs boson. Blue histograms represent toys generated under the alternative hypothesis. The red arrow shows the value of the observed test statistic. All resonances are assumed to have a mass of $125.6 \mathrm{GeV}$. . . . . . . . . . . . . . .

4.20 Distribution of $-2 \ln \mathscr{L}$ versus $\left(\mu, f_{a 3}\right)$, Blue and teal band represent the $68 \%$ and $95 \%$ confidence level contours, respectively. The point represents the location of the maximum likelihood. . . . . . . . . .

4.21 Distribution of $-2 \ln \mathscr{L}$ versus $f_{a 3}$. The black line in the right plots represents the expected distribution calculated from fitting the Asimov dataset; the blue line represents the observed distribution. The signal strength, $\mu$, has been profiled. . . . . . . . . . . .

5.1 Distributions of $D_{C P}$ (right) and $D_{\text {int }}$ (left) are shown for several scalar models. Distributions for a SM Higgs are respresented by red circles, pure alternative scalar models (either $0^{-}$or $0_{h}^{+}$) by blue diamonds, and mixed scalar models corresponding to $f_{a 3}=0.5$ and $f_{a 2}=0.5$ $\left(\phi_{a i}=0\right)$ for left and right plots, respectively by green squares. The closed magenta circles in the right plot corresponds to a mixed scalar

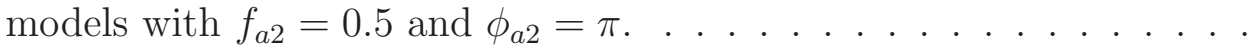

5.2 Distribution of best-fit $f_{a 3}$ values from a large number of generated experiments using either the $1 \mathrm{D}$ fit of the $D_{0^{-}}$distributions (solid black), 7D fits with only $f_{a 3}$ unconstrained (dashed magenta), or 7D fits with $f_{a 3}$ and $\phi_{a 3}$ unconstrained (dotted blue). . . . . . . . . . . .

5.3 Distributions of masses (top row), production angles (middle row), and helicity angles (bottom row), in the $H \rightarrow Z Z^{*} \rightarrow 4 \ell$ analysis at the LHC. Open red points show simulated events for the SM Higgs boson with curves showing projections of analytical distributions. Solid black points show background distributions with curves showing projections of analytical parametrization. Distributions before (circles) and after (squares) detector effects are shown. . . . . . . . . . . .

5.4 Distributions of fitted values of $f_{a 3}$ from a large number of generated experiments in the $H \rightarrow Z Z^{*} \rightarrow 4 \ell$ channel at the LHC. Results for the $300 \mathrm{fb}^{-1}$ (dotted) and $3000 \mathrm{fb}^{-1}$ (solid) scenarios are shown. . . .

5.5 Diagrams showing the different processes produced via the HZZ amplitude. The $e^{+} e^{-} \rightarrow Z^{*} \rightarrow Z H \rightarrow 2 \ell 2 b$ process in the $Z^{*}$ and $\mathrm{H}$ rest frame are shown in the left and middle plot, respectively. The $p p \rightarrow H \rightarrow Z Z^{*} \rightarrow 4 \ell$ process is shown in the $\mathrm{H}$ rest frame is shown in the right plot. . . . . . . . . . . . . . . 


\section{LIST OF FIGURES}

5.6 Angular distributions, $\cos \theta_{1}$ (left), $\cos \theta_{2}$ (middle), and $\Phi$ (right), of four different scalar models of the process $e^{+} e^{-} \rightarrow Z^{*} \rightarrow Z H$. Markers show angular distributions from simulations while lines show projections of the angular distributions presented in Section 3. Red line/circles represent a SM Higgs, blue lines/diamonds represent a pseudoscalar, green lines/squares and purple lines/solid circles represent a mixed parity scalar $\left(f_{a 3}=0.1\right)$ with various phases. . . . . . . . . . .

5.7 Expected distribution of three helicity angles for a SM Higgs boson (red) and the SM background (black) before (solid lines) and after (dashed lines) acceptance cuts. . . . . . . . . . . . . . .

5.8 Distribution of the best-fit value of $f_{a 3}$ from a large number of generated experiments. Toys were generated using a value of $f_{a 3}=0.1$. . 117

6.1 Distribution of test statistics for SM Higgs toys (blue), alternative $J^{P}$ signals toys (orange), and the observed test statistic (points). . . . . .

6.2 Distributions of the test statistic comparing the SM Higgs hypothesis against the $J^{P}=2_{m}^{+}$hypothesis using a simultaneous fit of the signal strength in the ZZ and WW channels. The orange distribution represents the SM Higgs toys, the blue distribution represents the $2_{m}^{+}$ hypothesis. The red arrow shows the observed test statistic. . . . . .

6.3 Best-fit signal strength modifier, $\mu$, for various production and decay modes. Red error bars represent the $68 \%$ confidence interval of the individual measurements. Black lines represent the combined measurement of all channels (production and decay); the green band represents the the $68 \%$ confidence interval. All fits are done for a fixed mass hypothesis, $m_{H}=125.7 \mathrm{GeV}$, which correspond to the combined best-fit value. . . . . . . . . . . . . . . . . . .

6.4 Summary of the fits for deviations in the coupling for the generic fiveparameter model not including effective loop couplings, expressed as function of the particle mass. For the fermions, the values of the fitted Yukawa couplings hff are shown, while for vector bosons the squareroot of the coupling for the hVV vertex divided by twice the vacuum expectation value of the Higgs boson field. Particle masses for leptons and weak boson, and the vacuum expectation value of the Higgs boson are taken from the PDG. For the top quark the same mass used in theoretical calculations is used $(172.5 \mathrm{GeV})$ and for the bottom quark the running mass $m_{b}\left(m_{H}=125.7 \mathrm{GeV}\right)=2.763 \mathrm{GeV}$ is used. . . . . 


\section{Chapter 1}

\section{Introduction}

The Standard Model (SM) of particle physics is a mathematical description of the fundamental particles and their interactions. Within the SM, particles are described by quantized excitations of spin- 0 , spin- $1 / 2$, and spin- 1 fields which are solutions to the Klein-Gordon, Dirac, and Proca equations, respectively. These equations govern the time evolution of each field. Other spin states can arise from bound states.

The interactions of fields are encoded in the SM Lagrangian. For example, the electromagnetic interactions of electrons are described by the Lagrangian

$$
\mathscr{L}_{E M}=\bar{\psi}\left(i \gamma^{\mu}\left(\partial_{\mu}+i e A_{\mu}\right)-m\right) \psi-\frac{1}{4} F_{\mu \nu} F^{\mu \nu} .
$$

The probability for some initial state evolving into some final state can be be expanded in powers of the coupling constant, $e$, according to the modulus squared matrix 


\section{CHAPTER 1. INTRODUCTION}

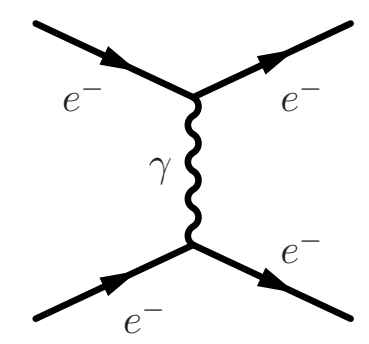

Figure 1.1: Feynman diagram depicting electron-electron scattering via the electromagnetic interaction.

element

$$
\left|<\psi_{e} \psi_{e}\right| e^{\bar{\psi}_{e}\left(i \gamma^{\mu}\left(\partial_{\mu}+i e A_{\mu}\right)\right) \psi_{e}}\left|\psi_{e} \psi_{e}>\right|^{2} .
$$

Often, the amplitude of a process at some order in the couplings is represented by a Feynman diagram such as the one in Figure 1.1 which represents electron-electron scattering to lowest order in a purely electromagnetic theory.

The interactions of the SM are derived by enforcing local gauge symmetries and thus can be described through a symmetry group. For example, the electromagnetic interactions are known to be generated from a U(1) gauge symmetry. Each symmetry has a corresponding charge which is conserved and which the gauge mediators couple to. For example, the photon couples to the electric charge, e. Thus, specifying the gauge symmetries and the charges of particles provides a clear description of particle interactions.

Currently, the SM describes three of the four known forces: the electromagnetic, the weak, and the strong force, which are generated from $\mathrm{U}(1), \mathrm{SU}(2)$, and $\mathrm{SU}(3)$ gauge symmetries, respectively. The charges of the fundamental fields known to exist 


\section{CHAPTER 1. INTRODUCTION}

\begin{tabular}{l|c|c|c}
\hline \hline particle & $\mathrm{Q}$ & $T_{3}$ & colored \\
\hline \hline$e_{L}, \mu_{L}, \tau_{L}$ & -1 & $-1 / 2$ & no \\
$e_{R}, \mu_{R}, \tau_{R}$ & -1 & 0 & no \\
$\nu_{L}$ & 0 & $1 / 2$ & no \\
$u_{L}, c_{L}, b_{L}$ & $2 / 3$ & $1 / 2$ & yes \\
$u_{R}, c_{R}, b_{R}$ & $2 / 3$ & 0 & yes \\
$d_{L}, s_{L}, t_{L}$ & $-1 / 3$ & $-1 / 2$ & yes \\
$d_{R}, s_{R}, t_{R}$ & $-1 / 3$ & 0 & yes \\
\hline
\end{tabular}

Table 1.1: List of SM particles and their charges. Q represents the charge of the $S U(1)_{\text {em }}$ gauge symmetry, $T_{3}$ the broken $\mathrm{SU}(2)$ gauge symmetry, and color the charge of the $S U(3)$ gauge symmetry.

in the SM are shown in Table 1.1. The photon only couples to electrically charged particles $(\mathrm{Q})$, the $\mathrm{W}$ boson couples to particles charged under weak isospin $\left(T_{3}\right)$, and the gluons couple to colored particles. As the names suggest, at low energies, the strong force is the strongest and the weak force is the weakest. It is commonly believed that these interactions should all be unified at some energy scale where the strength will become comparable.

Naively, the idea of interactions arising from enforcing gauge symmetries produces inconsistencies between theory and experiments. Even at the time when the $\mathrm{SU}(2)$ structure of the weak interactions was first proposed by Glashow [2], the W boson was known to be massive. However, mass terms in a Lagrangian break gauge invariance. This internal inconsistency suggested that the SU(2) gauge symmetry must be broken in a specific way in order to allow the weak vector bosons to be massive, a process known as electroweak symmetry breaking. 
CHAPTER 1. INTRODUCTION

\subsection{Electroweak Symmetry Breaking}

In 1963, Phil Anderson proposed that spontaneously broken symmetries could provide a theoretical framework for explaining massive gauge bosons in non-relativistic systems [3]. In 1964, these ideas were studied in the context of relativistic quantum field theories. It was shown that a complex scalar field whose potential was particularly chosen could spontaneously break a gauge symmetry and generate gauge boson masses through the interaction of this field with the gauge bosons [4-7]. Most notably, Peter Higgs suggested that this would also predict the presence of a new massive scalar particle [6].

Glashow, Weinberg, and Salam $[2,8,9]$ showed that the Higgs mechanism could be used to break a $S U(2) \times U(1)_{Y}$ symmetry to a $U(1)_{e m}$ symmetry producing all of the known electroweak interactions and massive weak gauge bosons. The GlashowWeinberg-Salam (GWS) model predicted a massive, neutral gauge boson, the Z boson, whose mass would be around $90 \mathrm{GeV}$; this was confirmed indirectly through electronneutrino scattering [10-12]. The Z boson was later directly detected $[13,14]$. Another experimental signature of the GWS model was that there should exist a chargeless, colorless, spinless, massive boson, similar to that suggested by Higgs; this particle is now commonly referred to as the Higgs boson. Except for its mass all properties of this particle could be calculated whithin the framework of the SM (see Section 3).

Electroweak symmetry breaking is the cornerstone of the SM model and illuminating the exact mechanism by which it occurs is paramount to our understanding of the 


\section{CHAPTER 1. INTRODUCTION}

universe. Thus, the experimental verification of the Higgs boson and its properties has been the top priority of the field of particle physics for nearly fifty years.

\subsection{Higgs Boson Constraints}

Several accelerators have been built to discover the Higgs boson, the first of which was the Large Electron-Positron (LEP) collider which accelerated electrons and positrons to energies up to $209 \mathrm{GeV}$. Although a broad range of Higgs boson masses were accessible to LEP experiments, no evidence was found and 95\% confidence level exclusion limits were set for all masses up to $114.4 \mathrm{GeV}$ [15]. However, high precision measurements made on a number of SM quantities could be used to constrain the Higgs boson mass under the assumption it were to exist according to the SM. These constraints suggested that a SM Higgs boson would be more likely in the range $m_{H} \lesssim 185 \mathrm{GeV}[16]$.

The Tevatron and its experiments also contributed major efforts towards Higgs searches. As a $2 \mathrm{TeV} p \bar{p}$ collider, considerably larger masses were accessible compared to LEP. However, no evidence of the Higgs boson was found and 95\% confidence level exclusion limits were set for Higgs boson mass between $162<m_{H}<166 \mathrm{GeV}$ [17]. Despite the lack of a Higgs boson observation, the discovery of the top quark and measurement of its mass helped to refine calculations of the Higgs boson production cross section and branching ratios which include contributions from virtual top quarks. 


\section{CHAPTER 1. INTRODUCTION}

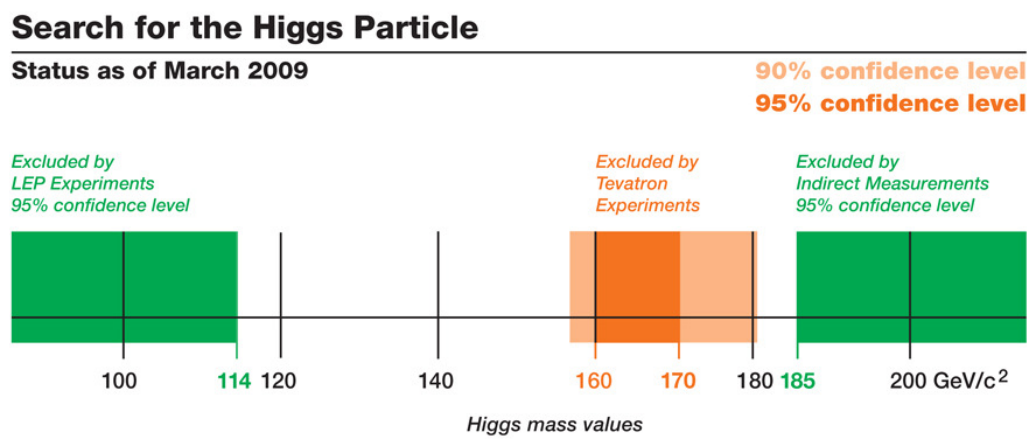

Figure 1.2: Constraints on the SM Higgs boson mass from Tevatron and LEP experiments either through direct searches or indirect evidence based on precision measurements.

By the time the LHC was delivering beams, theory calculations had been refined and both direct limits and indirect limits had been set by LEP and Tevatron experiments. Figure 1.2 summarizes the status of Higgs searches at this time. Since the Higgs mechanism must unitarize VV scattering, there is a limited mass range for which the Higgs mechanism makes sense, $m_{H} \lesssim 1000 \mathrm{GeV}$. This theoretical upper bound and the experimental lower bound from the LEP direct search limits suggest that the LHC would suffice to make the final statement about the existence of the Higgs boson, nearly 50 years after it was first proposed.

\subsection{Beyond the SM Higgs}

The Higgs mechanism, as described in the SM, conveniently solved several problems: the existence of massive gauge bosons, the apparent disparity between the electromagentic and weak forces, and the non-unitarity of longitudinal weak boson 


\section{CHAPTER 1. INTRODUCTION}

scattering. Yet, despite its success at describing terrestrial experiments, the SM fails to explain a number of phenomena observed in the universe.

It is thought that more than $95 \%$ of the known universe consists of dark matter $(\sim 27 \%)$ and dark energy $(\sim 68 \%)$ [18]. Since there is currently no way to explain either dark matter or dark energy within the SM, the SM can only attempt to explain about $5 \%$ of the energy of the universe.

The overabundance of matter, as opposed to anti-matter, in the universe, is a phenomenon known as the baryon asymmetry. It was shown by Sakharov [19] that there are three necessary conditions a model of baryogenesis must satisfy: baryon-number violation, charge-symmetry and charge-parity-symmetry violation (CP-violation), and interactions which are out of thermal equilibrium at early stages of the universe. Although it has been shown that the SM does contain the three necessary conditions for baryogenesis, it is believed to be insufficient for explaining the degree of baryonic asymmetry in the visible universe $[20,21]$. As such, additional sources of CP-violation in the SM would provide a promising solution to the baryon-asymmetry problem.

The expected naturalness of electroweak symmetry breaking is also often cited as evidence for physics beyond the SM. Quantum corrections to the Higgs boson mass have been found to be much larger than the physical Higgs boson mass [22]. If it is to provide the necessary cancellations to preserve unitarity in longitudinal weak boson scattering, these corrections should be offset by the bare Higgs boson mass in order to keep the physical mass small. This introduces what is known as fine tuning. The 


\section{CHAPTER 1. INTRODUCTION}

unnaturalness of the Higgs boson mass relative to the Plank scale $\left(10^{19} \mathrm{GeV}\right)$ is also known as the hierarchy problem.

There are a number of proposed solutions to the fine tuning problem, some of which could also provide solutions to some of the problems noted above, for example, Supersymmetry (SUSY). Since SUSY predicts that all fermions have a symmetry with a corresponding boson, all Feynman diagrams which provide quantum corrections to the Higgs boson mass have a canceling partner which removes the large quantum corrections $^{1}$. SUSY is also thought to provide a natural dark matter candidate and is a prerequisite for string theory, which naturally incorporates gravity. Finally, it is possible for SUSY to allow for additional CP-violation in the Higgs sector. Recent work has studied this idea in the more generic framework of type-II 2 Higgs doublet models (2HDM) and found that the amount of additional CP-violation possible in the Higgs sector could provide a reasonable model for baryogenesis [26].

Other explanations of fine tuning include composite Higgs models or RandallSundrum models of gravity. Composite Higgs models interpret the Higgs mechanism as only an effective theory and introduce a new strongly interacting QCD-like force above the electroweak scale. It was shown by Randall and Sundrum [27] that higherdimensional models with warped space-time metrics can provide a natural explaination of the hierarchy problem and thus fine tuning.

\footnotetext{
${ }^{1}$ Although this was not the original motivation for SUSY, it was later suggested to provide a solution to fine-tuning in the SM by Witten [23], Veltman [24], and Kaul [25]. This is discussed in more detail elsewhere [22].
} 


\section{CHAPTER 1. INTRODUCTION}

\subsection{Summary}

Although many of the above arguments for naturalness in the SM are heuristic, they suggest that the Higgs sector could be a window to physics beyond the SM through: the discovery of multiple scalars, the discovery of CP-violation in Higgs interactions, or the discovery of Higgs compositeness. Today, the muon magnetic moment has been calculated and measured to an extremely high precision and has been used as a test of the SM as well as a probe for new physics. Analogously, the Higgs boson may become the next source of high precision tests of the SM which may ultimately illuminate the existence of new physics.

This thesis will discuss several analyses designed to search for a SM Higgs boson using tools which have been developed to not only provide increased sensitivity to signal events but also to measure properties of observed resonances. Chapter 2 will discuss the experimental details of the Large Hadron Collider (LHC) and the Compact Muon Solenoid (CMS). Chapter 3 will discuss Higgs phenomenology at the LHC. Chapter 4 will present two analyses designed to search for the SM Higgs boson using the $Z Z \rightarrow 2 \ell 2 q$ signature and using the $Z Z \rightarrow 4 \ell$ signature. The latter will include the discovery and characterization of a new bosonic resonance using the tools developed in Chapter 3. Chapter 5 will discuss the prospects of precision measurements of Higgs boson properties at both the LHC and a future $e^{+} e^{-}$collider. Finally, Chapter 6 will discuss the interpretation of these results in the context of the beyond the SM physics mentioned above. 


\section{Chapter 2}

\section{Experimental Setup}

\subsection{The Large Hadron Collider}

The Large Hadron Collider was designed to accelerate two beams of protons up to energies of $7 \mathrm{TeV}$ using a $27 \mathrm{~km}$ storage ring and 1232 individual $8.33 \mathrm{~T}$ dipole magnets. Although it is also capable of accelerating heavier nuclei up to energies of $2.76 \mathrm{TeV}$, heavy ion physics is outside the scope of this work. The proton energies accessible to the LHC are a factor of seven times higher than its most advanced predecessor, the Tevatron. These energies are not only important for accessing new particles which might exist at large invariant mass, on the order of several $\mathrm{TeV}$, they are also necessary for efficient production of moderately heavy particles, like the Higgs boson or the top quark. For a $125 \mathrm{GeV}$ Higgs boson these energies provide a factor of $\sim 50$ in total cross section over the production cross section at the Tevatron. 


\section{CHAPTER 2. EXPERIMENTAL SETUP}

\begin{tabular}{l|c|c}
\hline \hline Energy per nucleon & $E$ & $7 \mathrm{TeV}$ \\
Dipole field at $7 \mathrm{TeV}$ & $B$ & $8.33 \mathrm{~T}$ \\
Design luminosity & $\mathscr{L}$ & $10^{34} \mathrm{~cm}^{-2} \mathrm{~s}^{-1}$ \\
Bunch separation & & $25 \mathrm{~ns}$ \\
No. of bunches & $k_{B}$ & 2808 \\
No. of particles/bunch & $N_{p}$ & $1.15 \times 10^{11}$ \\
\hline Collisions & $\beta^{*}$ & $0.55 \mathrm{~m}$ \\
\hline$\beta$-value at IP & $\sigma^{*}$ & $16.7 \mu \mathrm{m}$ \\
RMS beam radius at IP & $\tau_{L}$ & $15 \mathrm{hr}$ \\
Luminosity lifetime & $\equiv 20$ \\
Number of collisions/crossing & $n_{c}$ & \\
\hline \hline
\end{tabular}

Table 2.1: Relevant operational LHC parameters and there values at under design conditions.

The LHC has the capability to collide bunches of $1 \times 10^{11}$ protons every $25 \mathrm{~ns}$ at $\beta^{*}=.55$ and $\sigma^{*}=16.7$. These parameters and others, summarized in Table 2.1, combine to allow the LHC to produce instantaneous luminosities of up to $10^{34} \mathrm{~cm}^{-2} \mathrm{~s}^{-1}$ according to

$$
\mathscr{L}=\frac{\gamma f k_{B} N_{p}^{2}}{4 \pi \epsilon_{n} \beta^{*}} F
$$

where $\gamma$ is the Lorentz factor, $f$ is the revolution frequency, $k_{B}$ is the number of protons per bunch, $\epsilon_{n}$ is the betatron function at the interaction point, and $F$ is the reduction factor due to the crossing angle. This translates to roughly 1 billion proton-proton interactions per second and up to 50 collisions per bunch crossing.

These conditions provide the necessary environment to probe the SM and discover new particles, but also an extreme environment for reconstructing particle paths and energy deposits with a high degree of accuracy and efficiency. The inclusive 


\section{CHAPTER 2. EXPERIMENTAL SETUP}

proton-proton cross section at $14 \mathrm{TeV}$ is approximately $100 \mathrm{mb}$, roughly 10 orders of magnitude larger than the largest Higgs cross sections. At design luminosity, this corresponds to an event rate of $10^{9} \mathrm{~Hz}$. The large number of proton-proton collisions produce a considerable amount of background noise which can produce extra particles from secondary interactions, also known as pileup, as well an overall increase in the energy deposited in the calorimeters. The high rate of collisions at the LHC far exceeds the capabilities of the CMS Data Acquisition (DAQ) system. As a result it is necessary to use fast hardware logic to filter the vast majority of events. The short time between bunch crossings also puts significant constraints on detector design since sub-detectors should have fast response times and low occupancy. High granularity tracking will be necessary for high precision vertexing in order to mitigate the effects of pileup.

\subsection{The Compact Muon Solenoid}

The Compact Muon Solenoid (CMS), is a general purpose particle detector. It was designed to not only have a broad scope of discovery potential but also to mitigate the extreme conditions created by the LHC. CMS is made up of several different types of apparatuses designed to improve identification of particles and measure their properties. There is a two-stage trigger system to filter the extreme rates coming from the LHC. There is an all silicon tracking system at the center to carefully record 


\section{CHAPTER 2. EXPERIMENTAL SETUP}

the positions of charged particles passing through the detector. There is a 4 Tesla magnet to bend charged particles providing the tracker and muon system sensitivity to the momentum of charged particles. There are two calorimeters designed to induce particle showers which can then be used to measure energy deposits. Finally, there is a Muon system at the edge of the detector to detect semi-stable, charged particles with long interaction lengths, e.g. the muon. This chapter provides a brief description of these sub-detector.

\subsubsection{Magnet}

CMS employs a $4 \mathrm{~T}$ superconducting aluminum solenoid magnet to bend tracks for both charge identification and momentum resolution. The field strength was chosen to have good momentum resolution, $\Delta p / p \equiv 10 \%$ at $p=1 \mathrm{TeV} / \mathrm{c}$. The magnet has an inner bore of $5.9 \mathrm{~m}$, large enough to house the tracker and both calorimeters, and a length of $12.9 \mathrm{~m}$. Drawing a current of $19.5 \mathrm{kA}$, the magnet's total stored energy is $2.7 \mathrm{GJ}$, making it one of the largest magnets in the world. The outer return yolk of the magnet concentrates the magnetic field in the region near the muon system, which is placed outside of the solenoid. 


\section{CHAPTER 2. EXPERIMENTAL SETUP}

\subsubsection{Trigger and data acquisition}

The event rate delivered to CMS is approximately $10^{9} \mathrm{~Hz}$. However, only about $1000 \mathrm{~Hz}$ can be processed by CMS. This requires a large, yet efficient, rejection scheme. CMS employs a two level system to make fast decisions on which events to record. The level-1 system consists of custom electronics which monitor the activity in the calorimeters and the muon system. Decisions are based on raw energy and momentum thresholds. The level-1 system reduces the event rate down to roughly $100 \mathrm{kHz}$ while the High-Level Trigger (HLT), an on-line processing farm which executes reconstruction software, further reduces the rate to $1000 \mathrm{~Hz}$. Customized HLT selections are designed to ensure high efficiencies for different physics signatures.

\subsubsection{Electromagnetic Calorimeter}

The Electromagnetic Calorimeter (ECal) is a high granularity calorimeter intended to induce electromagnetic showers which are collected by crystals and either avalanche photodiodes (barrel) or vacuum phototriodes (endcap). The material used is scintillating lead tungstate crystal which was chosen for its: short radiation length $\left(X_{0}=0.89 \mathrm{~cm}\right)$ and Moliere length $(2.2 \mathrm{~cm})$; the time scale in which showers occur ( $80 \%$ of light is emitted in $25 \mathrm{~ns}$ ); and the radiation hardness. The ECal is divided into barrel (EB) and endcap (EE) regions.

The EB region has an inner radius of $129 \mathrm{~cm}$ and is constructed from 36 iden- 


\section{CHAPTER 2. EXPERIMENTAL SETUP}

tical supermodules, each covering half of the barrel in the z-direction (1.479 unit of pseudorapidity). Each individual crystal covers 1 degree in both $\Delta \phi$ and $\Delta \eta$, corresponding to a cross sectional area of $22 \times 22 \mathrm{~mm}^{2}$, and is $230 \mathrm{~mm}$ long, corresponding to $25.8 X_{0}$.

The EE region is located at a distance of $314 \mathrm{~cm}$ along the z-direction and covers the pseudorapidity range $1.479<|\eta|<3.0$. The crystals are clustered into $5 \times 5$ supercrystals which are combined to form semi-circular structures. Each crystal has a cross sectional area of $28.6 \times 28.6 \mathrm{~mm}^{2}$ and is $220 \mathrm{~mm}\left(24.7 X_{0}\right)$ in length. The endcap region is also preceded by a preshower which consists of a lead absorber whose thickness is $2-3 X_{0}$ followed by 2 planes of silicon strip detectors.

The energy response of the ECal was measured in test beams. The energy resolution was parameterized according to

$$
\left(\frac{\sigma}{E}\right)^{2}=\left(\frac{S}{\sqrt{E}}\right)^{2}+\left(\frac{N}{E}\right)^{2}+C^{2}
$$

where $\mathrm{S}, \mathrm{N}$ and $\mathrm{C}$ represent the stochastic, noise, and constant contributions.

\subsubsection{Hadronic Calorimeter}

The hadronic calorimeter (HCal) consists of brass absorbers and plastic scintillators in which light is collected from wavelength-shifting fibers. Fiber cables transmit light into hybrid photodiodes. The HCal is separated into four regions: the barrel 


\section{CHAPTER 2. EXPERIMENTAL SETUP}

(HB), the outer (HO), the endcap (HE), and the forward (HF) regions.

The HB is made up of 32 towers which cover the pseudorapidity region $|\eta|<1.4$, totaling 2304 towers with a segmentation of $\Delta \eta \phi=0.087 .087$. There are 15 brass plates, each $5 \mathrm{~cm}$ thick and two steel plates for structural stability. Particles entering the HCal barrel region first impinge upon a scintillating layer that is $9 \mathrm{~mm}$ thick, instead of the typical $3.7 \mathrm{~mm}$ for other scintillating layers. More details of the HB design and test beam performance can be found elsewhere [28, 29].

The HO region contains $10 \mathrm{~mm}$ thick scintillators. Each scintillating tile matches the segmentation pattern of the muon system's Drift tubes. The purpose of the $\mathrm{HO}$ is to catch hadronic showers leaking through the HB region. This makes the effective length of the barrel region $10 X_{0}$ and improves missing transverse energy $E_{T}^{\text {miss }}$ resolution.

The HE region consists of $14 \eta$ towers with 5 degree segmentation in $\phi$ and covers the region between $1.3<|\eta|<3.0$. There are 2304 towers in total. The HF region extends between $3.0<|\eta|<5.0$ and is made from steel absorbers and quartz fibers. The fibers are intended to measure Cherenkov radiation. The HF will mainly be used for detecting very forward jets and real-time luminosity measurements. More details of the design and test beam performance of the $\mathrm{HE}$ and $\mathrm{HF}$ can be found elsewhere $[28,29]$. 


\section{CHAPTER 2. EXPERIMENTAL SETUP}

\subsubsection{Muon System}

The Muon system plays an important role in identifying muons. However, because of the vast distance from the interaction point and the muon chambers, momentum resolution of low energy muons is dominated by energy loss due to multiple scattering in the inner detector. In this region, it is found that the tracker dominates the momentum resolution. However, for muons above $\sim 100 \mathrm{GeV}$, the combination of the tracker and muon systems provides superior energy resolution to either system alone. Thus, the muon system plays a major role in momentum resolution of high momentum muons.

The muon system employs three different gaseous detectors, drift tube (DT) chambers, cathode strip chambers (CSC), and resistive plate chambers (RPC). The DT chambers are used in the barrel region, $|\eta|<1.2$, where the magnetic field is low. The CSC detectors are used in the endcaps, $1.2<|\eta|<2.4$, where both rate and the magnetic field is high. The RPC detectors are used both in barrel and endcaps.

The RPCs are fast response detectors with good timing resolution, although do not provide as precise spatial measurements as the DTs and CSCs. Thus, RPCs provide the necessary input to distinguish which bunch crossing a particle should be identified with, which is critical for triggering. All three sub-systems provide a key element to level-1 triggering.

The DTs are arranged in four layers of wheels made up of 12 segments each covering 30 azimuthal degrees. The outermost layer has 1 extra segment in the top 


\section{CHAPTER 2. EXPERIMENTAL SETUP}

and bottom, totaling 14. Each DT is paired with either one or two RPCs, two on either side in the first two layers and one on the inner most edge in the second two layers. A high- $p_{T}$ track can cross up to 6 RPCs and 4 DTs, providing 44 measurements for track reconstruction.

The CSCs are trapezoidal chambers containing 6 gas gaps, each with corresponding cathode strips running radially and anode wires running azimuthally. Charge from ionized gas is collected on strips and wires. Signals on the wires are fast and can be used for level-1 triggering, while cathodes provide a better measurement of position, on the order of $200 \mu m$.

\subsubsection{Tracker}

The CMS tracker is an all silicon detector that consists of more than 16,588 individual silicon modules. These modules are of two basic varieties, pixels which provide a 2-dimensional measurement of particle positions and strips which provide 1-dimensional measurements of particle positions within the plane of the module. The tracker is the closest sub-detector to the interaction point. As such, it is exposed to the highest radiation flux and must be radiation hard to survive the extreme conditions of the LHC. As such, the design of the tracker barrel has been broken into three distinct regions in order to optimize occupancy against signal-to-noise $(\mathrm{S} / \mathrm{N})$ : the pixel barrel (PXB), the tracker inner barrel (TIB), and the tracker outer barrel (TOB). The latter two regions consist of silicon microstrip detectors. 


\section{CHAPTER 2. EXPERIMENTAL SETUP}

\subsubsection{Pixel Modules}

The pixel modules are exposed to the highest particle flux, roughly $10^{7} \mathrm{~Hz}$ at $r=10 \mathrm{~cm}$. As a result, small pixels, $100 \times 150 \mu \mathrm{m}^{2}$, are used giving an occupancy

of about $10^{-4}$ per pixel per bunch crossing. Three layers make up the pixel barrel at radii $r=4.4,7.3$, and $10.2 \mathrm{~cm}$ consisting of 768 pixel modules in total. There are also two endcap disks on either side of the pixel barrel made of 672 pixel modules arranged in a turbine fashion. The layout of the pixel modules is shown in Figure 2.1. In total, there are 66 million pixels which provide precise hit measurements.

\subsubsection{Strip Modules}

The strip modules are arranged into four regions: inner barrel (TIB), outer barrel (TOB), inner disks (TID), and end caps (TEC).

The TIB is divided into 4 layers which extend out to $|z|<65 \mathrm{~cm}$, consisting of 2724 strip modules. The microstrip sensors on each module have a thickness of $320 \mu \mathrm{m}$ and a pitch of $80-120 \mu m$. The two inner most layers of the TIB have stereo modules offset by an angle of $100 \mathrm{mrad}$, providing 2D measurements. The hit position resolution of these modules ranges from 23-34 $\mu \mathrm{m}$ in $r-\phi$ and $230 \mu \mathrm{m}$ in the z-direction.

The TOB is divided into 6 layers extending out to $|z|<65 \mathrm{~cm}$, consisting of 5208 strip modules. Each microstrip sensor has a thickness of $500 \mu m$ and a pitch ranging from 120-180 $\mu \mathrm{m}$. Since the radii of the strip layers is large, strips can be thicker 


\section{CHAPTER 2. EXPERIMENTAL SETUP}

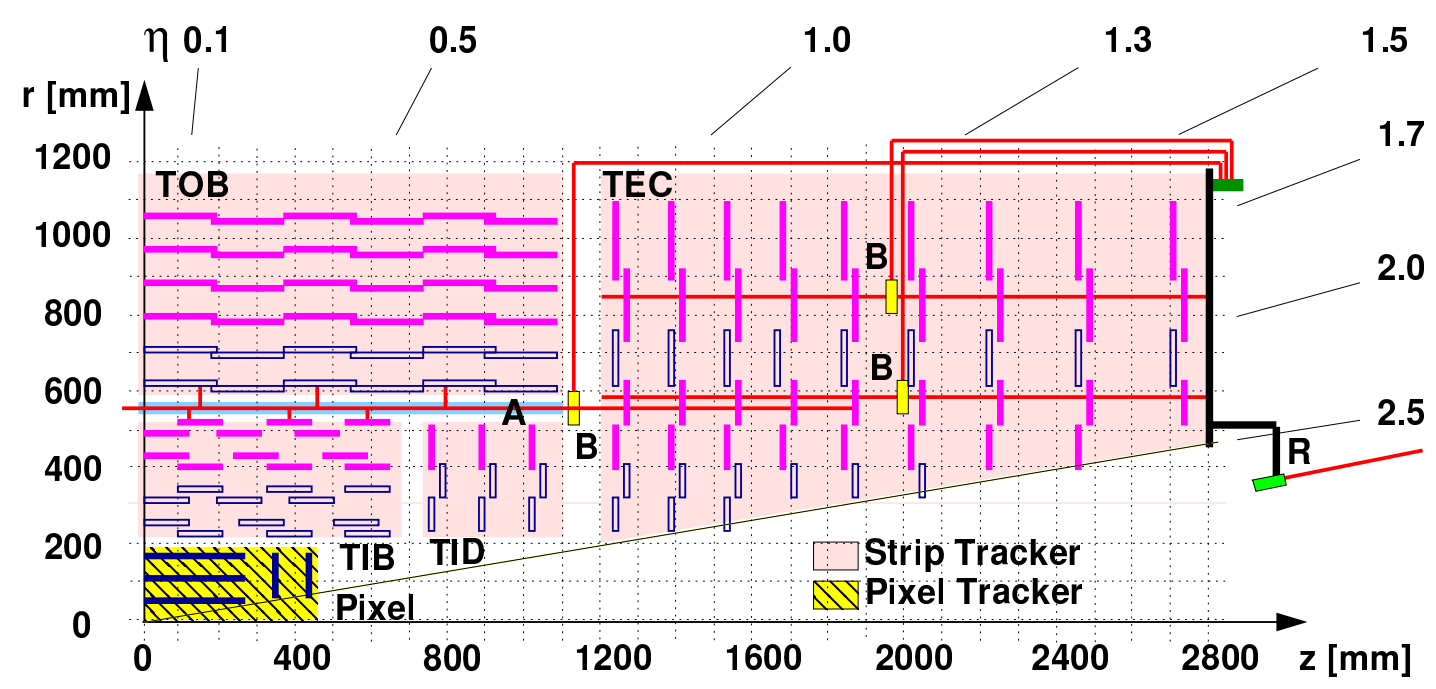

Figure 2.1: Quarter slice of the CMS tracker. Single-sided silicon strip modules are indicated as solid light (purple) lines, double-sided strip modules as open (blue) lines, and pixel modules as solid dark (blue) lines.

in order to have better $\mathrm{S} / \mathrm{N}$ while still have low occupancy. Similar to the TIB, the first two layers of the TOB have stereo modules offset by $100 \mathrm{mrad}$ so that the single point resolution in $r-\phi$ is $35-52 \mu m$ while it is $530 \mu m$ in the z-direction.

The TID is divided into 3 disks, the first two of which are stereo, arranged at various distances between $120<|z|<280 \mathrm{~cm}$. Modules are arranged in wheels around the beam axis. Each microstrip sensor has a thickness of $320 \mu \mathrm{m}$. Similarly, the TEC has 9 disks, the first two and the fifth of which are stereo. The thickness of each microstrip sensor is $500 \mu \mathrm{m}$. 


\section{CHAPTER 2. EXPERIMENTAL SETUP}

\subsubsection{Tracking Performance \& Alignment}

The tracker provides high precision measurements of track parameters for all charged particles; this includes both the momentum and direction of tracks. These track parameters can be used to better understand resonance properties, as will be shown in Chapters 3 and 4 . Thus, the tracker will be one of the most important tools in searching for new resonances, such as the Higgs boson, and understanding their role in nature.

The tracker is also the only detector which can reconstruct vertices, either displaced or not. Vertexing provides critical information to help mitigate the effects of pile-up as well as tagging b-jets. Since pile-up will be a continuing challenge at the LHC, continued performance of the tracker will be critical. The use of the tracker in b-tagging will also play a central role in physics measurements since b-jets provide a distinct signature which is relevant to many models beyond the SM as well of Higgs physics.

In order to ensure high quality performance of track reconstruction algorithms, uncertainties of module positions, which refers to both the location and orientation which are depicted in Figure 2.2, should be reduced to within the precision of each module. For the pixel modules, this precision is around $10 \mu \mathrm{m}$ while for the strips, this precision can be as large as $30 \mu \mathrm{m}$. Because of changing environmental conditions of the detector, the tracker geometry can be time dependent. In order to efficiently determine module positions through run periods, offline track-based alignment algo- 


\section{CHAPTER 2. EXPERIMENTAL SETUP}

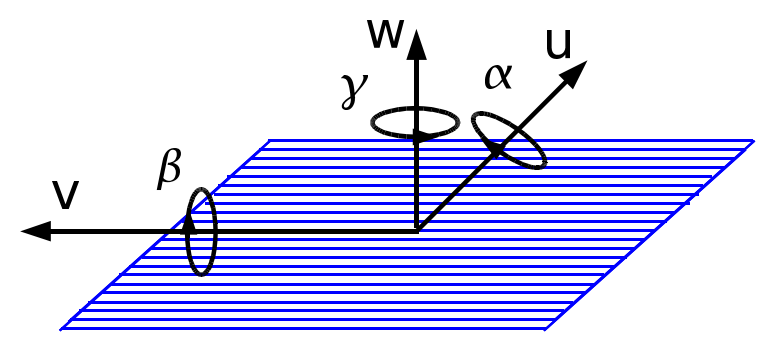

Figure 2.2: Diagram of module position variables, $\mathrm{u}, \mathrm{v}, \mathrm{w}$, and module orientation variables, $\alpha, \beta, \gamma$.

rithms must be employed.

Track-based alignments are intended to determine the position of each module in the tracker from a large collection of reconstructed tracks. Each track is built from a set of charge deposition sites, or hits, on a given module which are used to produce a piece-wise helical trajectory using the Combinatorial Track Finder (CTF) algorithm [30]. Alignment of each module position can be performed by minimizing

$$
\chi^{2}(\vec{p}, \vec{q})=\sum_{j}^{\text {tracks }} \sum_{i}^{\text {hits }} \vec{r}_{i j}^{T}\left(\vec{p}, \vec{q}_{j}\right) V_{i j}^{-1} \vec{r}_{i j}\left(\vec{p}, \vec{q}_{j}\right)
$$

where $\vec{p}$ is the position correction, $\vec{q}_{j}$ is the set of track parameters for the $\mathrm{j}$ tracks, $\vec{r}_{i j}$ are the track residuals, and $\vec{V}_{i j}$ is the covariance matrix. The residuals are defined as $\vec{r}_{i j}=\vec{m}_{i j}-\vec{f}_{i j}\left(\vec{p}, \vec{q}_{j}\right)$, where $\vec{m}_{i j}$ are the measured hit positions and $\vec{f}_{i j}$ are the track trajectory impact point in the plane of the modules. The $\chi^{2}$ function is then minimized with respect to the module position corrections, $\vec{p}$.

Since there are more than 16,588 modules with 6 parameters to be determined, tracker alignment is an extremely difficult problem to solve exactly. As a result, 


\section{CHAPTER 2. EXPERIMENTAL SETUP}

approximations must be employed. One such approximation is to minimize the $\chi^{2}$ for each module individually, ignoring the correlation between the change in parameters between different modules. The correlation is then recovered by recalculating $f_{i j}$ and iterating the procedure many times. Solving for each individual module's position corrections is then reduced to a six-dimensional matrix equation,

$$
\chi^{2}(\vec{p})=\sum_{i}^{\text {hits }} \vec{r}_{i}^{T} \vec{V}_{i}^{-1} \vec{r}_{i}(\vec{p}) .
$$

This local iterative algorithm, described in detail elsewhere $[31,32]$, was employed to produce the first geometry using minimum bias collision tracks.

Validations of tracker geometries are critical to understanding that the output of alignment algorithms improves physics measurements. Several validations which can demonstrate improvements in the tracker geometry are the primary vertex validation and the cosmic splitting validation. Both of these validations provide a direct connection between the tracker geometry and measurements relevant for physics analyses.

The cosmic splitting validation makes use of cosmic tracks recorded during interfills. Cosmic tracks have the unique feature that the tracks can pass through silicon layers on both sides of the tracker. As a result, a cosmic track is qualitatively similar to two collision tracks produced back to back. This feature can be taken advantage of by dividing each cosmic track into subsets of hits and reconstructing these hits into split tracks which are reconstructed independently. The track parameters of the split 


\section{CHAPTER 2. EXPERIMENTAL SETUP}

tracks should, by construction, have the same track parameters. Thus, by comparing the track parameters, resolution and biases can be gauged.

The resolution of individual track parameters can be quantified and compared between different tracker geometries. This is represented by the distribution of the difference of a given track parameters between the two split tracks. This difference can also be compared in slices of other track parameters in order to quantify systematic misalignments.

To demonstrate this, the difference of 5 track parameters: $\Delta d_{x y}, \Delta d_{z}, \Delta \eta, \Delta \phi$, and $\Delta p_{T}$ are shown in Figure 2.3 using cosmic tracks recorded during 2012 Run A. Three geometries are compared, the ideal geometry, the prompt geometry (before alignment) and the Re-RECO geometry (after alignment). Improvements are found over the prompt geometry and in some cases, the aligned geometry is found to be consistent with the ideal geometry tested on MC simulations.

From Figures 2.3, we can see that the average errors of the impact parameters are $25 \mu m(42 \mu m)$ for the transverse (longitudinal) directions with respect to the beam line. The angular variables are found to have extremely good precision, on the level of the $3.2 \times 10^{-4}$ radians for the azimuthal angle, $\phi$, and $\left(4.6 \times 10^{-4}\right)$ for pseudorapidity, $\eta$. The transverse momentum, $p_{T}$, has a relative precision of $1 \%$.

Since the $p_{T}$ distribution of cosmic tracks is dominated by low $p_{T}$ tracks, the $p_{T}$ resolution for high momentum tracks can be better understood by plotting the width of the $\Delta p_{T}$ distribution in bins of $p_{T}$. This is shown in the right plot of Figure 2.4. The 


\section{CHAPTER 2. EXPERIMENTAL SETUP}
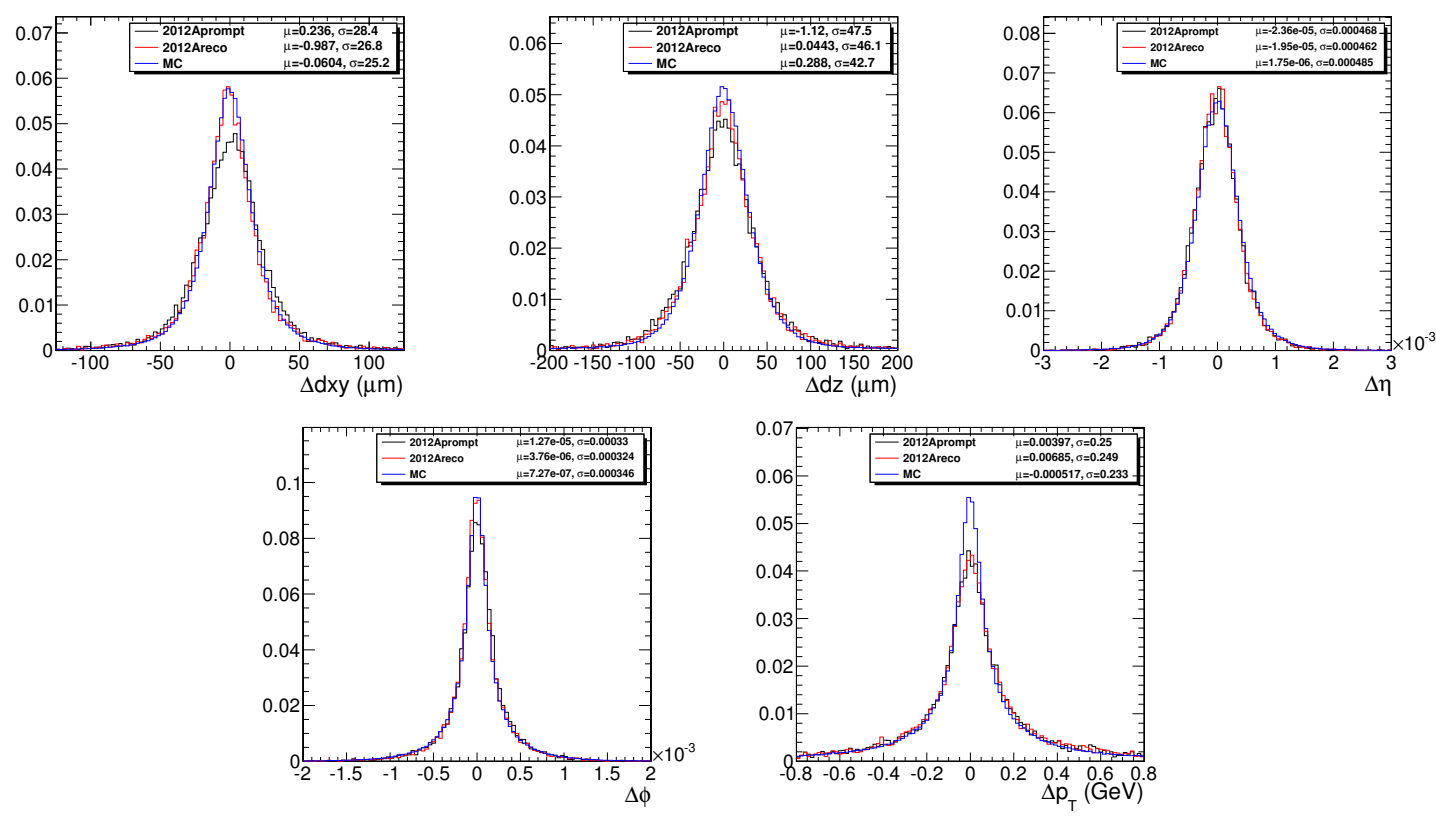

Figure 2.3: Resolution of 5 track parameters from track splitting validation using three geometries, ideal (blue), prompt geometry (black), and the aligned geometry (red). Cosmic track recording during the 2012 Run A period were used. 


\section{CHAPTER 2. EXPERIMENTAL SETUP}
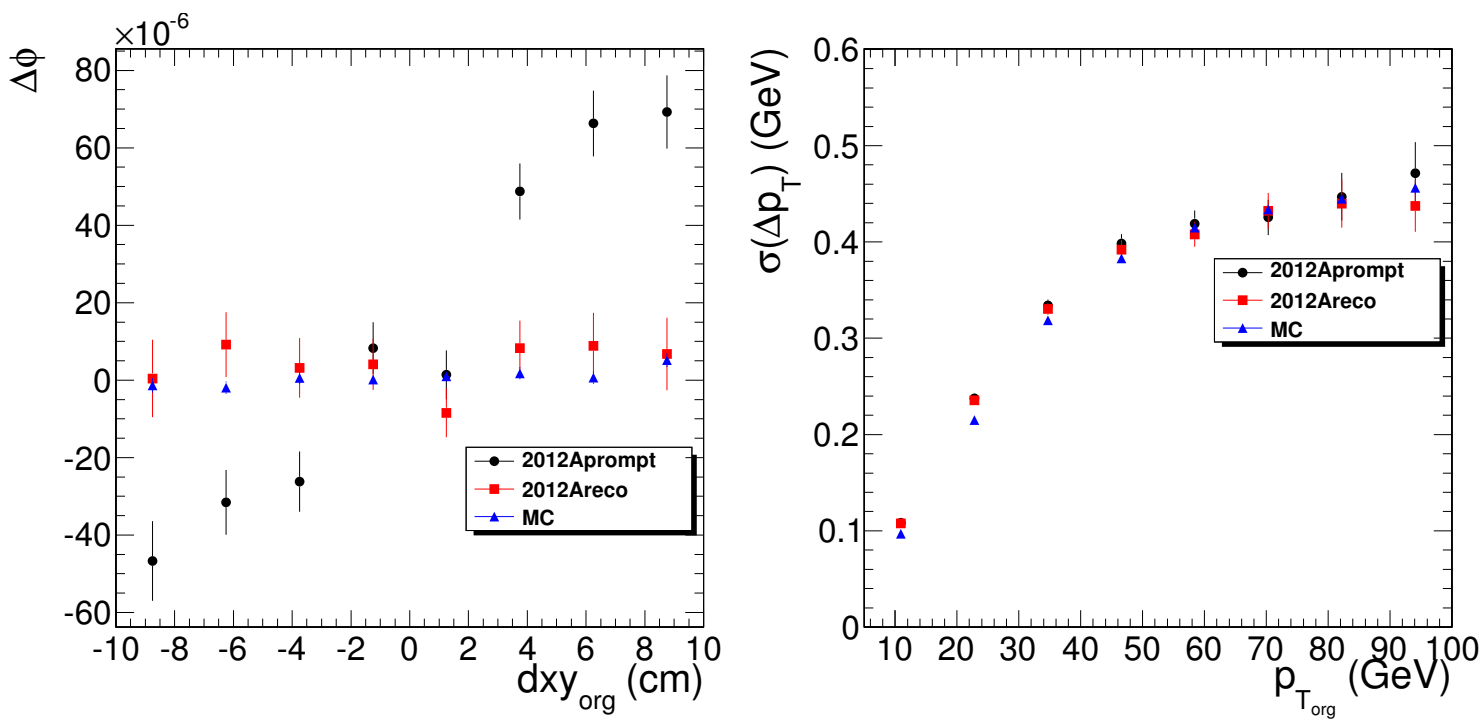

Figure 2.4: Profile plots of several reference geometries using cosmic tracks recorded during the 2012 Run A period. The left plot shows the difference in $d_{x y}$ between the two split tracks, $\Delta d_{x y}$ vs $\phi$. The right plot shows the width of the $\Delta p_{T}$ distribution, $\sigma\left(p_{T}\right)$, vs $p_{T}$.

relative resolution on $p_{T}$ varies from $.1 \mathrm{GeV}$ to $.45 \mathrm{GeV}$ for tracks with $p_{T}$ between 10 and $100 \mathrm{GeV}$. Cosmic tracks provide a unique source of very high $p_{T}$ muons. Using the track splitting procedure, these muons can be used to better understand tracking in this extreme phase space. In general, all track parameter errors can also be measured in bins of other variables, known as profile plots. The left plot of Figure 2.4 shows $d_{x y}$ in bins of $\phi$ for the 2012 Run A cosmic data. There is a significant improvement between the prompt and re-RECO geometries.

Profile plots are sensitive to structures like the ones shown in Figure 2.4 and can be used to gauge the presence of systematic misalignments of the tracker. In some cases, these misalignments are $\chi^{2}$ invariant, also known as weak modes. Some examples 


\section{CHAPTER 2. EXPERIMENTAL SETUP}
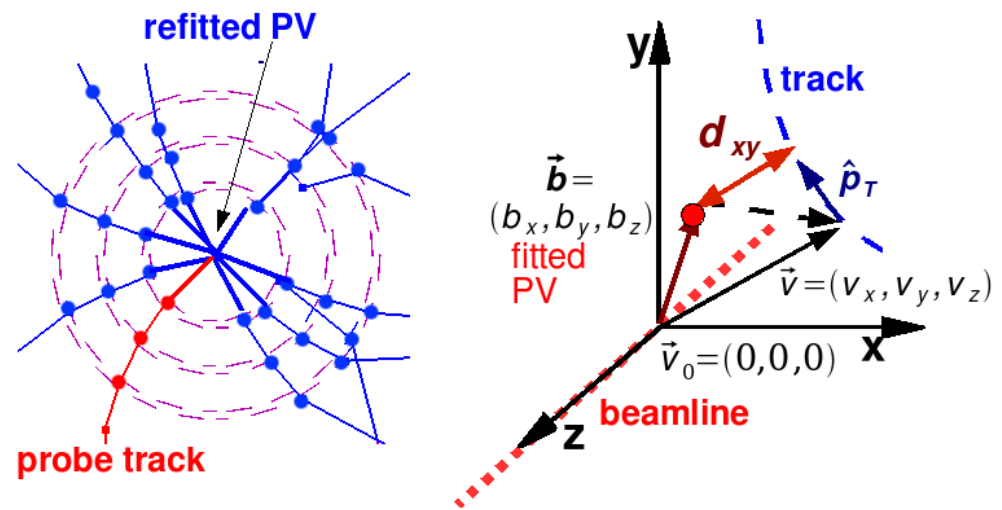

Figure 2.5: Diagram depicting the calculation of residuals used in the primary vertex validation.

include a systematic shift of modules in the $\mathrm{r}-\phi$ direction which is a function of $\phi$ itself. This type of deformation would result in the structure that is seen in the left plot of Figure 2.4 in the prompt geometry. In this case, the deformation is not a weak mode since the alignment procedure is sensitive to it and corrects the module positions accordingly. However, understanding similar deformations is important for assessing uncertainties in physics measurements.

The primary vertex validation uses the position of primary vertices as an estimator of the true impact parameters of an individual track. Residuals can be constructed from the difference between the primary vertex and a track's fitted impact parameter as demonstrated in Figure 2.5. If tracks truly originate from the vertex, then on average the above assumption will be true. However, individual tracks which pass through poorly aligned regions of the tracker will give larger residuals, thus providing a self consistent probe of the tracker geometry. 


\section{CHAPTER 2. EXPERIMENTAL SETUP}

Distributions of impact parameter residuals are sensitive to changes in the pixel modules. Figure 2.6 shows a number of residual distributions of the longitudinal impact parameter, $d_{z}$, in various bins of $\eta$ and $\phi$. Each bin represents tracks from a specific region of pixel module. The mean and RMS of these distributions, which are measured using double Gaussian fits, can provide useful information about systematic misalignments of the pixel barrel. In particular, this validation is sensitive to the presence of separation of the pixel half barrels, which tend to move when detector conditions change.

To quantify the separation of the pixel half barrels, the mean and width of the residual distributions are plotted as a function of $\phi$. If a separation between the two half barrels is present, it will cause a discontinuity at zero. Figure 2.7 shows an example plot of this using MC tracks with either the ideal geometry or a geometry in which the two half barrels have been purposefully shifted. The size of the discontinuity directly corresponds to the size of the physical separation.

The presence of a shift can have significant impact on vertex measurements, which can affect either efficiency of associating tracks with the primary vertex or efficiency of b-tagging. Thus, monitoring and correcting these deformations in time is critical. Figure 2.8 shows the measured separation of the pixel half barrels versus time before and after alignment parameters were determined. This procedure was critical for determining an effective alignment procedure by defining run ranges to perform independent alignments of large structures in order to correct the time dependence 

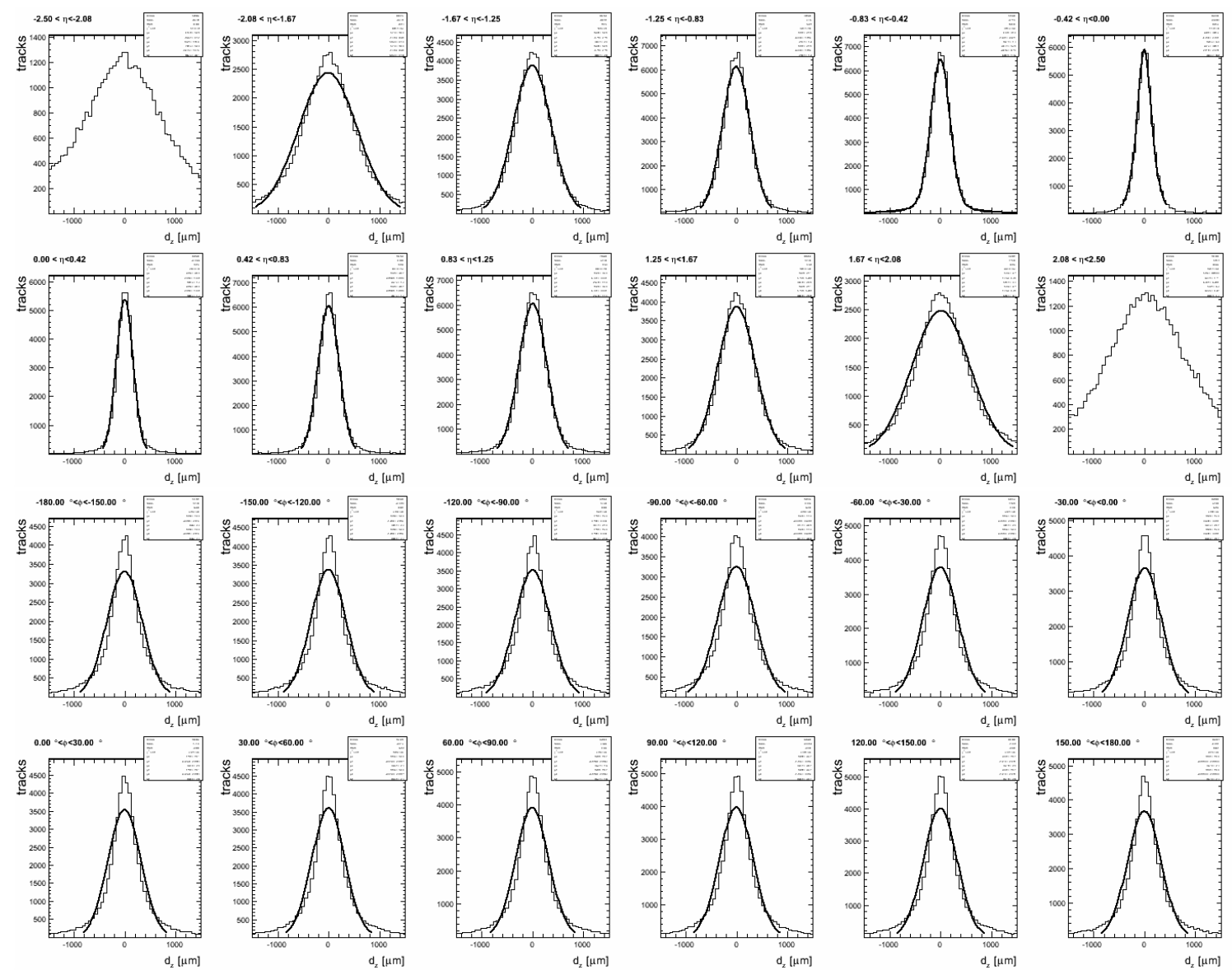

Figure 2.6: Residual transverse impact parameter distributions in bins of $\eta$ (top) and $\phi$ (bottom). 


\section{CHAPTER 2. EXPERIMENTAL SETUP}

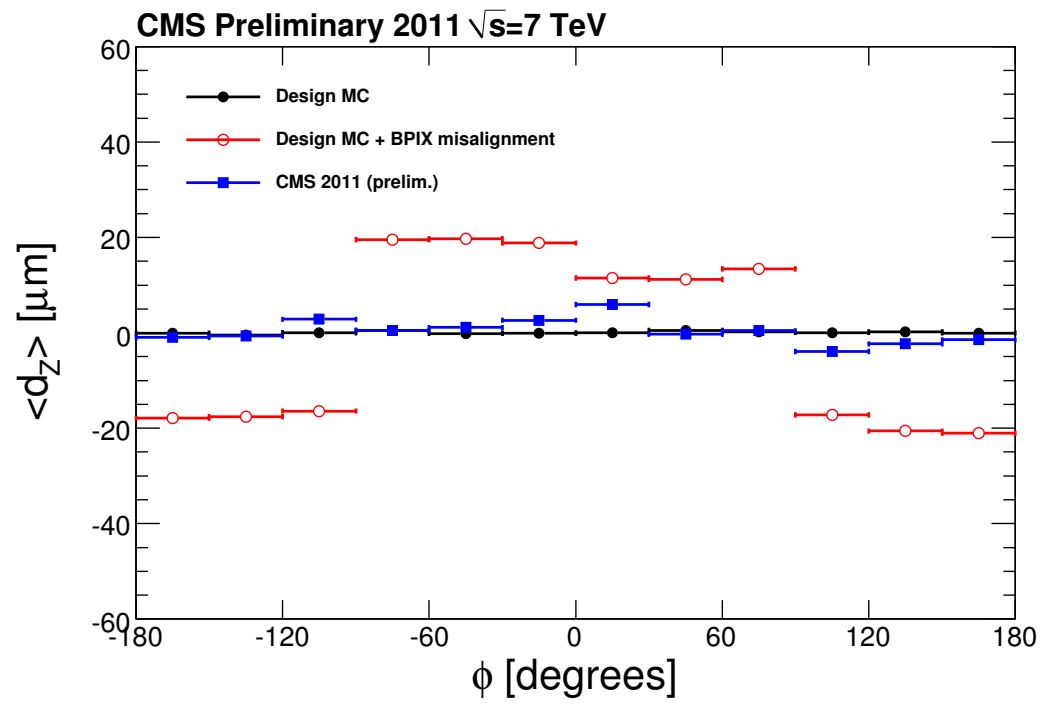

Figure 2.7: Distribution of mean and width of transverse impact parameter residuals in bins of the probe tracks azimuthal angle, $\phi$, for an ideal geometry (black), ideal geometry plus $40 \mu \mathrm{m}$ separation between the pixel half barrels (red), and the 2011 candidate geometry (blue).

seen. The red points in Figure 2.8 show that most of the time dependence is reduced to below 5-10 $\mu \mathrm{m}$.

\section{$2.3 \quad$ Summary}

The necessary but challenging environment provided by the LHC has produced higher collision energies than have ever previously been attained. This is critical for producing heavy resonances as well as increasing the phase space for producing intermediate mass resonances such as the Higgs boson. The design of CMS has allowed for high quality data collecting even in the midst of the high rates and high pileup 


\section{CHAPTER 2. EXPERIMENTAL SETUP}

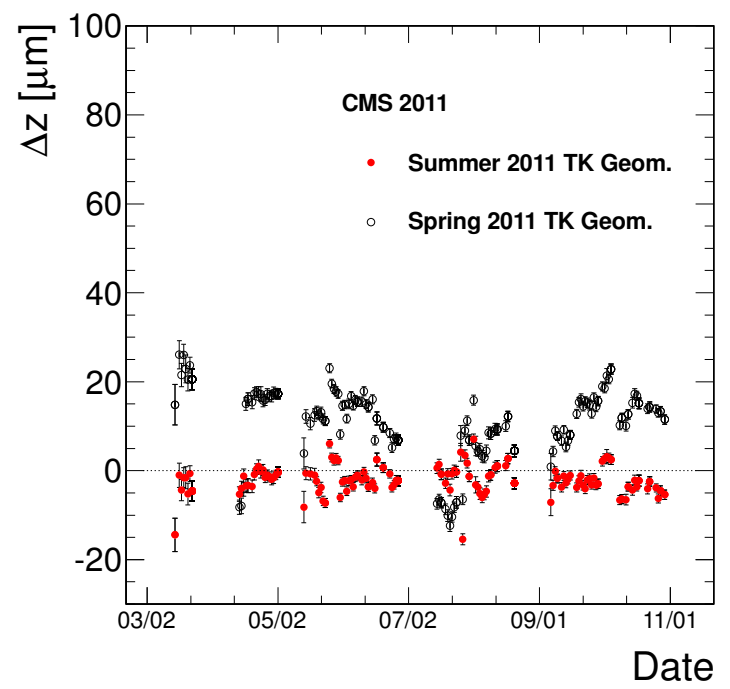

Figure 2.8: Measured separation between pixel half barrels versus time before and after alignment.

environments produced by the LHC. Offline validation, calibration, and alignment of the various sub-detectors is a critical aspect of the success of CMS.

The continued monitoring and adjustment of the tracker geometry using offline track-based alignment algorithms is critical for producing high precision track measurements. This will be critical to physics measurements, especially those related to Higgs boson searches. Since angular and mass distributions of the final state particles of resonances can be exploited for property measurements, to be discussed in Chapters 3 and 4, it is important to have tools like those mentioned above to monitor tracker performance using either collision tracks or cosmic tracks. 


\section{Chapter 3}

\section{Higgs Phenomenology at the LHC}

In the simplest incarnation of the Higgs mechanism, the Higgs boson mass is the only free parameter. Given the mass of the Higgs boson, the production cross section, branching fractions, and decay width can be calculated. Generally, the Higgs boson couples most strongly to the most massive particles in the SM. However, the mechanism for which the weak gauge bosons acquire mass and the fermions acquire mass in the SM is different. Thus, the coupling of the Higgs boson to fermions is proportional to the mass of the fermion while the coupling of the Higgs boson to the weak gauge bosons is proportional to the square of the gauge boson's mass. These features and the structure functions of the proton combine to produce the predictions shown in Figure 3.1 [33] for the production cross-section and branching fraction of the Higgs.

In this chapter, the terminology of the different production and decay channels are 

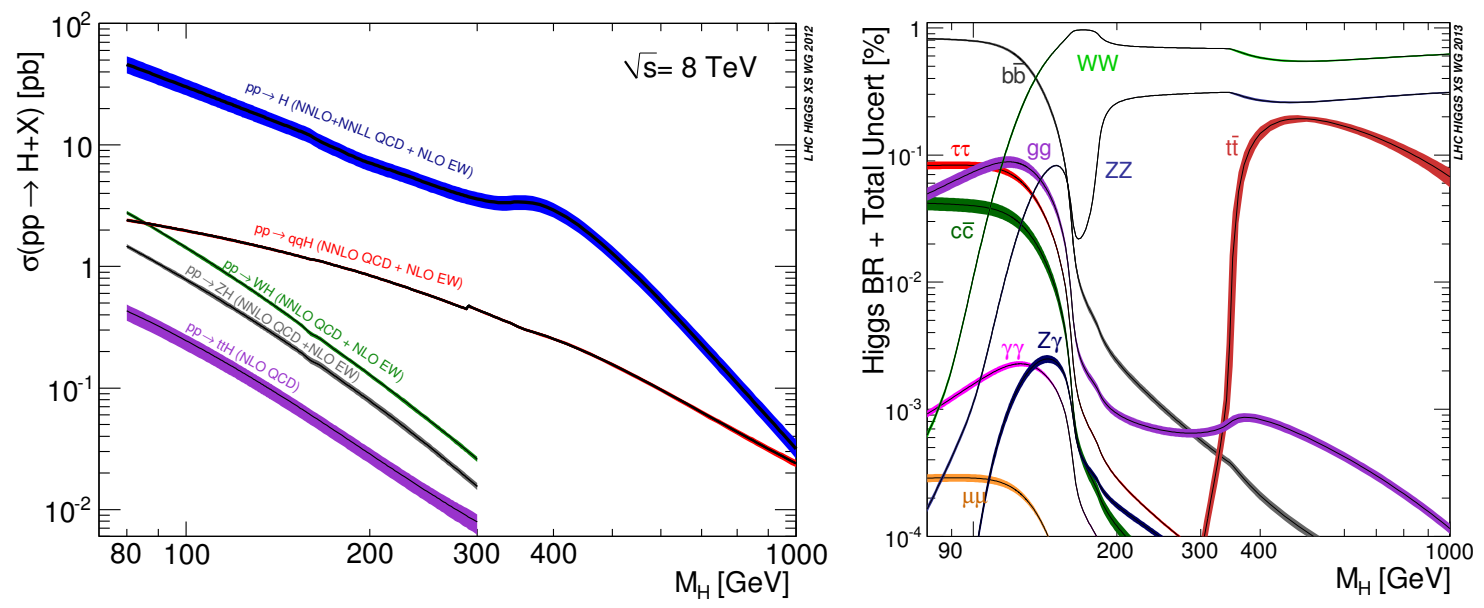

Figure 3.1: left: Higgs production cross section vs $m_{H}$ for different processes at $\sqrt{s}=8 \mathrm{TeV}$. right: Higgs branching ratios vs $m_{H}$. Both calculations are taken from the LHC Higgs cross section working group.

introduced as well as the experimental signatures for each. Kinematics of spin-0, spin1, and spin-2 resonances decaying to two vector bosons are introduced. Techniques for using decay kinematics for increasing signal sensitivity and performing property measurements are presented.

\subsection{Higgs Signatures}

\subsubsection{Gluon-gluon Fusion}

The gluon-gluon fusion production mechanism is responsible for $\sim 87 \%$ of Higgs events produced at the LHC, assuming $m_{H}=125 \mathrm{GeV}$ and $\sqrt{s}=8 \mathrm{TeV}$. This is due to the gluon-gluon cross section dominating over other initial states for the 


\section{CHAPTER 3. HIGGS PHENOMENOLOGY AT THE LHC}

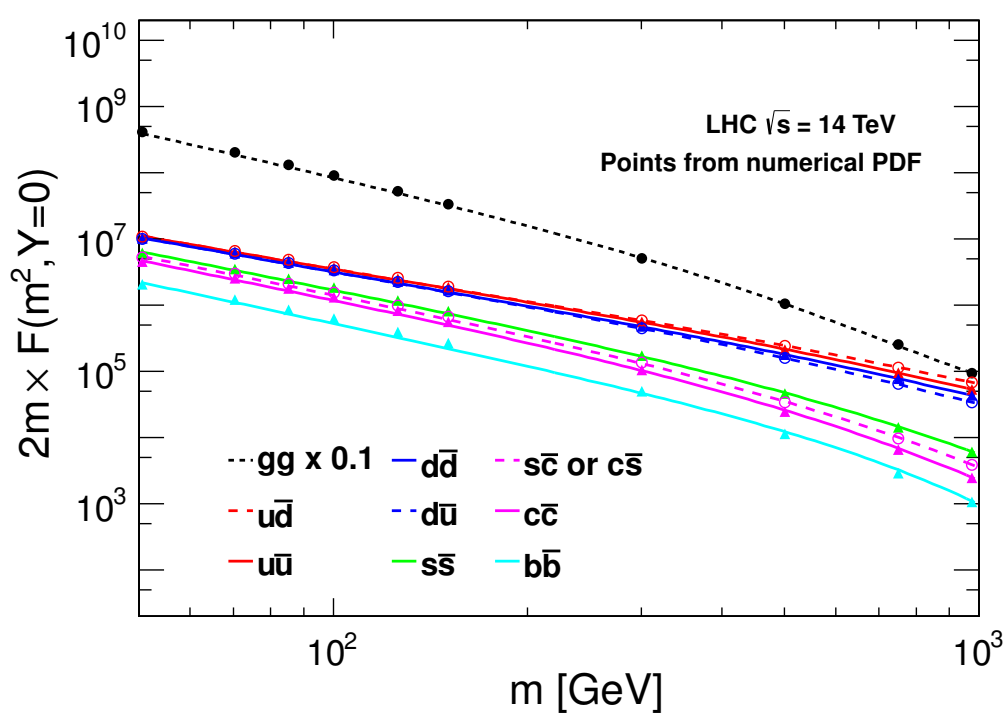

Figure 3.2: Distribution of parton factor, $\mathrm{F}(\mathrm{s}, \mathrm{Y}=0)$, showing the relative probability for producing resonances from gluon-gluon, or $q \bar{q}$ interaction for $\sqrt{s}=14 \mathrm{TeV}$.

relevant range of invariant masses, as shown in Figure 3.2 [34]. However, because the Higgs cannot couple to gluons directly, the interaction must be mediated through a loop, shown in Figure 3.3. The dominant contributions come from the heavy quarks, top and bottom quarks, which couple strongly to both gluons and the Higgs. The production cross section for this process varies from $3 \times 10^{-2} p b$ to $40 p b$ for Higgs masses between 80 and $1000 \mathrm{GeV}$ and $\sqrt{s}=8 \mathrm{TeV}$.

\subsubsection{Weak Vector Boson Fusion}

The Weak Vector Boson Fusion (VBF) production mechanism has the next to largest cross section at the LHC, depicted in Figure 3.4. The signature of this production mechanism is two energetic jets at high values of pseudorapidity. Because of 


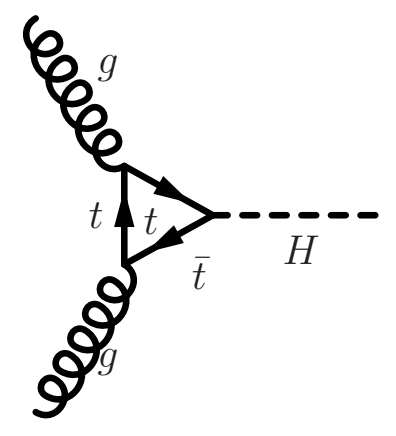

Figure 3.3: Feynman diagram depicting the leading contribution to gluon-gluon fusion production of a Higgs boson.

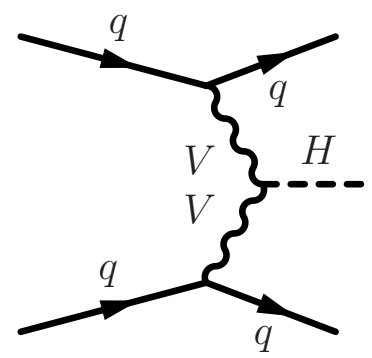

Figure 3.4: Feynman diagram depicting weak vector boson fusion production of a Higgs boson.

gluon radiation from next-to-leading order (NLO) and next-to-NLO (NNLO) QCD effects, gluon-gluon fusion events can also have this same signature. As such, event classes which attempt to distinguish the VBF production mechanism tend to have a large contamination from gluon-gluon fusion. Usually the kinematics of the spectator jets can be used to further isolate VBF-like events.

\subsubsection{Other Production Mechanisms}

Other production mechanisms produce Higgs bosons in association with either a weak gauge boson or top pair, both of which are depicted in Figure 3.5. In these cases 


\section{CHAPTER 3. HIGGS PHENOMENOLOGY AT THE LHC}
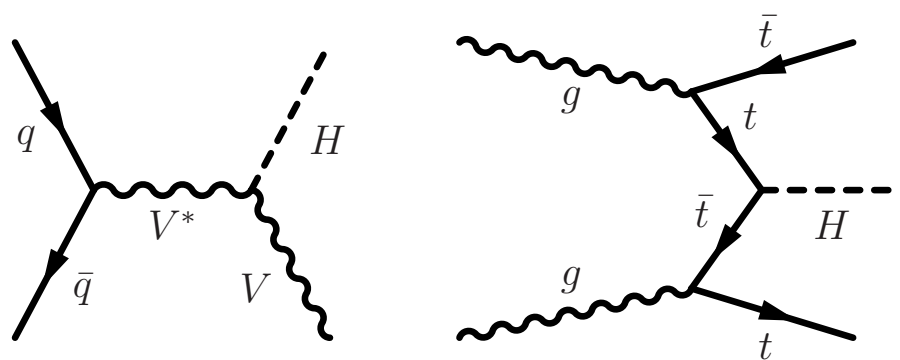

Figure 3.5: Feynman diagram depicting associated production (left) and $t \bar{t}$ fusion production of a Higgs boson.

either $\mathrm{W}$ or $\mathrm{Z}$ can be tagged or the presence of b-jets can be included. However, for $m_{H}=125 \mathrm{GeV}$, these processes only make up $5 \%$ of the total Higgs boson production cross section at the LHC. As such, having significant sensitivity to these production mechanisms requires very high amount of integrated luminosity, $\mathscr{O}\left(100 \mathrm{fb}^{-1}\right)$.

\subsubsection{Decay Channels}

The partial decay widths of the Higgs boson, just as with productions, are typically related to the mass of the decay products. As such, at low mass, where the production of weak gauge bosons is suppressed from phase-space effects, b-quarks are the dominant decay, making up $\sim 80 \%$ of the events. At high mass, the leading decays are to $\mathrm{W}$ and $\mathrm{Z}$ pairs. The SM has the particular feature that the $H \rightarrow \gamma \gamma$ and $H \rightarrow Z \gamma$ branching ratios are much smaller than the $H \rightarrow Z Z$ or $H \rightarrow W W$ branching ratios because the Higgs does not couple directly to massless particles. Thus, these processes are required to proceed through loops which would contain massive particles, usually either top quarks or $\mathrm{W}$ bosons. This is one of the most 


\section{CHAPTER 3. HIGGS PHENOMENOLOGY AT THE LHC}

distinguishing features which results in a large suppression of the $\gamma \gamma$ and $Z \gamma$ channels with respect to the $\mathrm{ZZ}$ and WW channels. The branching ratios versus $m_{H}$ are shown in Figure 3.1 for different decay channels.

Because of the distinct signature of ZZ, WW, and $\gamma \gamma$ decays, these channels are the most sensitive for discovering a Higgs-like resonance. The $4 \ell$ final state of the ZZ channel is especially promising because it is a high resolution, fully reconstructable channel with very small SM backgrounds.

\subsection{Kinematics of Scalar Resonances}

The simplest incarnation of the Higgs mechanism predicts one scalar boson with the simplest coupling to the SM fields. However, there are models which go beyond the minimal Higgs mechanism and predict other scalars which would couple differently to the SM fields. The most generic amplitude for a scalar which couples to two bosons is

$$
\begin{array}{r}
\mathscr{A}(X \rightarrow V V)=v^{-1}\left(g_{1} m_{v}^{2} \epsilon_{1}^{*} \epsilon_{2}^{*}+g_{2} f_{\mu \nu}^{*(1)} f^{*(2), \mu \nu}+\right. \\
\left.g_{3} f^{*(1), \mu \nu} f_{\mu \alpha}^{*(2)} \frac{q_{\nu} q^{\alpha}}{\Lambda^{2}}+g_{4} f_{\mu \nu}^{*(1)} f^{*(\tilde{2}), \mu \nu}\right),
\end{array}
$$

where $f$ and $\tilde{f}$ are the field strength tensor and the conjugate field strength tensor, $g_{i}$ are dimensionless couplings, $\epsilon_{i}$ are the polarization vectors of the vector bosons, $\Lambda$ denotes the scale where new physics could appear, $m_{v}$ is the mass of the vector 


\section{CHAPTER 3. HIGGS PHENOMENOLOGY AT THE LHC}

boson, and $\mathrm{q}$ is the momentum of the VV-system. This amplitude corresponds to three independent Lorentz structures and can be rewritten as,

$$
\mathscr{A}(X \rightarrow V V)=v^{-1} \epsilon_{1}^{* \mu} \epsilon_{2}^{* \nu}\left(a_{1} g_{\mu \nu} m_{X}^{2}+a_{2} q_{\mu} q_{\nu}+a_{3} \epsilon_{\mu \nu \alpha \beta} q_{1}^{\alpha} q_{2}^{\beta}\right)
$$

The translation between the couplings used in Equation 3.1 and those used in Equation 3.2 can be found in Equation 12 of Reference [1]. The SM Higgs boson couples to the weak vector boson only through the $a_{1}$ term and couples to photons through an effective coupling which is a combination of the $a_{1}$ and $a_{2}$ terms. A CP-odd scalar,

commonly referred to as a pseudoscalar, couples to the gauge bosons through the $a_{3}$ term.

The amplitude can be broken into several more specific amplitudes, known as helicity amplitudes, corresponding to the helicity states of the vector bosons, where the quantization axis is taken to be the direction of the VV decay in the resonance's rest frame. For a scalar resonance, there are only three non-zero helicity amplitudes out of the nine permutations,

$$
\begin{gathered}
A_{00}=-\frac{m_{X}^{2}}{v}\left(a_{1} \sqrt{1+x}+a_{2} \frac{m_{1} m_{2}}{m_{X}^{2}} x\right), \\
A_{++}=\frac{m_{X}^{2}}{v}\left(a_{1}+i a_{3} \frac{m_{1} m_{2}}{m_{X}^{2}} \sqrt{x}\right),
\end{gathered}
$$


CHAPTER 3. HIGGS PHENOMENOLOGY AT THE LHC

$$
A_{--}=\frac{m_{X}^{2}}{v}\left(a_{1}-i a_{3} \frac{m_{1} m_{2}}{m_{X}^{2}} \sqrt{x}\right)
$$

where $x$ is defined as

$$
x=\left(\frac{m_{X}^{2}-m_{1}^{2}-m_{2}^{2}}{2 m_{1} m_{2}}\right)^{2}-1 .
$$

While the above formulas apply to all bosonic decays of scalar resonances, $Z Z \rightarrow$ $4 \ell$ decays are particularly well suited for performing property measurements. This final state has very good momentum and angular resolution, low SM backgrounds, and sufficient complexity for all features of the most generic amplitude to be manifested.

A convenient basis of variables which can be used to fully describe $Z Z \rightarrow 4 \ell$ decays in the ZZ rest frame consists of the three invariant masses $\left(m_{X}, m_{1}\right.$, and $\left.m_{2}\right)$ and 5 angles, depicted in Figure 3.6. Each helicity amplitude has a distinct angular distribution while the magnitude of each helicity amplitude depends on the invariant masses of the two $\mathrm{Z}$ bosons and the resonance. Together these combine into the differential cross section according to

$$
\begin{array}{r}
\mathscr{P}\left(m_{1}, m_{2}, \vec{\Omega}\right) \propto\left|P_{V}\left(m_{1}, m_{2}\right)\right| \\
\times \frac{m_{1}^{3}}{\left(m_{1}^{2}-m_{v}^{2}\right)^{2}+m_{v}^{2} \Gamma_{v}^{2}} \times \frac{m_{2}^{3}}{\left(m_{2}^{2}-m_{v}^{2}\right)+m_{v}^{2} \Gamma_{v}^{2}} \\
\times \frac{d \Gamma_{J}\left(m_{1}, m_{2}, \vec{\Omega}\right)}{d \vec{\Omega}},
\end{array}
$$

where $\mathrm{q}$ is the magnitude of the vector boson momentum in the resonance's rest-frame. 


\section{CHAPTER 3. HIGGS PHENOMENOLOGY AT THE LHC}

For a spin-0 resonance, the angular distributions are given by

$$
\begin{array}{r}
\frac{d \Gamma_{J=0}}{\Gamma d \vec{\Omega}}=4\left|A_{00}\right|^{2} \sin ^{2} \theta_{1} \sin ^{2} \theta_{2} \\
+\left|A_{++}\right|^{2}\left(1-2 A_{f 1} \cos \theta_{1}+\cos ^{2} \theta_{1}\right)\left(1+2 A_{f 2} \cos \theta_{2}+\cos ^{2} \theta_{2}\right) \\
+\left|A_{--}\right|^{2}\left(1+2 A_{f 1} \cos \theta_{1}+\cos ^{2} \theta_{1}\right)\left(1-2 A_{f 2} \cos \theta_{2}+\cos ^{2} \theta_{2}\right) \\
+4\left|A_{00}\right|\left|A_{++}\right|\left(A_{f 1}+\cos \theta_{1}\right) \sin \theta_{1}\left(A_{f 2}+\cos \theta_{2}\right) \sin \theta_{2} \cos \left(\Phi+\phi_{++}\right) \\
+4\left|A_{00}\right|\left|A_{--}\right|\left(A_{f 1}-\cos \theta_{1}\right) \sin \theta_{1}\left(A_{f 2}-\cos \theta_{2}\right) \sin \theta_{2} \cos \left(\Phi-\phi_{--}\right) \\
+2\left|A_{++}\right|\left|A_{--}\right| \sin ^{2} \theta_{1} \sin ^{2} \theta_{2} \cos \left(2 \Phi-\phi_{--}+\phi_{++}\right)
\end{array}
$$

where $A_{f i}$ are the $Z \rightarrow f \bar{f}$ amplitudes which can be found in Reference [1]. The resulting differential cross section is parameterized in terms of the underlying couplings. The angular and mass distributions for several types of scalar models are shown in Figures 3.7 and 3.8. The red and blue distributions correspond to a SM Higgs and pseudoscalar resonances. The green distributions correspond to a scalar model in which the resonance couples to the vector boson only through the $g_{2}$ term of Equation 3.1, referred to here as the $0_{h}^{+}$model. All resonance models are simulated with JHUGen. A description of this generator and the models used here are provided in $[1,35]$. Thus, these three models represent the three independent Lorentz structures of the most generic scalar-vector-vector amplitude.

In principle, a mixture of these terms can occur. In fact, there is a small but negligible contribution from the $g_{2}$ term in the SM from higher order electroweak 


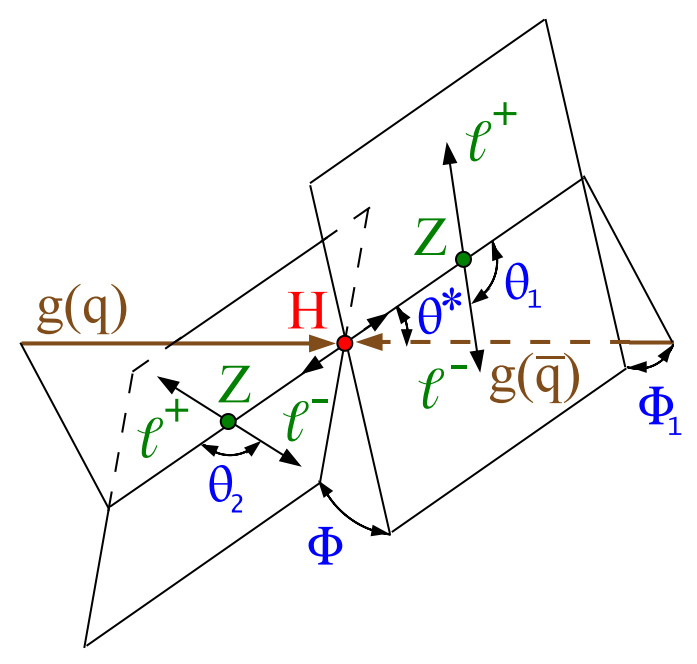

Figure 3.6: Diagram depicting $H \rightarrow Z Z \rightarrow 4 \ell$ decays and definition of angles which describe the kinematics of these decays.
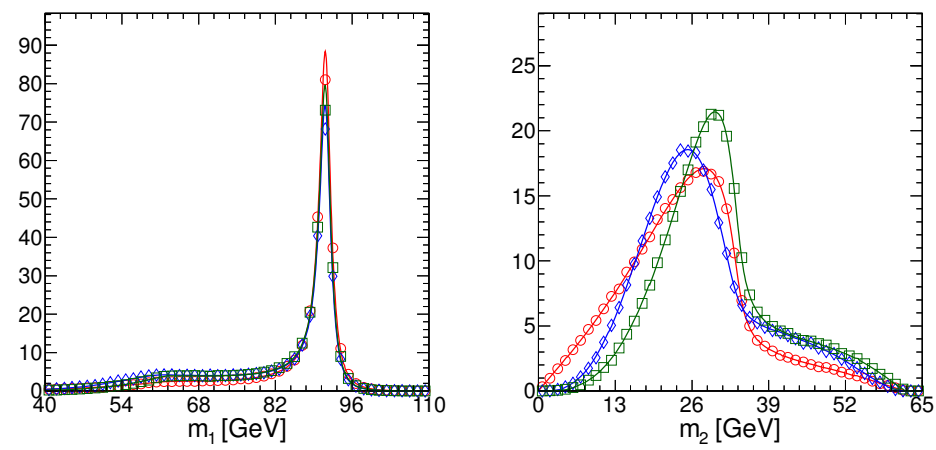

Figure 3.7: Distributions of the $\mathrm{Z}$ boson masses. The smaller of the two masses is plotted on the right, while the larger of the two masses is plotted on the left. Markers show simulated events; lines are projections of the analytical distribution described above. Red lines/circles correspond to a SM Higgs, blue lines/diamonds, a pseudoscalar, and green lines/square, a CP-even scalar produced from higher dimension operators. 
CHAPTER 3. HIGGS PHENOMENOLOGY AT THE LHC
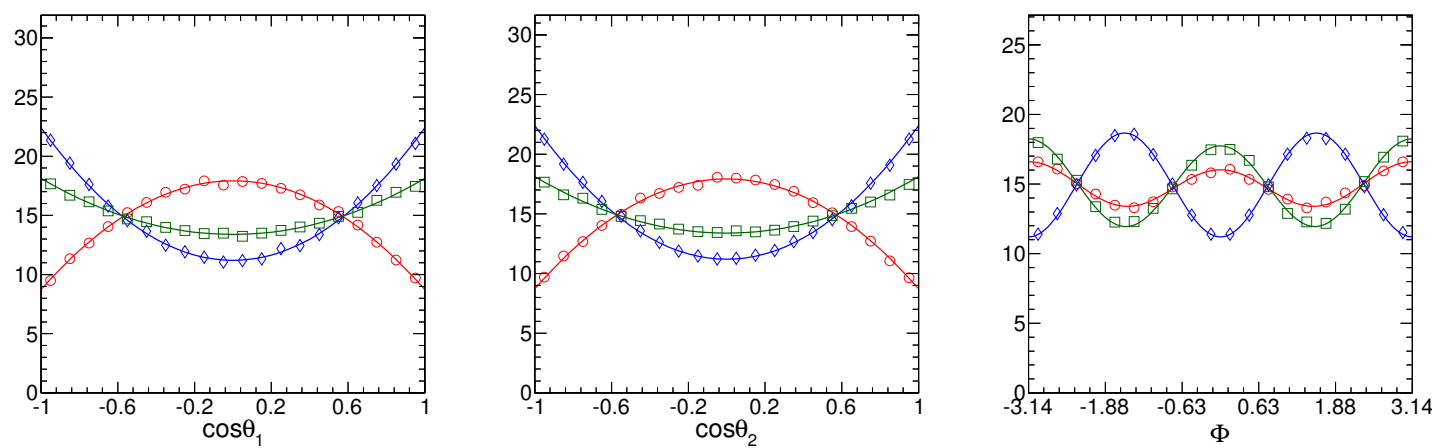

Figure 3.8: Distributions of helicity angles, $\cos \theta_{1}$ (left), $\cos \theta_{2}$ (middle), and $\Phi$ (right). Markers show simulated events; lines are projections of the analytical distribution described above. Red lines/circles correspond to a SM Higgs, blue lines/diamonds, a pseudoscalar, and green lines/square, a CP-even scalar produced from higher dimension operators.

corrections. In various extensions to the SM, e.g. 2 Higgs doublet models, multiple scalars exist with different $\mathrm{CP}$ properties. It is even possible that CP-violating interactions could exist. Constraining the contribution from either the $g_{2}$ or $g_{4}$ term of the amplitude can be more aptly formulated through a reparametrization of the HZZ amplitude. Starting from the three complex couplings, $g_{1}, g_{2}$, and $g_{4}$, four real parameters can be defined

$$
\begin{gathered}
f_{i}=\frac{\left|g_{i}\right|^{2} \sigma_{i}}{\left|g_{1}\right|^{2} \sigma_{1}+\left|g_{2}\right|^{2} \sigma_{2}+\left|g_{4}\right| \sigma_{4}} \\
\phi_{g i}=\arg \left(\frac{g_{i}}{g_{1}}\right),
\end{gathered}
$$




\section{CHAPTER 3. HIGGS PHENOMENOLOGY AT THE LHC}

for $i=2,4$. In the above formula, $\sigma_{i}$ is the cross section of the process corresponding to $g_{i}=1$ and $g_{\neq i}=0$. The $f_{g i}$ parameters represent an effective fraction of events resulting from the corresponding term of the amplitude. In the case where there is no interference, this interpretation is exact. This parametrization factorizes out the total cross section, assuming that it will be measured separately. These variables are also straight forward measurables for experiments where rates are directly measured, as will be discussed in later sections. In Chapters 4, a slightly different notation will be used for the fractions and the translation, $f_{a 3}=f_{g 4}$ and $f_{a 2}=f_{g 2}$ should be applied.

Similar differential cross sections can be calculated for a generic spin-1 or spin2 resonance decaying to two $\mathrm{Z}$ bosons [1]. Figures 3.9, 3.10, and 3.11 show two choice vector resonance models. Figures 3.12, 3.13, and 3.14 show three choice tensor resonance models. The couplings used to define each of these models are shown in Table 3.1.

\subsubsection{Variables for Property Measurements}

Several extensions to the SM discussed previously in Chapter 1, can result in ZZ resonances. Consequently, understanding the spin and $\mathrm{CP}$ of any new resonance discovered at the LHC will be critical to understanding its role in nature. An efficient way of constraining resonance properties is to use compact variables to isolate specific properties. Such a variable can be built from either the square of the matrix element for two processes, or equivalently, the differential cross section defined above, 


\section{CHAPTER 3. HIGGS PHENOMENOLOGY AT THE LHC}
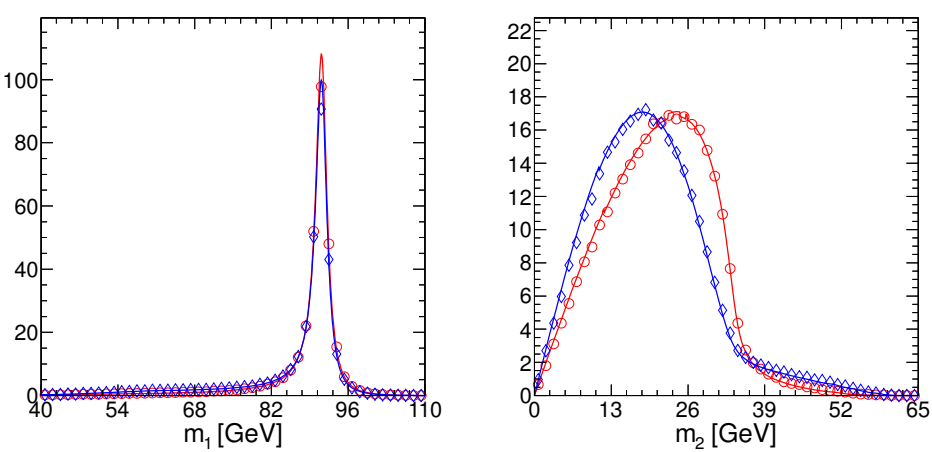

Figure 3.9: Distributions of the $\mathrm{Z}$ boson masses. The smaller of the two masses is plotted on the right, while the larger of the two masses is plotted on the left. Markers show simulated events; lines are projections of the analytical distribution described above. Red lines/circles correspond to a CP-even vector, blue lines/diamonds to a $\mathrm{CP}$-odd vector.
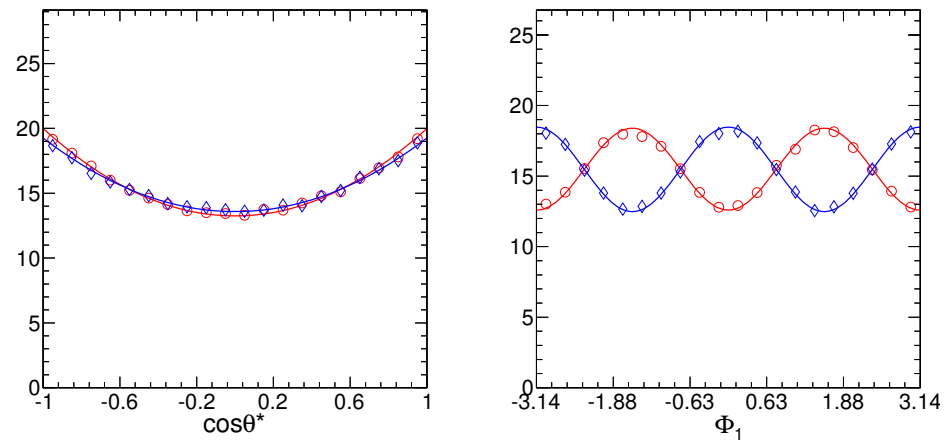

Figure 3.10: Distributions of the production angles, $\cos \theta^{*}$ (left) and $\Phi_{1}$ (right). Markers show simulated events; lines are projections of the analytical distribution described above. Red lines/circles correspond to CP-even vector, blue lines/diamonds to a CPodd vector. 


\section{CHAPTER 3. HIGGS PHENOMENOLOGY AT THE LHC}
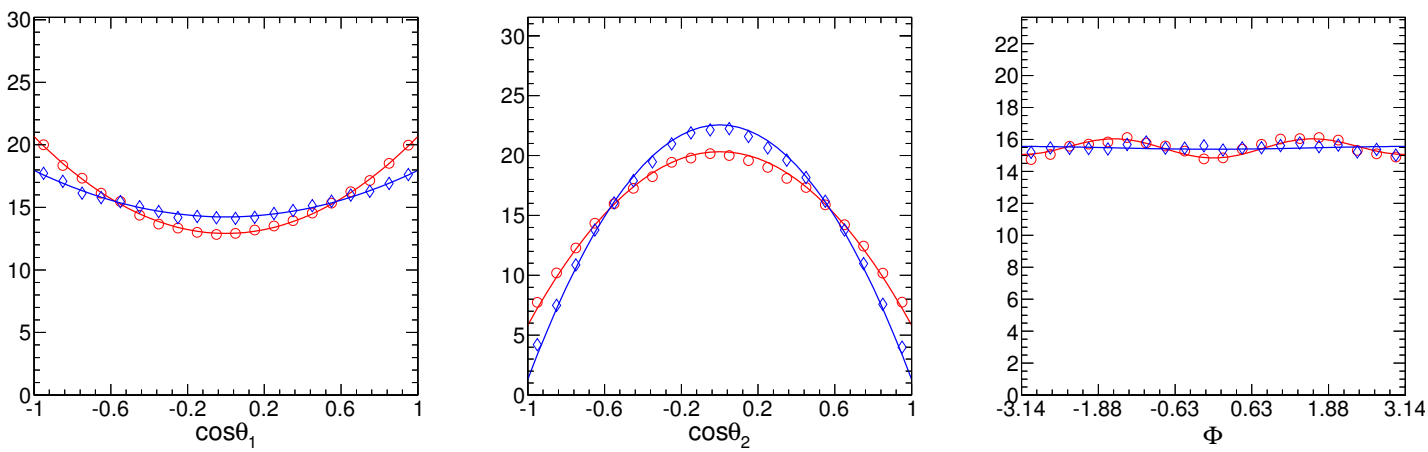

Figure 3.11: Distributions of the helicity angles, $\cos \theta_{1}$ (left), $\cos \theta_{2}$ (middle), and $\Phi$ (right). Markers show simulated events; lines are projections of the analytical distribution described above. Red lines/circles correspond to CP-even vector, blue lines/diamonds to a $\mathrm{CP}$-odd vector.
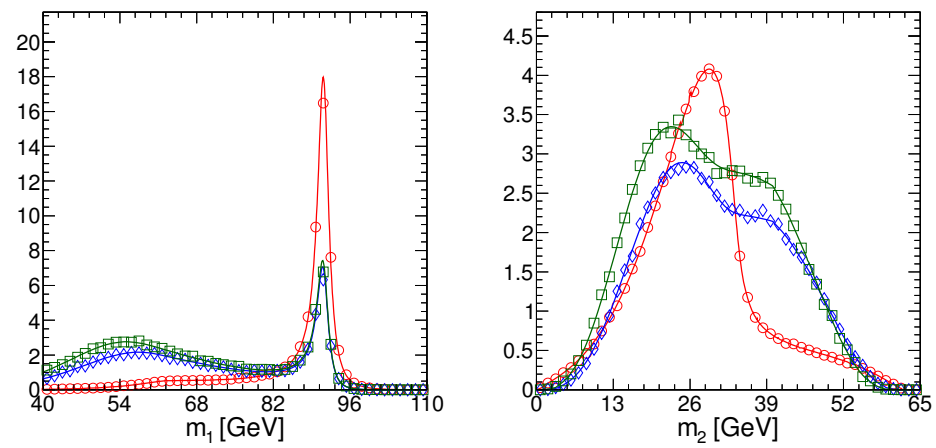

Figure 3.12: Distributions of the $\mathrm{Z}$ boson masses. The smaller of the two masses is plotted on the right, while the larger of the two masses is plotted on the left. Markers show simulated events; lines are projections of the analytical distribution described above. Red lines/circles correspond to a minimal coupling graviton, blue lines/diamonds to a CP-odd tensor, and green lines/square to a CP-even tensor produced from higher dimension operators. 


\section{CHAPTER 3. HIGGS PHENOMENOLOGY AT THE LHC}
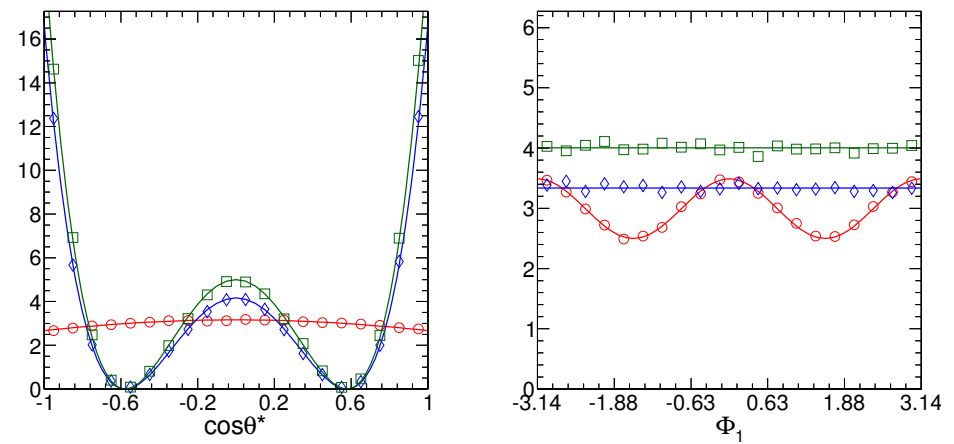

Figure 3.13: Distributions of the production angles, $\cos \theta^{*}$ (left) and $\Phi_{1}$ (right). Markers show simulated events; lines are projections of the analytical distribution described above. Red lines/circles correspond to a minimal coupling graviton, blue lines/diamonds to a CP-odd tensor, and green lines/square to a CP-even tensor produced from higher dimension operators.
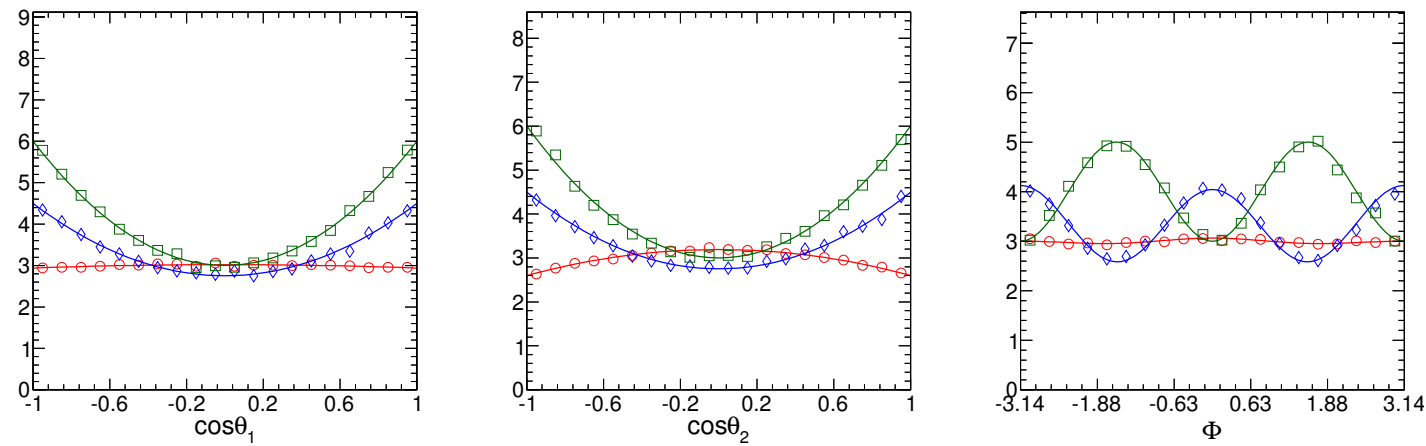

Figure 3.14: Distributions of the helicity angles, $\cos \theta_{1}$ (left), $\cos \theta_{2}$ (middle), and $\Phi$ (right). Markers show simulated events; lines are projections of the analytical distribution described above. Red lines/circles correspond to a minimal coupling graviton, blue lines/diamonds to a CP-odd tensor, and green lines/square to a CPeven tensor produced from higher dimension operators. 


\section{CHAPTER 3. HIGGS PHENOMENOLOGY AT THE LHC}

\begin{tabular}{cccc}
\hline \hline scenario & X prod & $X \rightarrow V V$ decay & comments \\
\hline $0_{m}^{+}$ & $g g \rightarrow X$ & $g_{1} \neq 0$ & SM Higgs boson \\
$0_{h}^{+}$ & $g g \rightarrow X$ & $g_{2} \neq 0$ & scalar with higher-dim operators \\
$0^{-}$ & $g g \rightarrow X$ & $g_{4} \neq 0$ & pseudoscalar \\
$1^{+}$ & $q \bar{q} \rightarrow X$ & $b_{2} \neq 0$ & exotic pseudovector \\
$1^{-}$ & $q \bar{q} \rightarrow X$ & $b_{1} \neq 0$ & exotic vector \\
$2_{m}^{+}$ & $g_{1}^{(2)}=g_{5}^{(2)} \neq 0$ & $g_{1}^{(2)}=g_{5}^{(2)} \neq 0$ & tensor with min couplings \\
$2_{b}^{+}$ & $g_{1}^{(2)}=\neq 0$ & $g_{5}^{(2)} \neq 0$ & bulk tensor with min couplings \\
$2_{h}^{+}$ & $g_{4}^{(2)} \neq 0$ & $g_{4}^{(2)} \neq 0$ & tensor with higher-dim operators \\
$2_{h}^{-}$ & $g_{8}^{(2)} \neq 0$ & $g_{8}^{(2)} \neq 0$ & "pseudotensor" \\
\hline \hline
\end{tabular}

Table 3.1: List of alternative signal models to be tested against the SM Higgs hypothesis along with a description of the their couplings to ZZ. Amplitude parametrization for spin-0 resonances is given in Equation 3.1; parametrizations for spin-1 and spin-2 resonances are given in Equations 16 and 18 elsewhere [1].

according to

$$
\mathscr{D}_{J^{P}}=\left(1+\frac{\mathscr{P}_{J^{P}}\left(m_{1}, m_{2}, \vec{\Omega} \mid m_{4 \ell}\right)}{\mathscr{P}_{0^{+}}\left(m_{1}, m_{2}, \vec{\Omega} \mid m_{4 \ell}\right)}\right)^{-1}
$$

where $\mathscr{P}_{J^{P}}$ and $\mathscr{P} 0^{+}$are evaluated using the corresponding matrix elements. These types of variables use ideal distributions to isolate the relevant kinematic differences between two choice models. For $Z Z \rightarrow 4 \ell$ events these variables will be close to optimal since acceptance effects will cancel when calculating ratios and resolution effects are relatively small (see Section 2.2.6.3). In other channels, steps can be taken to mitigate the effects of resolution (see Section 4.1).

An accurate description of the detector level distribution of $\mathscr{D}_{J^{P}}$ must be modeled. Simulated Monte Carlo (MC) events can be used, including all detector simulations, reconstruction algorithms, and analysis selections, to model the shape of these discriminants. Thus, MC simulations can effectively be used to model the appropriate 


\section{CHAPTER 3. HIGGS PHENOMENOLOGY AT THE LHC}

transfer function for a given analysis. The discriminant $\mathscr{D}_{J^{P}}$ can be used either as an additional selection variable, or for constructing likelihoods. This process of building discriminants from kinematic distributions using a matrix element calculation paired with MC simulations is known as the Matrix Element Likelihood Approach (MELA).

Even with a relatively small number of signal events, the MELA technique can be used to perform hypothesis separation to rule out definite non-SM signals. For example, the variable $\mathscr{D}_{0-}$ can be used to isolate the relevant properties that distinguish a SM Higgs from a purely CP-odd scalar. The SM Higgs and pseudoscalar distribution of $\mathscr{D}_{0-}$ for ideal MC is shown in Figure 3.15. The separation between these two models can be quantified using Neyman-Pearson hypothesis testing. In this way, the compatibility of data with respect to either the null hypothesis (always the SM Higgs hypothesis) or the alternative hypothesis can be quantified. Other models, such as spin-1 or spin-2 models, can be tested using variables analogous to $\mathscr{D}_{0-}$. A list of models which will be used in Section 4.2 to perform such tests are listed in Table 3.1 along with a description.

Certain discriminants have properties which allow them to be efficiently used to measure model parameters. Assuming $f_{g 2}=0, f_{g 4}$ can be measured directly using $\mathscr{D}_{0-}$. Figure 3.15 shows this discriminant for both the SM Higgs (solid black line), a pseudoscalar (dashed black line), and a mixed parity model corresponding to $f_{g 4}=0.5$ (red line). All of the mixed parity samples can be described by a weighted sum of 


\section{CHAPTER 3. HIGGS PHENOMENOLOGY AT THE LHC}
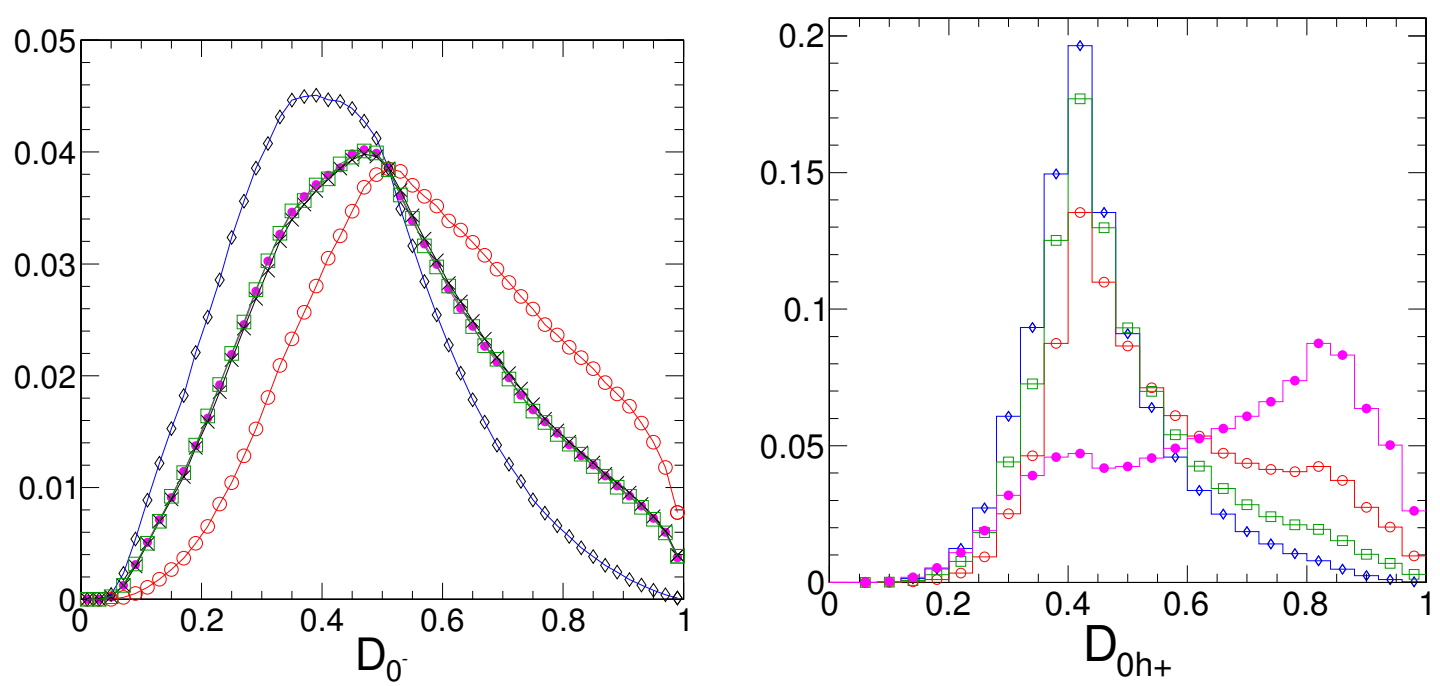

Figure 3.15: Distributions of $D_{0-}$ (left) and $D_{0_{h}^{+}}$(right) for various scalar models. A SM Higgs (open red circles), a pseudoscalar (blue diamonds), and two mixed states corresponding to $f_{g i}=0.5$ with $\phi_{g i}=0$ (green squares) and $\phi_{g i}=\pi / 2$ (closed magenta circles) are shown. For the left plot, $i=4$. For the right plot, $i=2$. Black crosses show the distribution of the mixed states with no interference. 


\section{CHAPTER 3. HIGGS PHENOMENOLOGY AT THE LHC}

the SM Higgs distribution and the pseudoscalar distribution (blue line),

$$
\begin{array}{r}
\mathscr{P}\left(D_{0-} \mid f_{g 4}\right)=\left|\mathscr{A}_{0^{+}}\right|^{2}+\left|\mathscr{A}_{0_{-}}\right|^{2}+2 \operatorname{Re}\left(\mathscr{A}_{0^{+}}^{*} \mathscr{A}_{0_{-}}\right) \\
\simeq\left(1-f_{g 4}\right) \mathscr{P}_{0^{+}}\left(\mathscr{D}_{0-}\right)+f_{g 4} \mathscr{P}_{0-}\left(\mathscr{D}_{0-}\right) .
\end{array}
$$

$\mathscr{P}_{0^{+}}$and $\mathscr{P}_{0-}$ represent the differential cross section of the SM Higgs model and the pseudoscalar, respectively. Thus, Equation 3.9 explicitly neglects interference, but Figure 3.15 demonstrates that $\mathscr{D}_{0-}$ is insensitive to the interference and the relative phase between $\mathscr{A}_{0^{+}}$and $\mathscr{A}_{0-}$.

In contrast, the $\mathscr{D}_{0 h+}$ discriminant cannot be used measure $f_{g 2}$. Figure 3.15 shows that the interference between the $g_{1}$ and $g_{2}$ terms cannot be neglected and depends strongly on the $\phi_{g 2}$. This implies that more advanced techniques which can fit for both the fraction and the phase simultaneously will be needed to constrain this parameter.

Similar variables can be constructed to help discriminate signal effects from SM background events,

$$
\mathscr{D}_{b k g}^{k i n}=\left(1+\frac{\mathscr{P}_{b k g}\left(m_{1}, m_{2}, \vec{\Omega} \mid m_{4 \ell}\right)}{\mathscr{P}_{s i g}\left(m_{1}, m_{2}, \vec{\Omega} \mid m_{4 \ell}\right)}\right)^{-1}
$$

Analytical calculations for the continuum ZZ process are taken from Reference [36,37]. Typically, invariant mass distributions are used in resonances searches. As will be shown in Chapter 4 , variables similar to $D_{b k g}$ have proven to provide a significant increase in sensitivity to Higgs-like events if used in conjunction with the relevant 


\section{CHAPTER 3. HIGGS PHENOMENOLOGY AT THE LHC}

invariant mass distributions. It should be noted that these variables are important for properties as well; understanding properties of signal events first requires good sensitivity to signal events.

\subsection{Summary}

Understanding the role in electroweak symmetry breaking of any Higgs-like resonance can be divided into two classes of measurements: measuring relative cross sections in various production and decay channels, and measuring kinematic distributions within a given channel. These sets of measurements provide complementary information. Kinematic distributions can be used to build kinematic distributions to either perform hypothesis testing to constrain properties or to measure certain model parameters. Kinematic distributions will eventually allow for measurements of the effective couplings between a resonance and the $\mathrm{Z}$ bosons. In addition, the tools presented above can be used to maximize sensitivity to signal-like events. Two implementations of these ideas will be presented in the following chapter. However, these tools are quite general and apply to other production and decay processes as well as other colliders, e.g. $e^{+} e^{-} \rightarrow Z * \rightarrow Z H$. Chapter 5 will address the prospects of applying these tools to other processes. 


\section{Chapter 4}

\section{Higgs Searches with ZZ decays}

The ZZ channel is particularly well suited for Higgs search, especially at high mass $\left(m_{H}>200 \mathrm{GeV}\right)$ where the branching ratios to $\mathrm{WW}$ and $\mathrm{ZZ}$ are dominant. The $\mathrm{ZZ}$ channel has the advantage that there are several fully reconstructable final states: the $4 \ell$ final state and the $2 \ell 2 q$ final state. While the $4 \ell$ channels has very good mass resolution and low background, it suffers from low branching ratios. In complement, the $2 \ell 2 q$ channel has considerably larger background and mass resolution, but the hadronic branching ratio for the $\mathrm{Z}$ is large, $\mathscr{B}(Z Z \rightarrow 2 \ell 2 q) / \mathscr{B}(Z Z \rightarrow 4 \ell) \sim 20$. The $4 \ell$ channel is expected to provide high sensitivity to a broad range of Higgs mass hypotheses, while the dominant sensitivity for $2 \ell 2 q$ will occur at high mass and only moderate sensitivity can be achieved below the ZZ kinematic threshold.

In this chapter, two analyses will be presented in which Higgs searches are performed over the entire range of Higgs masses. The first section will concentrate on the 


\section{CHAPTER 4. HIGGS SEARCHES WITH ZZ DECAYS}

semileptonic final state. Novel analysis techniques to reduce and control for the background are presented. The sensitivity is found to be competitive with that expected from the $4 \ell$ channel. The second section will discuss Higgs searches in the context of the $4 \ell$ final state in which a significant excess of events has been observed consistent with a narrow width neutral bosonic resonance. The corresponding cross section of the excess is compared to that of SM Higgs expectation and property measurements are performed using event kinematics to constrain both the spin and parity of the observed resonance. 
CHAPTER 4. HIGGS SEARCHES WITH ZZ DECAYS

\subsection{Semi-leptonic decay channel}

The semileptonic final state of the ZZ channel is studied in two different kinematic regions, the low mass region $\left(125<m_{2 \ell 2 q}<170 \mathrm{GeV}\right)$ and the high mass region $\left(183<m_{2 \ell 2 q}<800 \mathrm{GeV}\right)$. Because of the small ZZ branching ratio expected from the SM Higgs, the intermediate range $\left(170<m_{2 \ell 2 q}<183 \mathrm{GeV}\right)$ is not considered in this analysis.

\subsubsection{Event Simulation}

The analysis strategy, including selections and data-driven background estimations, were optimized and validated on MC simulations. Signal samples are generated with POWHEG [38-40] and JHUGen [35]. Inclusive Z production is generated with either MADGRAPH 4.4.12 [41] or ALPGEN 2.13 [42]. Continuum diboson production, ZZ, WW, and ZW, samples are generated with PYTHIA 6.4.22 [43]. Top backgrounds are generated with either MADGRAPH 4.4.12 or POWHEG. Parton distribution functions are modeled using CTEQ6 [44] at leading order and CT10 [45] at next-to-leading order (NLO). Parton showering and hadronization is modeled with PYTHIA while detector response is simulated with a CMS specific implementation of GEANT4 [46]. A full list of the MC samples used is shown in table 4.1 along with the cross section for each process. MC simulations are corrected for mismodeling of pileup and any relative efficiencies found between data and MC through tag and probe measurements 
CHAPTER 4. HIGGS SEARCHES WITH ZZ DECAYS

\begin{tabular}{l|c|c|c}
\hline \hline Name & Generator & $\Gamma[\mathrm{GeV}]$ & $\sigma \times \mathscr{B}_{Z Z} \times \mathscr{B}_{2 l 2 q}[\mathrm{fb}]$ \\
\hline SM Higgs & POWHEG & & \\
$m_{H}=130-600$ & JHUGen & $0.0081-123$ & $125.129-14.7312$ \\
\hline \hline Name & Generator & & $\sigma_{L O}\left(\sigma_{N L O}\right)[\mathrm{pb}]$ \\
\hline \hline Z+jets & MADGRAPH & - & $2289(3084)$ \\
$Z+$ jets & SHERPA & - & 2943 \\
$t \bar{t}$ & PYTHIA & - & $94(157.5)$ \\
$t \bar{t}$ & POWHEG & - & $15.86(16.7)$ \\
$Z Z \rightarrow$ anything & PYTHIA & - & $4.30(5.9)$ \\
$W W \rightarrow$ anything & PYTHIA & - & $10.4(18.3)$ \\
$Z W \rightarrow$ anything & PYTHIA & - & $27.8(42.9)$ \\
\hline \hline
\end{tabular}

Table 4.1: Table summarizing MC simulations used to model signal and each of the different SM background along with their cross sections.

\subsubsection{Event Reconstruction, Selection, and Cate-}

\section{gorization}

Reconstruction of electron, muons, and jets is done using standard CMS algorithms. More details can be found elsewhere [47] and references therein. Only events which contain two oppositely charge leptons, either electrons or muons, and two jets are considered in this analysis. Both leptons flavors are required to have transverse momentum, $p_{T}$, greater than $20 \mathrm{GeV}$ and $10 \mathrm{GeV}$ for the leading and subleading $p_{T}$, respectively. For events which are used in the high mass analysis this constraint is tightened to $p_{T}>40,20 \mathrm{GeV}$. Only muons (electrons) in the pseudorapidity range $|\eta|<2.4(2.5)$. Electrons from the gap between the barrel and endcap region are also excluded. These selections not only serve as a rudimentary method for rejecting background but are consistent with the double electron and double muon triggers that are 


\section{CHAPTER 4. HIGGS SEARCHES WITH ZZ DECAYS}

used. Muons are required to be well isolated from hadronic activity in the detector by restricting the sum of transverse momentum from the tracker or transverse energy in the ECal and HCal within a cone of $\Delta R=\sqrt{(\Delta \eta)^{2}+(\Delta \phi)^{2}}<0.3$ to be less than $15 \%$ of the measured $p_{T}$. Similar requirements are placed on electrons although the details depend also on the electron shower shape.

Reconstructed particle candidates are clustered with the anti- $k_{T}$ algorithm $[48,49]$ with a clustering parameter $R=0.5$. Jets are required to be in the tracker acceptance, $|\eta|<2.4$, to maximize the effectiveness of the PF algorithm. Energy corrections are applied to jets to account for systematic instrumental effects including the nonlinear energy response of the calorimeters. These corrections are derived from in-situ measurements [50]. Effects of pileup are mitigated by applying corrections according to the Fastjet algorithm [51]. Some requirement is also applied to the energy balance between the charged and neutral hadronic content in each jet. In some cases, jet substructure variables are used to distinguish on a statistical bases differences between gluon jets and quark jets. Gluon-like jets are removed from consideration. Finally, all jets are required to have $p_{T}>30 \mathrm{GeV}$.

With the basic objects in hand, the $2 \ell 2 q$ system is constructed under the assumption that all pairs of leptons and quarks are the daughters of $\mathrm{Z}$ bosons. Each di-lepton pair must have a combined invariant mass of $70<m_{\ell \ell}<110 \mathrm{GeV}$, thus reducing backgrounds which don't have an intermediate Z, like $t \bar{t}$ and QCD backgrounds. In order to reduce the overwhelming $\mathrm{Z}+$ jets background, the dijet invariant mass of the 

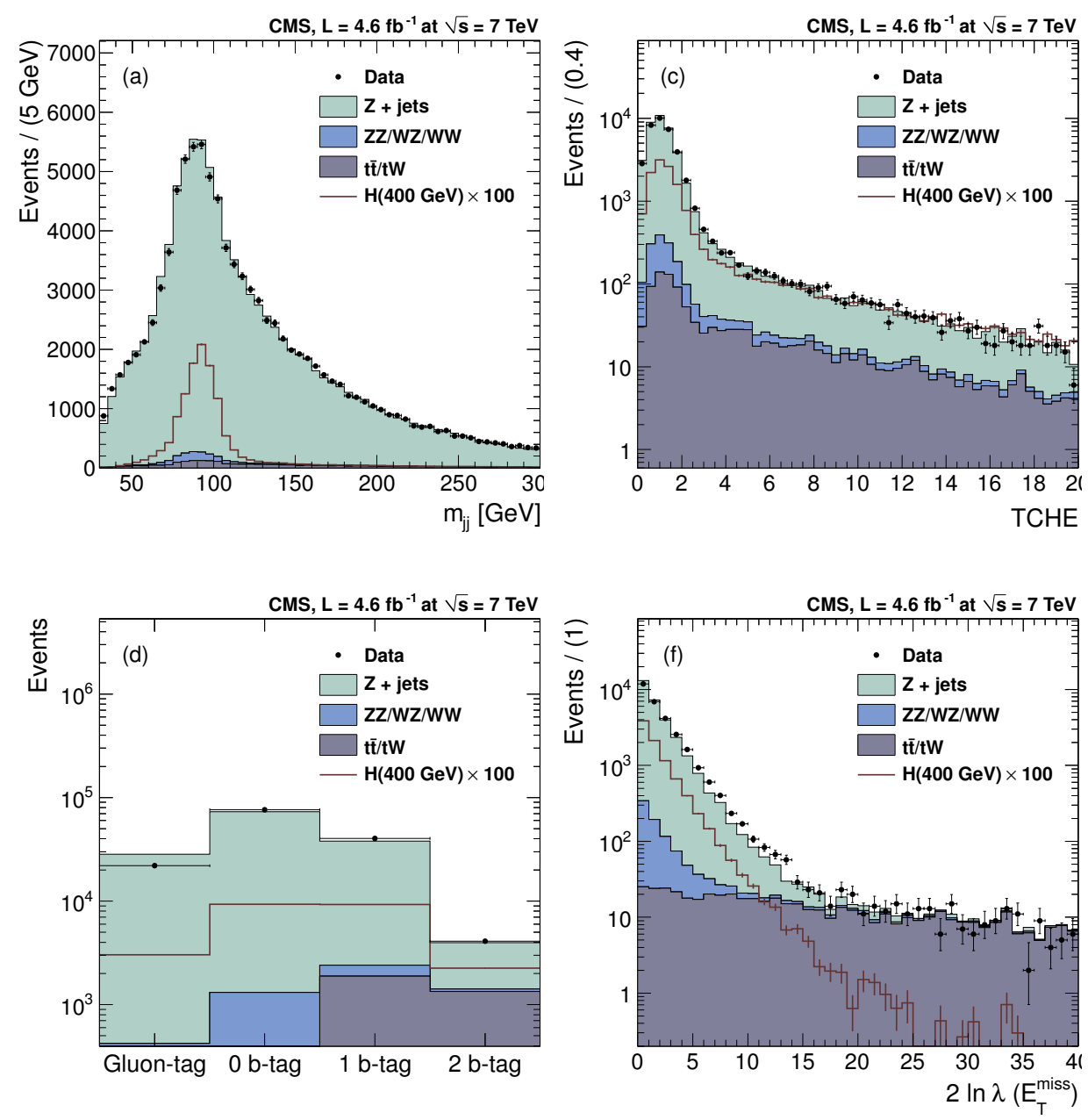

Figure 4.1: Distribution of $m_{j j}$ (top left), TCHE b-tagging discriminant (top right), and MET significance, $2 \ln \lambda\left(E_{T}^{\text {miss }}\right.$, (bottom left). Event category populations are shown in the bottom right plot. Filled histograms represent expectation of background events. Open, red histograms representation the expectation of a $400 \mathrm{GeV}$ Higgs boson whose cross section has been enhanced by $100 \times$. All events satisfy the preselection requirements. 


\section{CHAPTER 4. HIGGS SEARCHES WITH ZZ DECAYS}

event is required to satisfy $75<m_{j j}<105 \mathrm{GeV}$. Figure 4.1 shows the $m_{j j}$ for signal and background.

Categorizing events based on jet flavor provides a significant increase in sensitivity to signal events since b-jets are more likely to result from a Z decay than from QCD radiation in the $\mathrm{Z}+$ jets process. Furthermore, QCD radiation contains a large amount of gluon-jets, while the Z boson cannot decay into a pair of qluons. To isolate jets which are likely to originate from b-quarks, the CMS track counting high-efficiency (TCHE) b-tagging algorithm $[52,53]$ is used. This algorithm relies on tracks within the jet cone having large impact parameters, indicating a displaced vertex. This information is encompassed in a discriminant which is used to determine how b-like jets and is shown in figure 4.1. Using this discriminant, the events are divided into three categories: those which have at least one jet passing the median working point $\left(\sim 65 \%\right.$ efficient $\left.^{1}\right)$ and another jet passing the loose working point $(\sim 80 \%$ efficient $)$; those which have at least one jet passing the loose working point; those which have zero jets passing the loose working point. Although there is a non-negligible mistag rate for each of these working points, the categories are referred to as 2 b-tag, 1 btag, and 0 b-tag, respectively. The categories are defined such that they are mutually exclusive by putting events in the category with the most stringent requirements. Gluon-like jets are removed from the 0 b-tag category. The division of events in each of the three categories is shown if figure 4.1.

\footnotetext{
${ }^{1}$ More information on b-tagging efficiencies and mistagging rates can be found elsewhere $[52,53]$
} 


\section{CHAPTER 4. HIGGS SEARCHES WITH ZZ DECAYS}

Since there is a significant amount of $t \bar{t}$ and $t W$ events in the 2 b-tag category, events in which the PF candidate collection has a significant imbalance of transverse energy, also known as missing transverse energy, $E_{T}^{\text {miss }}$, are removed. To quantify this,

a likelihood ratio, $\lambda\left(E_{T}^{\text {miss }}\right)$, is built comparing two hypothesis, $E_{T}^{\text {miss }}=0$ and $E_{T}^{\text {miss }} \neq$ 0 [54]. Events in the 2 b-tag category are then required to satisfy $2 \ln \lambda\left(E_{T}^{\text {miss }}\right)<10$. In the low mass analysis, we instead require $E_{T}^{\text {miss }}<50 \mathrm{GeV}$ in the 2 b-tag category.

\section{MELA Discriminant}

In order to further reduce the overwhelming background, $\mathrm{Z}+$ jets, in the high mass analysis, the MELA technique is employed. The five angular variables described in chapter 3 are used. Above threshold, the $\mathrm{Z}$ masses provide little discrimination power and are dropped for simplicity. The discriminate makes use of the 5D probability distributions, $\mathscr{P}\left(\cos \theta^{*}, \cos \theta_{1}, \cos \theta_{2}, \Phi, \Phi_{1} \mid m_{Z Z}\right)$, according to

$$
D=\frac{\mathscr{P}_{\text {Higgs }}}{\mathscr{P}_{\text {Higgs }}+\mathscr{P}_{\text {Zjets }}} .
$$

The expected and observed distributions for these 5 angles for both signal and background are shown in figure 4.5.

Since the ideal distributions for the dominant background cannot be described analytically in terms of the angular variables, the distributions are found empirically from MC, including all detector effects, assuming no correlations between the 5 angular variables. The $\cos \theta_{1}, \cos \theta_{2}$, and $\cos \theta^{*}$ projections are modeled with even 


\section{CHAPTER 4. HIGGS SEARCHES WITH ZZ DECAYS}

polynomials in the corresponding variable. This makes use of prior knowledge that the distributions should be symmetric. In addition, the $\cos \theta_{2}$ projection includes a Fermi-Dirac distribution to model the sharp acceptance effect found with the hadronic $\mathrm{Z}$ near $\cos \theta_{2}=1$. In general, acceptance effects arise near $\cos \theta_{1,2}=1$ from pseudorapidity cuts. In this, case, $\cos \theta_{2}$ describes the angular distributions of the jets in the rest frame of its parent $\mathrm{Z}$ and the finite extent of the jet enhances the acceptance effect. The $\Phi$ and $\Phi_{1}$ projections are modeled with a finite Fourier series. Fits to $\mathrm{Z}+$ jets $\mathrm{MC}$ are performed in slices of $m_{Z Z}$. The parameters of these fits are then interpolated slices so that $\mathscr{P}_{Z \text { jets }}$ is continuous in $m_{Z Z}$. Some examples of these fits are shown in figure 4.2 .

The signal parametrization must also include detector effects. The ideal distributions from section 3 are modified with 5D uncorrelated function which is then fit to MC to account for any detector effects. The parametrization of detector effects is the same as those used for describing background. Also as with background, these fits are performed in slices of $m_{Z Z}$ and extrapolated to arbitrary values. Examples of the signal parametrization are shown in figure 4.3.

Combining these two density functions together, the discriminant, D, is shown in figure 4.5. The signal events tend to peak more towards 1 while the background events tend to peak more towards zero. This variable is then used to select signallike events. Because the shape of D changes with $m_{Z Z}$, the optimal cut will be $m_{Z Z}$

dependent. An optimization was run using $\kappa=N_{s i g} / \sqrt{N_{b k g}}$ as a figure of merit. This 


\section{CHAPTER 4. HIGGS SEARCHES WITH ZZ DECAYS}

variable represents an approximation of the expected upper limit, UL. For a simple counting experiment in which the expected number of background events is large, $\kappa$ is a good approximation of the true UL. The optimization was performed separately for each of the three b-tagging categories and the proposed D cuts along with the cuts used for other variables are shown in table 4.2.

The angular variables which are used as input to the angular D represent a set of variables which are only loosely correlated with the final discriminating variable, $m_{Z Z}$. As a result, cutting on this variables does not significantly alter the shape of the $m_{Z Z}$ distribution. In contrast, an optimized set of cuts on more traditional variables $\left(p_{T, l e p t o n}, p_{T, j e t}, p_{T, \ell \ell}, \Delta R_{\text {jets }}\right)$, which are highly correlated with $m_{Z Z}$, would produce a peak for background as well as signal. This is demonstrated in figure 4.4, where an optimized cut on both sets of variables is applied and the resulting $m_{Z Z}$ is shown. The preservation of the $m_{Z Z}$ shape allows for the expected background distribution to be easily described through simple analytical functions which can then be used for and used for statistical interpretation of the final observed distributions.

\subsubsection{Yields and Kinematics Distributions}

From figures 4.1 and 4.5 it is clear that the agreement between data and MC is fairly good. Although there are some disagreements in some of the distributions, these disagreements reflect the complexity that exists in modeling inclusive $\mathrm{Z}$ production. To ensure that background estimations are reliable in the more restricted phase space 


\section{CHAPTER 4. HIGGS SEARCHES WITH ZZ DECAYS}
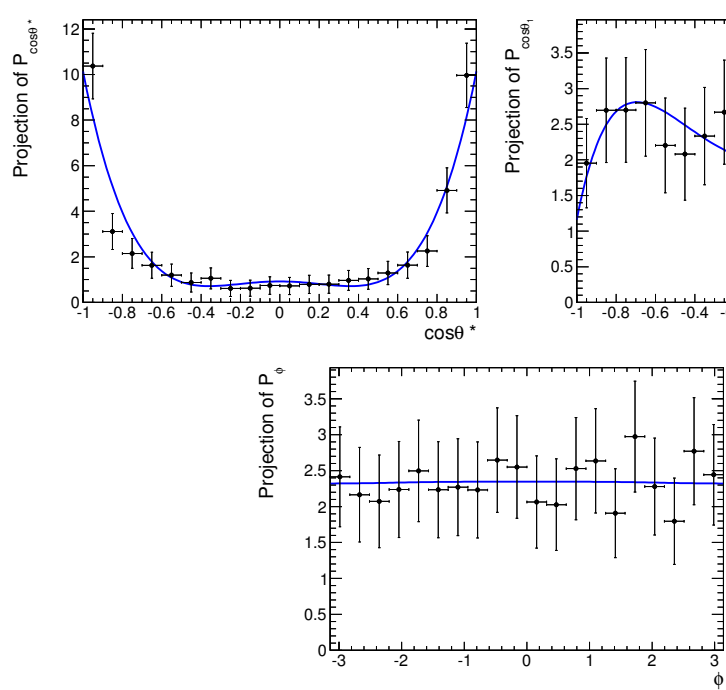
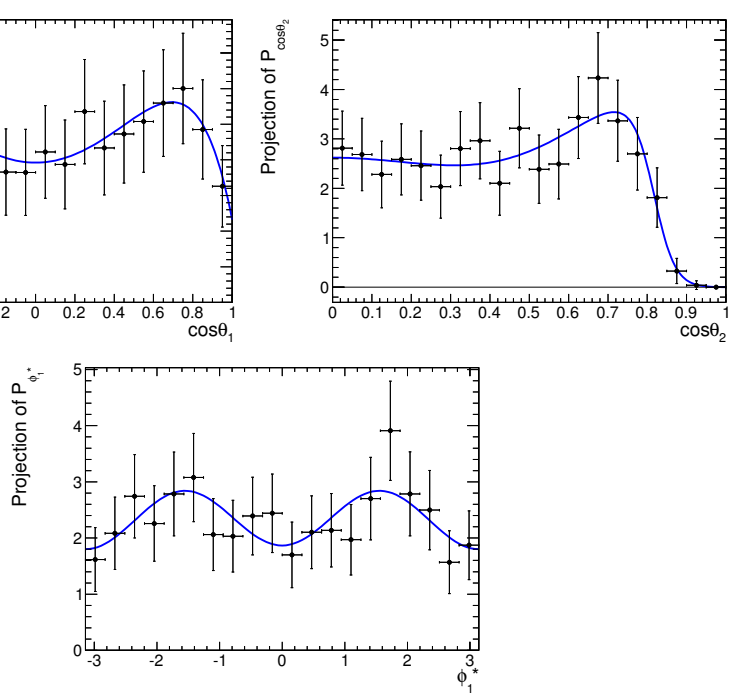

Figure 4.2: Empirical derivation of 5D PDF for $\mathrm{Z}+$ jets events. Points represent expected distributions of events between $475<m_{Z Z}<550 \mathrm{GeV}$ from MC simulation, lines represent the final model at the median $m_{Z Z}$ value.
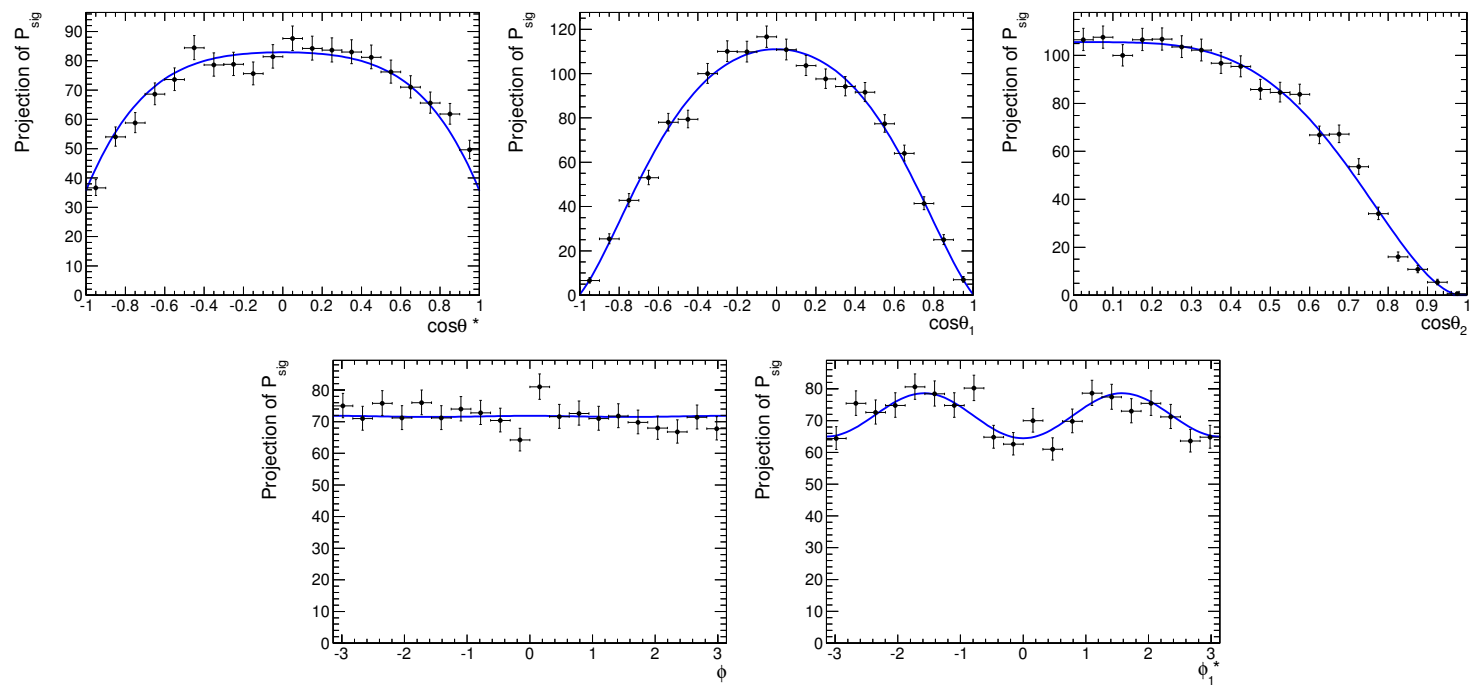

Figure 4.3: Empirical derivation of 5D PDF for signal events. Points represent expected distributions of events for $m_{H}=500 \mathrm{GeV}$ from MC simulations, lines represent the final model at the median $m_{Z Z}$ value. 


\section{CHAPTER 4. HIGGS SEARCHES WITH ZZ DECAYS}
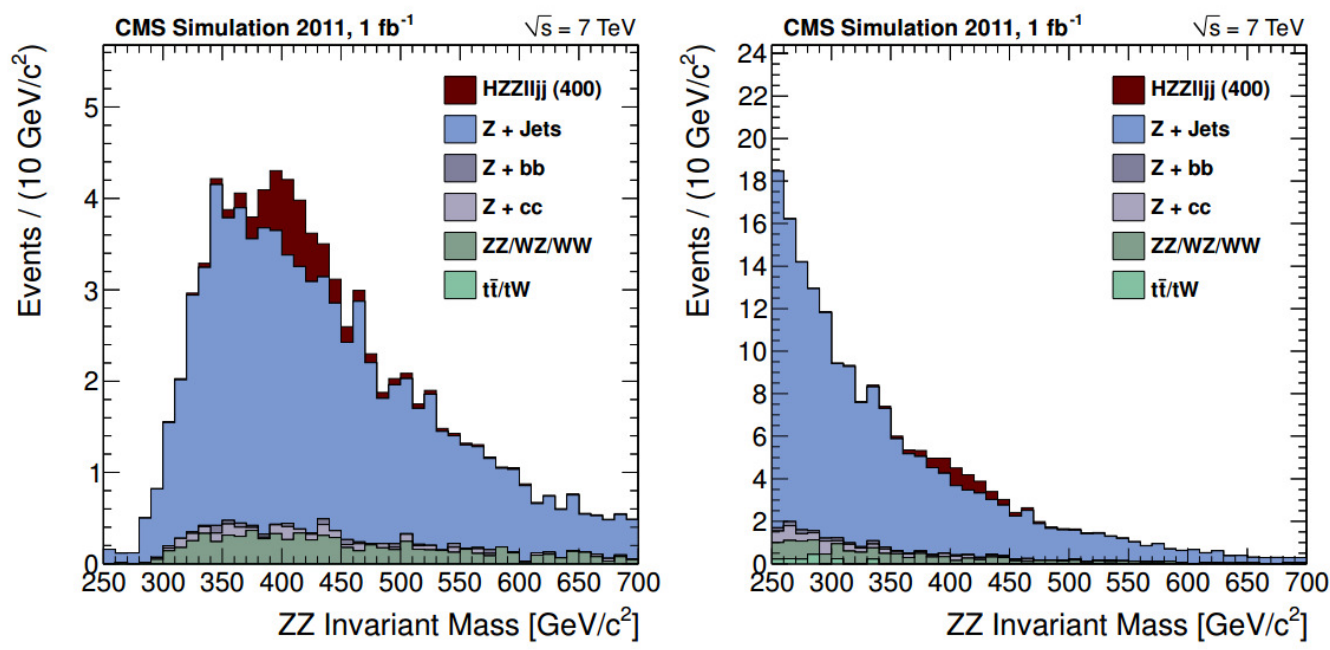

Figure 4.4: Distribution of $m_{Z Z}$ after optimal cut on angular D (right) and traditional variables, (left). Maroon histogram represents expected distribution of a $400 \mathrm{GeV}$ SM Higgs, blue and green histograms represent different SM backgrounds from MC simulations.

\begin{tabular}{|c|c|c|c|}
\hline \multicolumn{4}{|c|}{ preselection } \\
\hline $\begin{array}{c}p_{T}\left(\ell^{ \pm}\right) \\
p_{T}(\text { jets }) \\
|\eta|\left(\ell^{ \pm}\right) \\
|\eta|(\text { jets })\end{array}$ & \multicolumn{3}{|c|}{ 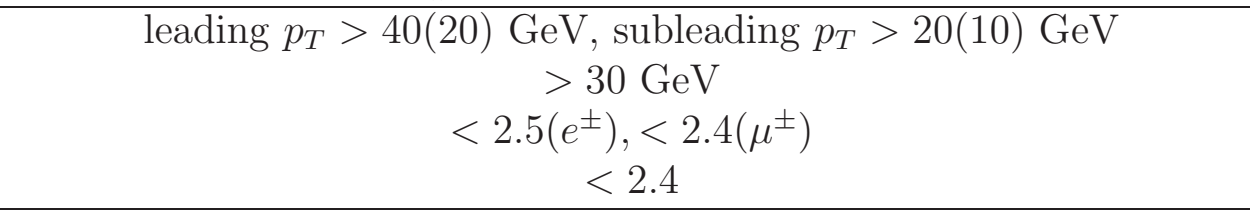 } \\
\hline \multicolumn{4}{|c|}{$\begin{array}{lc}\text { final selection } \\
\end{array}$} \\
\hline & $0 \mathrm{~b}-\mathrm{tag}$ & $1 \mathrm{~b}-\mathrm{tag}$ & $2 \mathrm{~b}-\mathrm{tag}$ \\
\hline $\begin{array}{l}\mathrm{b}-\mathrm{tag} \\
\mathrm{D} \\
E_{T}^{m i s s}\end{array}$ & $\begin{array}{c}\text { none } \\
>0.55+0.00025 m_{Z Z} \\
\text { none }\end{array}$ & $\begin{array}{c}1 \text { loose } \\
>0.302+0.000656 m_{Z Z} \\
\text { none }\end{array}$ & $\begin{array}{l}1 \text { loose } \& 1 \text { medium } \\
\quad>0.5 \\
2 \ln \lambda\left(E_{T}^{\text {miss }}\right)<10 \\
\left(E_{T}^{\text {miss }}<50 \mathrm{GeV}\right)\end{array}$ \\
\hline $\begin{array}{l}m_{j j} \\
m_{\ell \ell} \\
m_{Z Z}\end{array}$ & \multicolumn{3}{|c|}{$\begin{array}{c}\in[70,110](<80) \mathrm{GeV} \\
\in[183,800](\in[125,170]) \mathrm{GeV}\end{array}$} \\
\hline
\end{tabular}

Table 4.2: Table listing analysis selections. The top portion details preselection cuts applied to all objects to be consistent with trigger requirements and detector acceptance. The bottom portion details all cuts applied in each of the different b-tag categories to optimize the sensitivity to signal events. 


\section{CHAPTER 4. HIGGS SEARCHES WITH ZZ DECAYS}
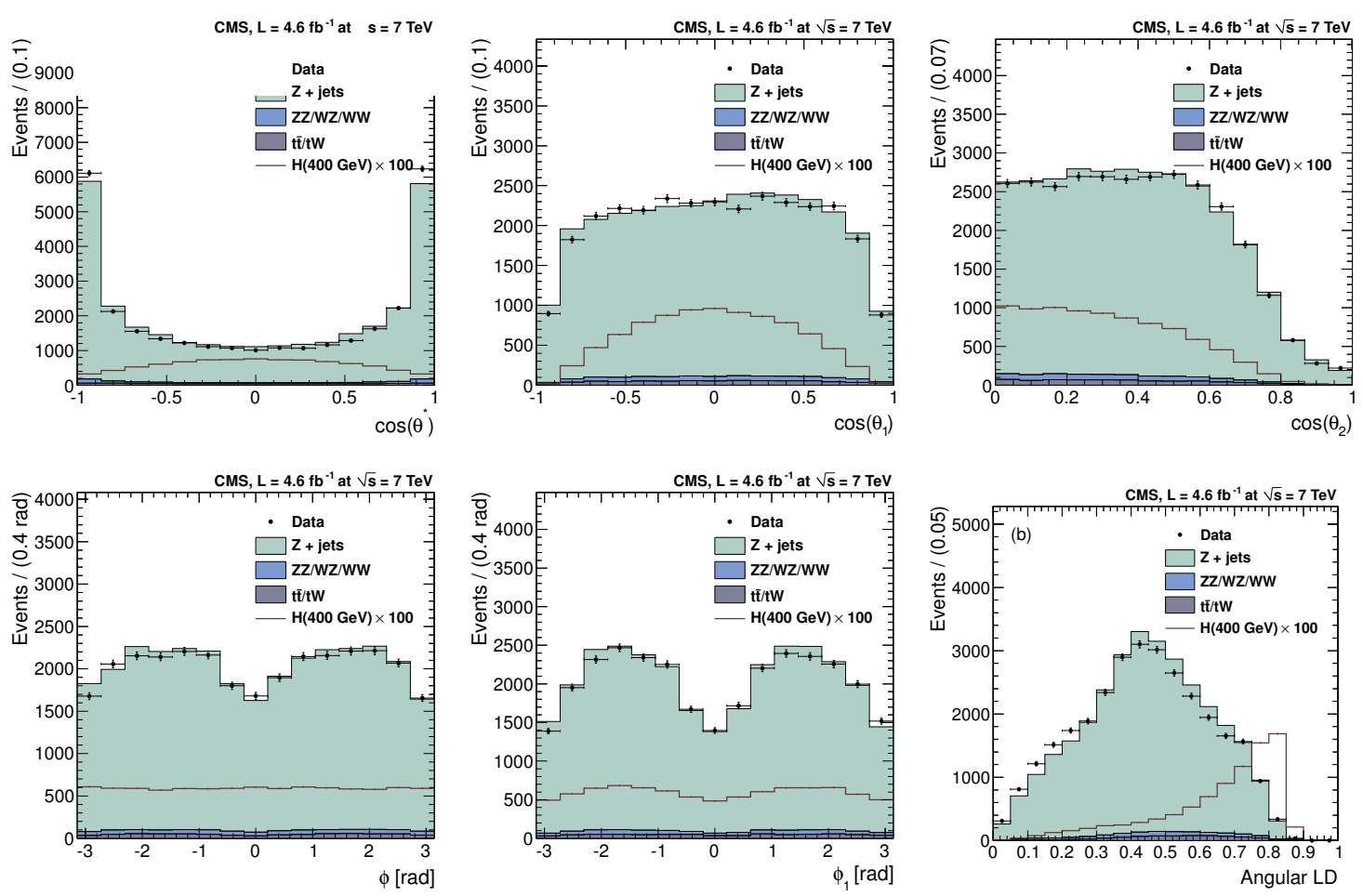

Figure 4.5: Distribution of 5 angles used to build the angular likelihood discriminant, shown in the bottom right plot. Filled histograms represent expectation of background events. Open, red histograms representation the expectation of a $400 \mathrm{GeV}$ Higgs boson whose cross section has been enhanced by $100 \times$. All events satisfy the preselection requirements. 


\section{CHAPTER 4. HIGGS SEARCHES WITH ZZ DECAYS}

of the final selections, it is important to have a methodology for measuring background shapes and normalizations directly from data.

Data control regions are defined using events passing all of the final selections in table 4.2 but instead lie in the regions $60<m_{j j}<75 \mathrm{GeV}$ or $105<m_{j j}<130 \mathrm{GeV}$. These regions are mutually exclusive from the signal region, $75<m_{j j}<105 \mathrm{GeV}$, and include only a small contribution from signal events, as evident from figure 4.1. Since the kinematics of this control region are not expected to be exactly the same as the signal region, events are reweighted to account for the differences between the signal region and the control region. The expected number of background events in a given $m_{Z Z}$ range can be estimated by

$$
N_{b k g}\left(m_{Z Z}\right)=N_{C R}\left(m_{Z Z}\right) \times \frac{N_{b k g}^{s i m}\left(m_{Z Z}\right)}{N_{C R}^{s i m}\left(m_{Z Z}\right)}=N_{C R}\left(m_{Z Z}\right) \times \alpha\left(m_{Z Z}\right),
$$

where $N_{b k g}$ is the number of events expected in data in the signal region, $N_{C R}$ is the number of events observed in the data control region, and $N_{C R}^{s i m}, N_{b k g}^{s i m}$ are the events measured in the MC control region and signal region, respectively. Thus, $\alpha$ represents the weight for extrapolating between the signal and control region and is calculated using MC simulation. These weights range between 0.75 and 1.2 and have been calculated with two different MC generators, MADGRAPH and SHERPA, both give statistically compatible results. Both the expected shape and normalization of the SM background are calculated with this method for each o the three b-tag categories 


\section{CHAPTER 4. HIGGS SEARCHES WITH ZZ DECAYS}

separately.

Once the expected distributions are calculated, the shape of the background is fit using an empirical function. A crystal ball ${ }^{2}$ function multiplied by a Fermi-Dirac distribution was found to provide a good description of the background in the three different b-tag categories in MC. The uncertainties of the fit parameters and the statistical uncertainties on $\alpha$ are taken as systematic uncertainties in the background estimation for the final statistical analysis. Figure 4.6 shows the expected shape and normalization of the $m_{Z Z}$ distribution taken directly from MC (filled histograms), the data-driven estimation of the background shape and normalization (blue line), and the observed distribution from data (points with error bars). Although the MC generally does a reasonably good job of describing the observed distribution, there are some minor systematic effects which are corrected for by the data-driven estimation. The SM Higgs expectation enhanced by a factor 2 (5) or a Higgs mass of 400 (150) GeV is also shown in yellow.

While the background shapes and event yields are derived from data, the signal model is derived from MC simulations. Signal production cross sections and branching ratios are taken from the LHC Higgs Cross Sections Working Group and others [33, 55, 55-75] production cross sections are calculated at NNLO. Signal efficiencies are taken from CMS simulations and are corrected for known differences between data and MC using tag and probe measurements. The efficiencies are also interpolated

\footnotetext{
${ }^{2}$ A crystal ball function is a piece-wise function which incorporates a Gaussian core with a power law tail. These functions are connected in such a way that the function is continuous and smooth.
} 


\section{CHAPTER 4. HIGGS SEARCHES WITH ZZ DECAYS}
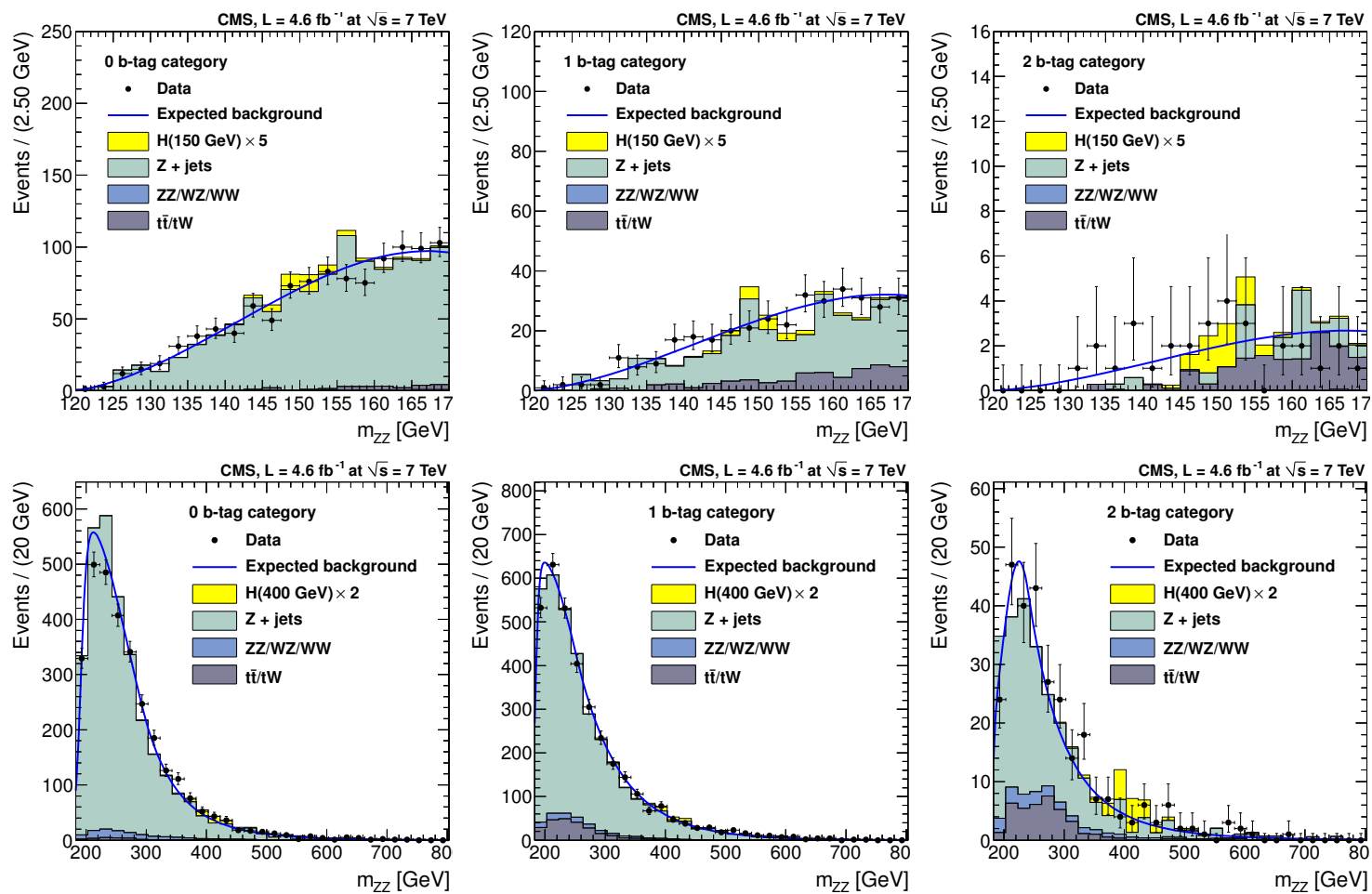

Figure 4.6: The $m_{Z Z}$ invariant mass distribution after final selection in three categories: 0 b-tag (top), 1 b-tag (middle), and 2 b-tag (bottom). The low-mass range, $120<m_{Z Z}<170 \mathrm{GeV}$ is shown on the left and the high-mass range, $183<m_{Z Z}<800 \mathrm{GeV}$ is shown on the right. Points with error bars show distributions of data and solid curved lines show the prediction of background from the control region extrapolation procedure. In the low-mass range, the background is estimated from the $m_{Z Z}$ for each Higgs mass hypothesis and the average expectation is shown. Solid histograms depicting the background expectation from simulated events for the different components are shown. Also shown is the SM Higgs boson signal with the mass of 150 (400) GeV and cross section 5 (2) times that of the SM Higgs boson, which roughly corresponds to the expected exclusion limits in each category. 


\section{CHAPTER 4. HIGGS SEARCHES WITH ZZ DECAYS}

to intermediate values of $m_{H}$ using a polynomial fit. Figures 4.8 and 4.9 shows the efficiency curves for each of the 6 categories. The efficiencies together with the production cross section and branching ratio are used to derive the expected event yields.

Signal shapes are modeled using both POWHEG to model the production of Higgs bosons at NLO in $\alpha_{s}$ and PYTHIA to model the decay kinematics. In order to get a good description of the signal shape, events are fit in two separate categories. Those in which both the jets used to build the $\mathrm{Z}$ are matched to generator level quarks from the Higgs decay, and those in which the jets are not matched. The latter category represents event in which the Higgs was mis-reconstructed and thus is expected to have a much broader distribution. Matched events are fit with a double crystal ball function (i.e. a Gaussian distribution whose tails are described by two independent power law distributions). Unmatched events are fit with a triangle function convoluted with a crystal ball function. Signal samples corresponding to different mass hypotheses, $m_{H}$, are fit separately and the shape parameters are then interpolated for intermediate mass hypotheses. This procedure is performed separately for each b-tag category. Examples of the signal shape model are shown for a $130 \mathrm{GeV}$ and $400 \mathrm{GeV}$ Higgs boson for each of the three b-tag categories separately in figure 4.7.

A number of systematic uncertainties are associated with the calculation of the number of expected event yields. Many of these result from limited understanding of reconstruction efficiencies. The muon and electron reconstruction efficiencies have 


\section{CHAPTER 4. HIGGS SEARCHES WITH ZZ DECAYS}
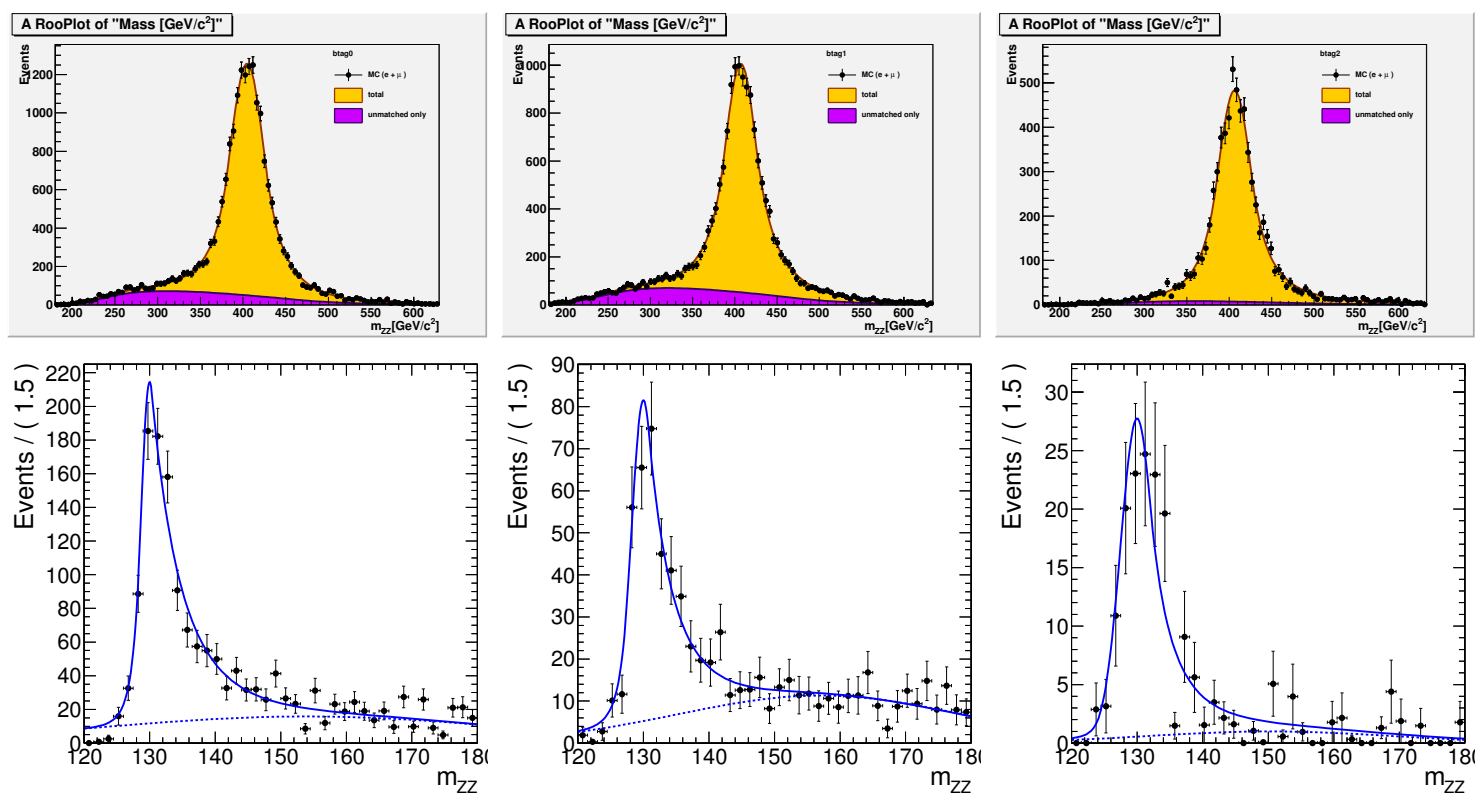

Figure 4.7: Signal shapes models for $400 \mathrm{GeV}$ (top row) and $130 \mathrm{GeV}$ (bottom row) signals for each of the three b-tag categories, 0 b-tag (left), 1 b-tag (middle), and 2 b-tag (right).

been assigned uncertainties of $2.7 \%, 4.5 \%$, respectively. Jet efficiency uncertainties due to JES range from 1-8\% depending on the Higgs mass hypothesis. The efficiency uncertainty of $E_{T}^{\text {miss }}$ cuts range from $3-4 \%$. The b-tagging efficiency uncertainties depend both on the category as well as the Higgs mass hypothesis and range between $2-11 \%$. The additional jet identification requirements applied in the 0 b-tag category, including gluon-tagging, is assigned an uncertainty of $4.6 \%$. Uncertainties from Higgs production, either through parton distribution functions, missing higher order corrections, or VBF modeling are assigned to both the overall cross section calculation or the effect on acceptance due to shape differences. Theoretical uncertainties on signal shapes introduce some additional systematic to the effective amount of event near 


\section{CHAPTER 4. HIGGS SEARCHES WITH ZZ DECAYS}
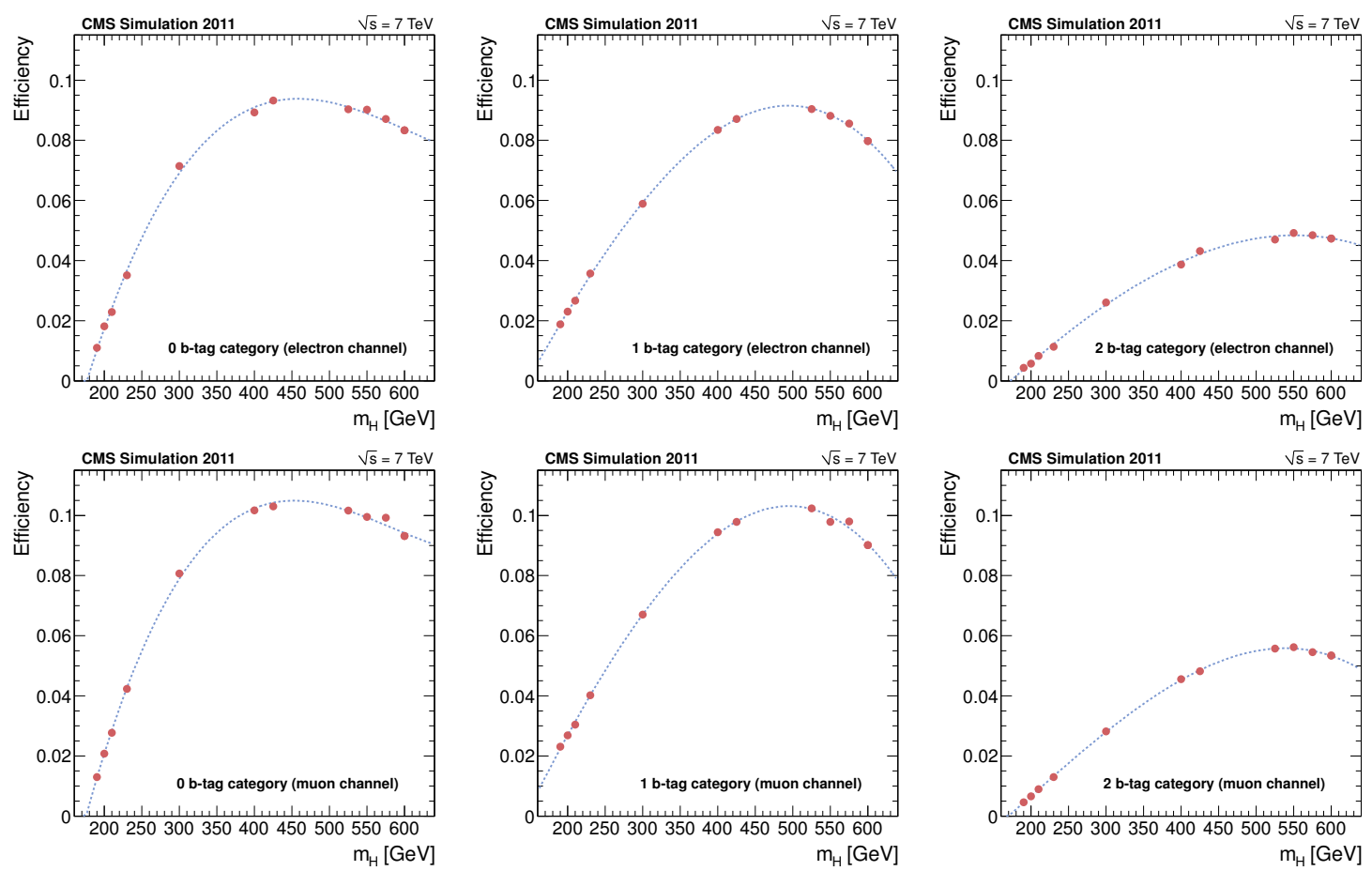

Figure 4.8: Signal efficiency parametrization in each of the 6 different categories of the high mass signal samples. 


\section{CHAPTER 4. HIGGS SEARCHES WITH ZZ DECAYS}
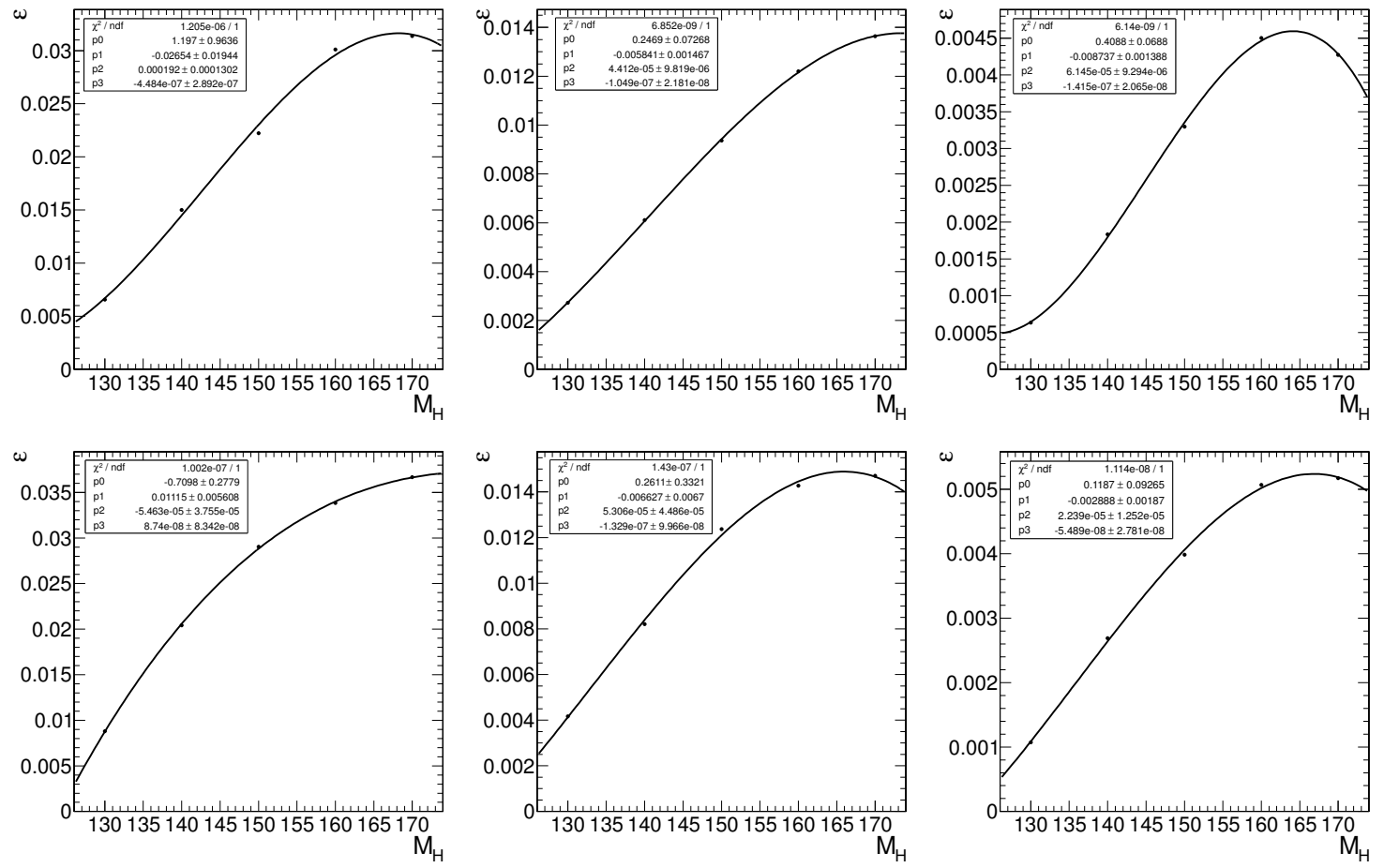

Figure 4.9: Signal efficiency parametrization in each of the 6 different categories of the low mass signal samples. 


\section{CHAPTER 4. HIGGS SEARCHES WITH ZZ DECAYS}

\begin{tabular}{|c|c|c|c|}
\hline source & $0 \mathrm{~b}-\mathrm{tag}$ & $1 \mathrm{~b}-\mathrm{tag}$ & $2 \mathrm{~b}-\mathrm{tag}$ \\
\hline muon reconstruction & \multicolumn{3}{|c|}{$2.7 \%$} \\
\hline electron reconstruction & \multicolumn{3}{|c|}{$4.5 \%$} \\
\hline jet reconstruction & \multicolumn{3}{|c|}{$1-8 \%$} \\
\hline pile-up & \multicolumn{3}{|c|}{$3-4 \%$} \\
\hline$E_{T}^{\text {miss }}$ & - & - & $3-4 \%$ \\
\hline b-tagging & $2-7 \%$ & $3-5 \%$ & $10-11 \%$ \\
\hline gluon-tagging & $4.6 \%$ & - & - \\
\hline acceptance $(\mathrm{HqT})$ & $2 \%$ & $5 \%$ & $3 \%$ \\
\hline acceptance(PDF) & \multicolumn{3}{|c|}{$3 \%$} \\
\hline acceptance(VBF) & \multicolumn{3}{|c|}{$1 \%$} \\
\hline signal cross section (PDF) & \multicolumn{3}{|c|}{$8-10 \%$} \\
\hline signal cross section (scale) & \multicolumn{3}{|c|}{$8-11 \%$} \\
\hline signal shape & \multicolumn{3}{|c|}{$1.5 \times 10^{-7 \%} \% \times m_{H}^{3}[\mathrm{GeV}]$} \\
\hline luminosity & \multicolumn{3}{|c|}{$4.5 \%$} \\
\hline
\end{tabular}

Table 4.3: Summary of systematic uncertainties on signal normalization. Most sources give multiplicative uncertainties on the cross section measurement, except for the expected Higgs boson production cross section, which is relevant for the measurement of the ratio to the SM expectation. The ranges indicate dependence on $m_{H}$.

the signal peak. Since the width depends strong on the mass hypothesis, $m_{H}$, the uncertainties also depends on $m_{H}$ according to $1.5 \times 10^{-7 \%} \times m_{H}^{3}[\mathrm{GeV}]$. Finally, uncertainties from luminosity measurements are accounted for in the signal systematics. All systematic uncertainties on the signal yields are summarized in table 4.3.

\subsubsection{Results of Semilepton Analysis}

The expected background event yields, both from MC simulation and from the data-driven estimations, and expected signal event yields are compared against the observed event yields in each of the three b-tag categories in table 4.4. Since there 


\section{CHAPTER 4. HIGGS SEARCHES WITH ZZ DECAYS}

\begin{tabular}{l|c|c|c}
\hline \hline & 0 b-tag & 1 b-tag & 2 b-tag \\
\hline \hline \multicolumn{4}{c}{$m_{Z Z} \in[125,170]$} \\
\hline observed yield & 1087 & 360 & 30 \\
expected background (data-driven) & $1050 \pm 54$ & $324 \pm 28$ & $19 \pm 5$ \\
expected background (MC) & $1089 \pm 39$ & $313 \pm 20$ & $24 \pm 4$ \\
\hline \hline \multicolumn{4}{c}{$m_{Z Z} \in[183,800]$} \\
\hline observed yield & 3036 & 3454 & 285 \\
expected background (data-driven) & $3041 \pm 54$ & $3470 \pm 59$ & $258 \pm 17$ \\
expected background (MC) & $3105 \pm 39$ & $3420 \pm 41$ & $255 \pm 11$ \\
\hline \hline \multicolumn{4}{c}{ signal expectation (MC) } \\
\hline$m_{H}=150 \mathrm{GeV}$ & $10.1 \pm 1.5$ & $4.1 \pm 0.6$ & $1.6 \pm 0.3$ \\
\hline$m_{H}=250 \mathrm{GeV}$ & $24.5 \pm 3.5$ & $21.7 \pm 3.0$ & $8.1 \pm 1.7$ \\
\hline$m_{H}=350 \mathrm{GeV}$ & $29.6 \pm 4.3$ & $26.0 \pm 3.7$ & $11.8 \pm 2.5$ \\
\hline$m_{H}=450 \mathrm{GeV}$ & $16.5 \pm 2.4$ & $15.8 \pm 2.2$ & $7.9 \pm 1.7$ \\
\hline$m_{H}=550 \mathrm{GeV}$ & $6.5 \pm 1.0$ & $6.5 \pm 0.9$ & $3.6 \pm 0.8$ \\
\hline \hline
\end{tabular}

Table 4.4: Observed and expected event yields for $4.6 \mathrm{fb}^{-1}$ of data. The yields are quoted in the ranges $125<m_{Z Z}<170 \mathrm{GeV}$ or $183<m_{Z Z}<800 \mathrm{GeV}$, depending on the Higgs boson hypothesis. The expected background is quoted from both the data-driven estimations and from MC simulations directly. In the low-mass range, the background is estimated from the $m_{Z Z}$ sideband for each Higgs mass hypothesis and is not quoted in the table. The errors on the expected background from simulation include only statistical uncertainties.

are no significant excesses found in any of the observed invariant mass spectra, limits on the Higgs cross section are calculated.

A simultaneous fit of the $m_{Z Z}$ distributions for the signal cross section in the six different channels is perform using a dedicated statistical software package discussed in ref. [76]. Using the distribution of the $C L_{S}$ test statistic [77], 95\% confidence level (CL) limits are calculated. Expected limits are derived from pseudoexperiments which are generated based on expected distributions. Nuisance parameters associated with the different systematic uncertainties are randomized when generating toys and 

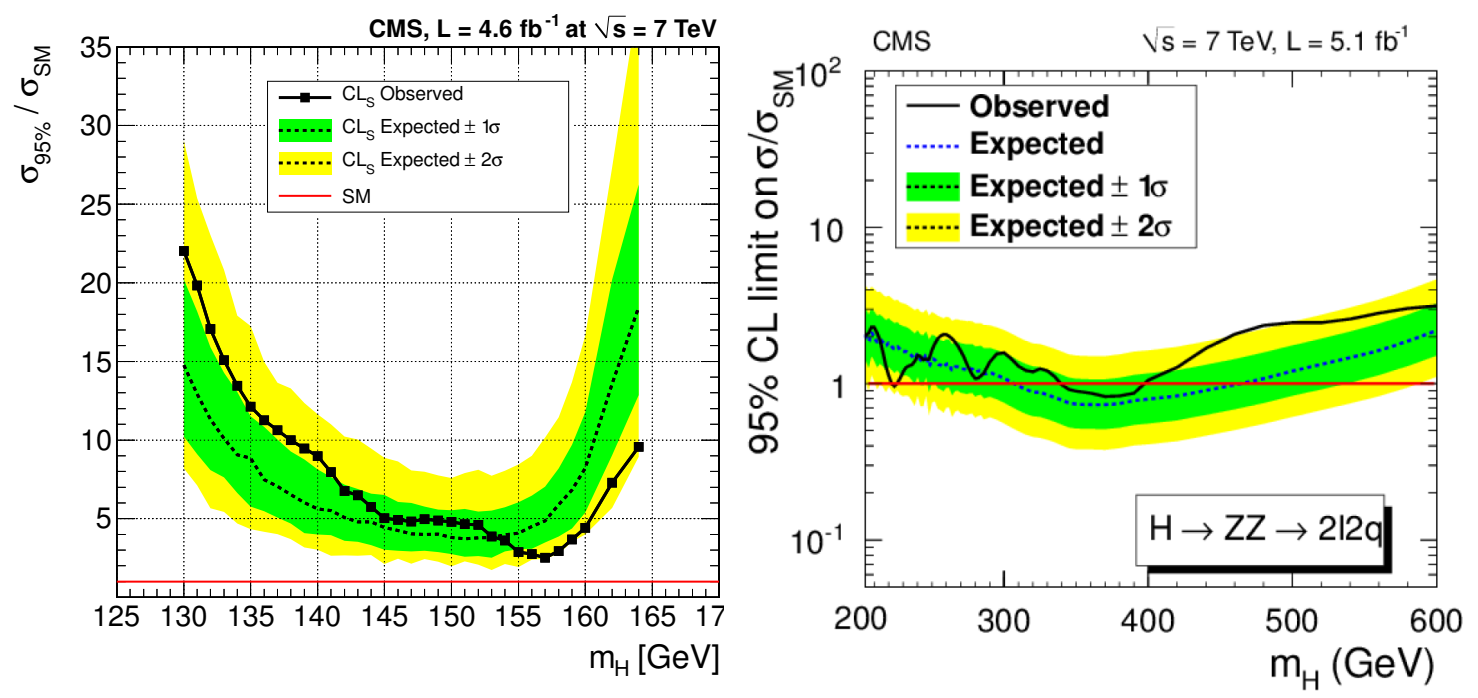

Figure 4.10: Observed (solid) and expected (dashed) 95\% CL upper limit on the ration $\mathrm{f}$ the production cross section o the SM expectation for the Higgs boson obtained using the $C L_{s}$ technique. The $68 \%(1 \sigma)$ and $95 \%(1 \sigma)$ ranges of expectation for the background-only model are shown with green and yellow bands, respectively. The solid line at 1 indicates the SM expectation. Left: low-mass range, right: high-mass range.

profiled in fits.

The expected and observed distributions of the 95\% CL upper limit on the ratio of the observed cross section with respect to the Higgs cross section, $\sigma_{95 \%} / \sigma_{S M}$, is shown figure 4.10. While the low mass region limits are at best around several times SM Higgs cross sections, the high mass region has an expected exclusion for Higgs masses in the range $[310,460] \mathrm{GeV}$. The observed data excludes Higgs boson masses in the range $[340,390] \mathrm{GeV}$. 


\section{CHAPTER 4. HIGGS SEARCHES WITH ZZ DECAYS}

\subsection{Golden Decay Channel}

The $Z Z \rightarrow 4 \ell$ channel, often referred to as the golden decay channel, is one of the most promising channels for discovering a Higgs like resonance over a broad range of masses because of the high mass resolution and low SM background rates. Using the tools developed in Chapter 3, it will be shown that this channel is also very conducive for property measurements of resonances.

\subsubsection{Datasets}

Events used are selected either via the double electron, double muon, or triple electron triggers. The double electron and muon triggers require that the transverse momentum, $p_{T}$, of the leading and sub-leading leptons be greater than 17 and $8 \mathrm{GeV}$, respectively; the triple electron triggers thresholds are 15,8 , and $5 \mathrm{GeV}$, respectively. The efficiencies for these triggers are found to be at least $98 \%$ for a SM Higgs boson with $m_{H}>120 \mathrm{GeV}$.

Monte Carlo (MC) simulations have been used to develop, optimize, and validate analysis strategies. Signal samples are generated using either POWHEG [39] at next-toleading order (NLO) in $\alpha_{s}$ for SM Higgs samples via gluon-gluon fusion or VBF. For SM Higgs and non-SM signals samples at leading order, JHUGen [1,35]. For simulation of Higgs bosons produced in association with either weak vector bosons, VH, or $t \bar{t}$ pairs, ttH, the event generator PYTHIA [43] is used. 


\section{CHAPTER 4. HIGGS SEARCHES WITH ZZ DECAYS}

Since the PYTHIA samples do not model the interference of final leptons for the $4 \mu$ and $4 e$ channels. These samples are reweighted using the JHUGen matrix element calculation where appropriate. However, the branching fractions $\mathscr{B}(H \rightarrow 4 \ell)$ are taken from PROPHECY4F which includes both interference effects and NLO QCD/EW corrections. The narrow-width approximation for the $m_{4 \ell}$ line shape is employed at low mass resulting in a Breit-Wigner distribution. At larger masses were the Higgs width become large, the $m_{4 \ell}$ line shape is reweighted to match the complex-pole scheme described in [78-80]. Effects from the interference between signal and the continuum $g g \rightarrow Z Z$ production is also accounted for following the prescription of [81]. The total production cross section of the Higgs boson is taken from References [33, 55-65] for gluon-gluon fusion process and according to References [33,67-71] for VBF process.

The SM continuum production of $\mathrm{ZZ}$ events via $q \bar{q}$ annihilation is simulated at NLO using POWHEG while other diboson processes were simulated with MADGRAPH [41]. The gluon-gluon fusion production of continuum ZZ events is simulated using GG2ZZ [82]. Drell-Yan events are simulated at LO using MADGRAPH. Di-boson samples produced at leading order are rescaled to match cross sections predicted by NLO calculations while Drell-Yan samples are rescaled to match cross sections predicted by NNLO calculations. Finally $t \bar{t}$ events are simulated at NLO with POWHEG. The generators and cross sections for each of these event types is shown in Table 4.5

All initial-state and final-state radiation is modeled using PYTHIA. Parton density function are taken from CTEQ6L [83] (CT10 [45]) for LO (NLO) generators. Detector 
CHAPTER 4. HIGGS SEARCHES WITH ZZ DECAYS

\begin{tabular}{c|c}
\hline \hline Sample Name & Generator \\
\hline$p p \rightarrow H \rightarrow Z Z^{(*)} \rightarrow 4 \ell$ & POWHEG \\
$g g \rightarrow H \rightarrow Z Z^{(*)} \rightarrow 4 \ell$ & JHUGen \\
$X \rightarrow Z Z^{(*)} \rightarrow 4 \ell$ & JHUGen \\
$\mathrm{Z}+\mathrm{X}$ & MADGRAPH \\
$t \bar{t}$ & POWHEG \\
WW\&ZW & MADGRAPH \\
$q \bar{q} \rightarrow Z Z$ & POWHEG \\
$g g \rightarrow Z Z$ & GG2ZZ \\
\hline \hline
\end{tabular}

Table 4.5: List of MC samples used for the $Z Z^{(*)} \rightarrow 4 \ell$ analysis. along with the event generator used to simulate them.

effects and event reconstruction is simulated using GEANT4 [84]. The number of reconstructed vertices per collision is reweighted to match the distribution seen in data. Additional energy deposited into calorimeter from pileup interactions and from the underlying event is subtracted using the FASTJET algorithm [49, 51, 85].

\subsubsection{Event Selection and Categorization}

Selections based isolation and identification requirements are used to reduce background in which the physical process does not produce four leptons, e.g. $Z+j e t$ events, generally referred to as reducible backgrounds. All reconstructed leptons are also required to have an impact parameter which is sufficiently compatible with the primary vertex [86].

Events are then classified into a number of categories. Categories which make up the signal region always consist of events with two oppositely charged lepton pairs. 


\section{CHAPTER 4. HIGGS SEARCHES WITH ZZ DECAYS}

The signal regions are then further subdivided into categories based on the number of jets which provides sensitivity to various production mechanisms, especially VBF where at least two additional jets are always produced. Events are either in the dijet tag category if there are at least two jets or in the non-dijet category if there are less than two jets. Events are also classified according to final state lepton flavors (4e, $4 \mu, 2 e 2 \mu)$. Since each flavor will have a different $m_{4 \ell}$ resolution, this categorization increases the overall sensitivity to signal events. Control regions in which either looser ID requirements or same-sign leptons pairs are used. These control regions are used to estimate the amount of instrumental background from data.

Minimal kinematic selections are applied to further reduce the continuum ZZ backgrounds. In order to reduce the contamination of low-mass resonances, such as $J / \psi$ 's, all dilepton pairings are required to have a minimum invariant mass, $m_{\ell \ell}>$ $4 \mathrm{GeV}$. Dilepton pairings whose invariant mass is closest to the $\mathrm{Z}$ pole-mass is referred to as $Z_{1}$, while the other pairing is referred to as $Z_{2}$. The invariant mass of these dilepton pairs is denoted by $m_{1}$ and $m_{2}$, respectively, and are required to satisfy $12<m_{2}<120 \mathrm{GeV}$ and $40<m_{1}<120 \mathrm{GeV}$. The leading and subleading leptons are required to have $p_{T}>20$ and $p_{T}>10 \mathrm{GeV}$, respectively.

\subsubsection{Yields and Kinematics Distributions}

The expected shape and event yields for continuum ZZ backgrounds are taken from MC simulation. Cross sections for $q \bar{q}$ annihilation and gg initiated events are 


\section{CHAPTER 4. HIGGS SEARCHES WITH ZZ DECAYS}

calculated at NLO using MCFM. Systematic variations due to QCD renormalization scale, factorization scale, and parton distribution functions are calculated as a function of $m_{4 \ell}$ following the PDF4LHC prescription $[87,88]$. The total uncertainties from QCD and PDFs are typically $8 \%$.

Expected event yields for the reducible background is estimated by deriving an extrapolation between loose and tight identification requirements. Event in the signal region are then extrapolated from a separate control region [86].

Systematic uncertainties are evaluated from data for trigger and combined lepton reconstruction, identification, and isolation efficiencies using the tag \& probe method. Samples of $Z \rightarrow \ell \ell, \Upsilon \rightarrow \ell \ell$, and $J / \psi \rightarrow \ell \ell$ events are used to set and validate the absolute momentum scale and momentum resolution. Additional systematics arise from limited statistics in background control regions as well as systematic differences between the control regions.

Starting from Higgs boson production cross sections described in Section 4.2.1, signal event yields are calculated using MC simulations to calculate efficiencies. Shapes of signal distributions are also taken from MC simulations.

There are a number of different measurables with which event likelihoods will be

evaluated. For cross section measurements, $m_{4 \ell}, \mathscr{D}_{\mathrm{bkg}}^{\mathrm{kin}}$, and either $\mathscr{D}_{j}$ et or $p_{T, 4 \ell}$ are used. The first two variables provide discrimination between signal and background, while the latter two distinguish different production modes. $\mathscr{D}_{\mathrm{bkg}}^{\mathrm{kin}}$ is a discriminant built within the MELA framework presented in Chapter 3 and is described in Equa- 


\section{CHAPTER 4. HIGGS SEARCHES WITH ZZ DECAYS}

tion 3.10. $\mathscr{D}_{\text {jet }}$ is used for events in the dijet category and is a linear combination of the difference in pseudorapidity, $\Delta \eta$, and the invariant mass of the event's two leading jets, $m_{j j}$. The coefficients which are used in $\mathscr{D}_{\text {jet }}$ were optimized for maximal separation between VBF events and gluon-gluon fusion events. For events in the non-dijet category, $p_{T, 4 \ell}$ is used to distinguish different production mechanisms.

The signal and background $m_{4 \ell}$ distributions are described using empirical functions, $\mathscr{P}_{b k g}\left(m_{4 \ell}\right)$ and $\mathscr{P}_{\text {sig }}\left(m_{4 \ell} ; m_{H}\right)$. The signal modeling is derived by interpolating function parameter from fit to individual Higgs mass hypotheses to intermediate masses, similar to the semi-leptonic analysis. To account for the correlation between $m_{4 \ell}$ and other variables, conditional probability distributions are built, $\mathscr{P}\left(\mathscr{D}_{\mathrm{bkg}}^{\mathrm{kin}} \mid m_{4 \ell}\right)$, $\mathscr{P}\left(\mathscr{D}_{\text {jet }} \mid m_{4 \ell}\right)$, and $\mathscr{P}\left(p_{T, 4 \ell} \mid m_{4 \ell}\right)$. In this way, three separate likelihoods can be constructed to describe each event class: using a single measurable, $m_{4 \ell}$; a 2D likelihood described by

$$
\mathscr{L}_{2 D} \sim \mathscr{P}_{b k g}\left(m_{4 \ell}\right) \mathscr{P}_{b k g}\left(\mathscr{D}_{b k g}^{k i n} \mid m_{4 \ell}\right)+\mu \times \mathscr{P}_{\text {sig }}\left(m_{4 \ell} ; m_{H}\right) \mathscr{P}_{s i g}\left(\mathscr{D}_{b k g}^{k i n} \mid m_{4 \ell}\right) ;
$$

or using all three measurables according to

$$
\begin{array}{r}
\mathscr{L}_{3 D}\left(m_{4 \ell}, \mathscr{D}_{b k g}^{k i n}, \mathscr{D}_{j e t}\right) \sim \mathscr{P}_{b k g}\left(m_{4 \ell}\right) \mathscr{P}_{b k g}\left(\mathscr{D}_{b k g}^{k i n} \mid m_{4 \ell}\right) \mathscr{P}_{b k g}\left(\mathscr{D}_{V B F} \mid m_{4 \ell}\right)+ \\
\mu \times \mathscr{P}_{\text {sig }}\left(m_{4 \ell} ; m_{H}\right) \mathscr{P}_{s i g}\left(\mathscr{D}_{b k g}^{k i n} \mid m_{4 \ell}\right) \mathscr{P}_{s i g}\left(\mathscr{D}_{V B F} \mid m_{4 \ell}\right),
\end{array}
$$

where $\mathscr{D}_{V B F}$ is used as short hand for either $p_{T, 4 \ell}$ or $\mathscr{D}_{\text {jet }}$, depending on which category 


\section{CHAPTER 4. HIGGS SEARCHES WITH ZZ DECAYS}

the event belongs to.

For certain property measurements, distributions of $\mathscr{D}_{J^{P}}$ and $\mathscr{D}_{\mathrm{bkg}}$ are used. The $\mathscr{D}_{\mathrm{bkg}}$ variable is an extension of $\mathscr{D}_{\mathrm{bkg}}^{\mathrm{kin}}$ which also includes $m_{4 \ell}$ information for optimal separation of signal and background,

$$
\mathscr{D}_{b k g}=\left(1+\frac{\mathscr{P}_{b k g}^{k i n}\left(m_{Z_{1}}, m_{Z_{2}}, \vec{\Omega} \mid m_{4 \ell}\right) \times \mathscr{P}_{b k g}^{\text {mass }}\left(m_{4 \ell}\right)}{\mathscr{P}_{0^{+}}^{\text {kin }}\left(m_{Z_{1}}, m_{Z_{2}}, \vec{\Omega} \mid m_{4 \ell}\right) \times \mathscr{P}_{\text {sig }}^{\text {mass }}\left(m_{4 \ell}\right)}\right)^{-1} .
$$

Although spin-0 models are inherently production independent, spin-1 and spin-2 models can have information of the production mechanism reflected in distributions of the production angles through spin correlations. In order to be more model independent when testing alternative signal models, discriminants can be designed such that production angles are integrated out making the discriminant independent of the production mechanism. A third set of variables which are production independent, $D_{b k g}^{d e c}$ and $D_{J^{P}}^{d e c}$, will also be used to test spin-1, and spin-2 models. In these cases, the likelihood used for spin-parity measurements is constructed from two observables, $\mathscr{L}\left(\mathscr{D}_{\mathrm{bkg}}, \mathscr{D}_{J^{P}}\right)$, or their production independent forms.

The input matrix element calculations used for signal events are the analytical descriptions discussed in Section 3 and the JHUGen squared matrix element. These calculations were checked against each other and were found to perform the same in the $2 e 2 \mu$ channel. JHUGen is used since it has more processes implemented. Background matrix element calculations are taken from MCFM. 


\section{CHAPTER 4. HIGGS SEARCHES WITH ZZ DECAYS}

The expected distributions that are used to build likelihoods are taken from MC

simulation for both the signal and continuum backgrounds. The $\mathscr{D}_{\mathrm{bkg}}^{\mathrm{kin}}$ and $\mathscr{D}_{J^{P}}$ distributions for the reducible background control regions are found to be similar to those of the continuum ZZ backgrounds. Because of the lack of statistics in the control regions, the continuum background distributions are used and then corrected to match the average shape in the opposite sign control regions. The difference between the control region shapes and the continuum ZZ shapes are taken as a systematic uncertainty on the reducible background.

\subsubsection{Observation}

The expected and observed event yields for the different event classes is shown in tables 4.6, 4.7. The expected and observed $m_{4 \ell}$ distribution is show in Figure 4.11. The expected and observed distribution of events in the $m_{4 \ell}-K_{D}$ plane are shown in Figure 4.12. Finally, expected and observed distributions of events in the $m_{4 \ell}-p_{T, 4 \ell}$ and $m_{4 \ell}-\mathscr{D}_{\text {jet }}$ plane are shown in Figure 4.13. The data show a clear excess of events around $m_{4 \ell}=126 \mathrm{GeV}$. Elsewhere, no significant deviations from the background only expectation are found. Events near the signal peak also tend to be distributed closer to $\mathscr{D}_{\mathrm{bkg}}^{\mathrm{kin}}=1$, consistent with that of a Higgs-like signal, as demonstrated in Figure 4.12.

To quantify the statistical significance of the observed data with respect to signal and background expectation, fits are done using either the 1D, 2D, or 3D likeli- 

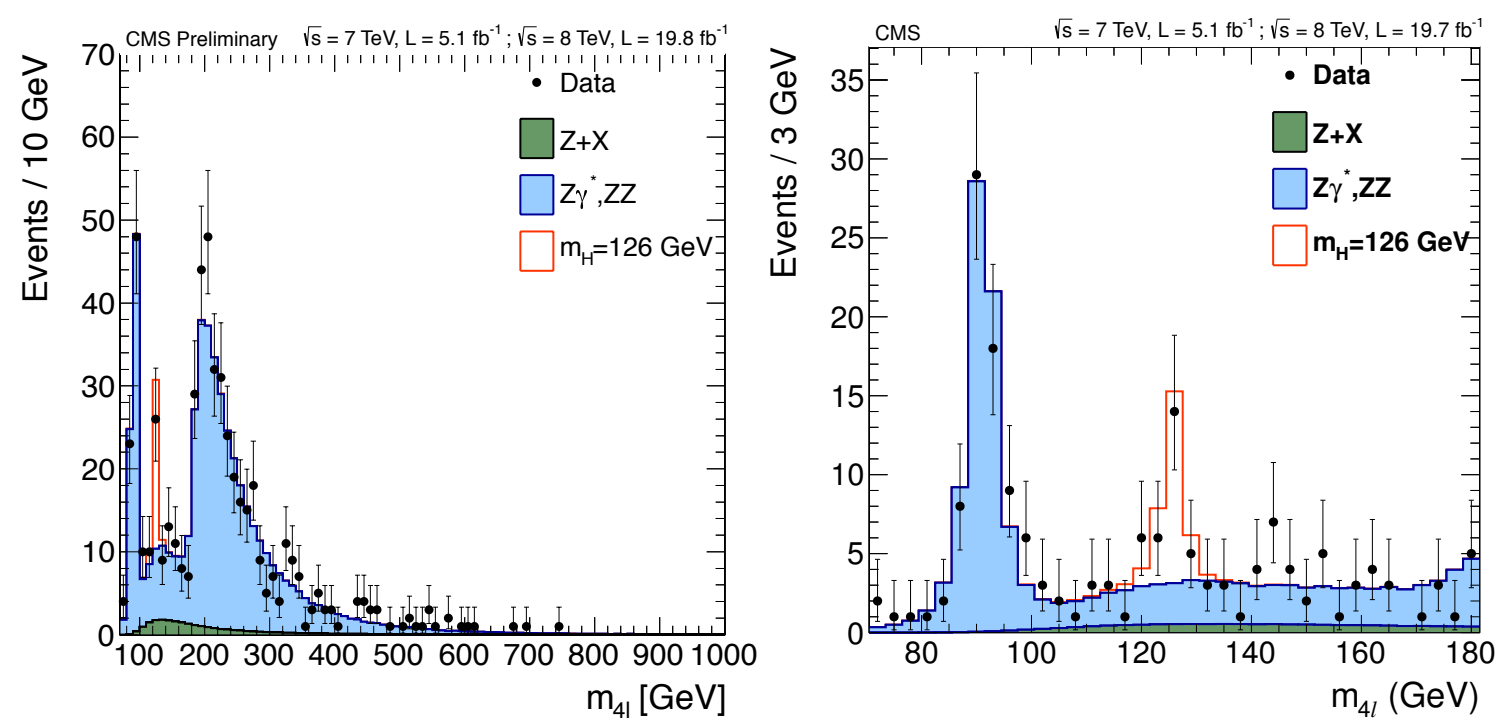

Figure 4.11: Invariant mass distribution of the $4 \ell$ system for events between $70<$ $m_{4 \ell}<1000 \mathrm{GeV}$ (left) and between $100<m_{4 \ell}<180 \mathrm{GeV}$ (right). All final states have been included. Points with error bars represent a sum of the $\sqrt{s}=7 \mathrm{TeV}$ and $\sqrt{s}=8 \mathrm{TeV}$ datasets. Solid histograms represent background estimations. The open red histogram represents simulation of a SM Higgs, $m_{H}=126 \mathrm{GeV}$.
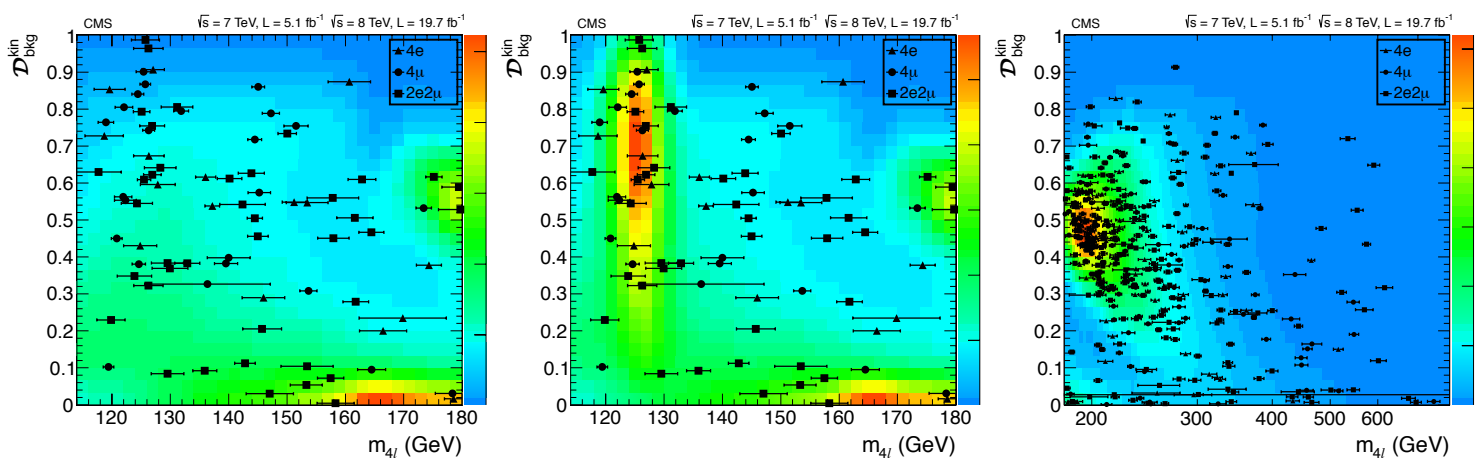

Figure 4.12: Distribution of $m_{4 \ell}$ and $K_{D}$ in various regions. Contours in the left and right plot represent the background expectation of continuum ZZ events. Contours in the middle plot represent signal plus background expectation, where signal is a SM Higgs, $m_{H}=126 \mathrm{GeV}$. Points with error bars represent the individual events observed in the four different final states. Horizontal error bars represent the reconstructed mass uncertainties. 

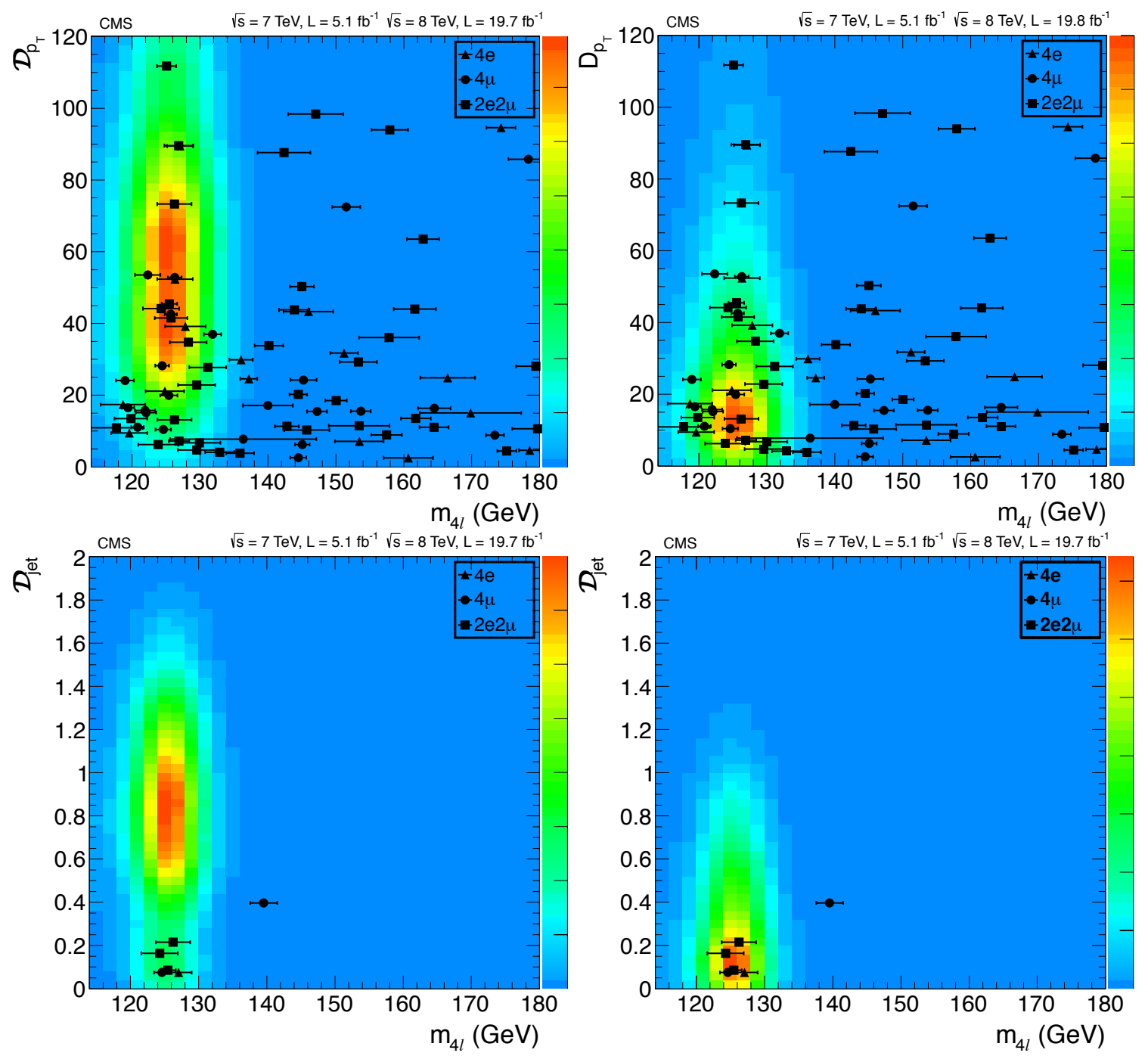

Figure 4.13: Distribution of $p_{T, 4 \ell}$ in the non-dijet category (top row) and $\mathscr{D}_{\text {jet }}$ in the dijet category (bottom row) for expectation of a VBF produced (left column) or a gluon-gluon fusion produced Higgs boson with $m_{H}=126 \mathrm{GeV}$. Points with error bar show the distribution of observed $4 \mu$ (circles), $4 e$ (triangles), and $2 e 2 \mu$ (squares) events. 


\section{CHAPTER 4. HIGGS SEARCHES WITH ZZ DECAYS}

hood as described in the previous section. Compatibility of data with respect to the background only hypothesis can also be quantified in terms of $95 \%$ confidence level upper limits of $\mu$. Aside from the significant deviation from expectation near $126 \mathrm{GeV}$, the observed upper limits are always consistent with expectation to within $2 \sigma$. The current data is sufficient to rule out SM Higgs mass hypotheses between $129.5<m_{H}<832 \mathrm{GeV}$ and between $114.5<m_{H}<119$ at $95 \%$ confidence level. The large deviation from the expected limit around $126 \mathrm{GeV}$ is a reflection of the excess of events in this region. The p-value scan as a function of the hypothetical Higgs mass is shown in Figure 4.15. The minimum local p-value occurs around 125.7 GeV and has a value of $6.8 \sigma$. This significant deviation from the backgroundonly hypothesis has a cross section which is compatible with that expected from the SM Higgs. The ratio of the best-fit cross section with respected to the expected

SM Higgs cross section if found to be $\mu=\sigma_{o b s} / \sigma_{S M}=0.93_{-0.24}^{+0.29}$. Figure 4.16 shows the best-fit value in both the dijet $\left(\mu=1.45_{-0.62}^{+0.89}\right)$ and the untagged $\left(\mu=0.83_{-0.25}^{+0.31}\right)$ categories, as well as the combined.

\begin{tabular}{lcccc}
\hline Channel & $4 e$ & $4 m u$ & $2 e 2 \mu$ & $4 \ell$ \\
\hline$Z Z$ background & $1.1 \pm 0.1$ & $2.5 \pm 0.2$ & $3.2 \pm 0.2$ & $6.8 \pm 0.3$ \\
$Z+$ X background & $0.8 \pm 0.2$ & $0.4 \pm 0.2$ & $1.3 \pm 0.3$ & $2.6 \pm 0.4$ \\
\hline All backgrounds & $1.9 \pm 0.2$ & $2.9 \pm 0.2$ & $4.6 \pm 0.4$ & $9.4 \pm 0.5$ \\
\hline$m_{H}=125 \mathrm{GeV}$ & $3.0 \pm 0.4$ & $6.4 \pm 0.7$ & $7.9 \pm 1.0$ & $17.3 \pm 1.3$ \\
$m_{H}=126 \mathrm{GeV}$ & $3.4 \pm 0.5$ & $7.2 \pm 0.8$ & $9.0 \pm 1.1$ & $19.6 \pm 1.5$ \\
\hline Observed & 4 & 8 & 13 & 25 \\
\hline
\end{tabular}

Table 4.6: Expected and observed yields in the mass range $121.5<m_{4 \ell}<130.5$ for different event classes. 


\section{CHAPTER 4. HIGGS SEARCHES WITH ZZ DECAYS}

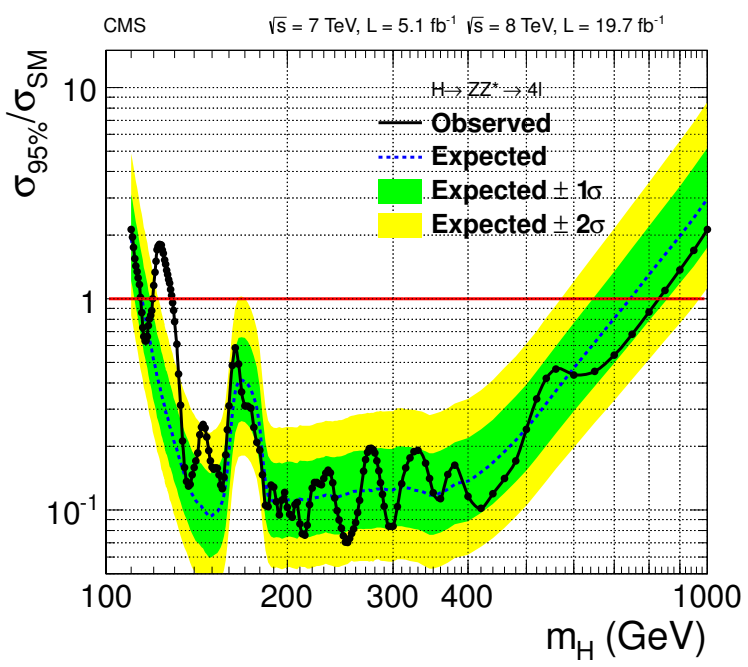

Figure 4.14: Expected and observed 95\% confidence level upper limit on $\sigma / \sigma_{S M}$ as a function of the hypothetical Higgs mass, $m_{H}$, in the range [110-1000]. The green and yellow bands represent the one and two sigma bands of the expected distribution, respectively.
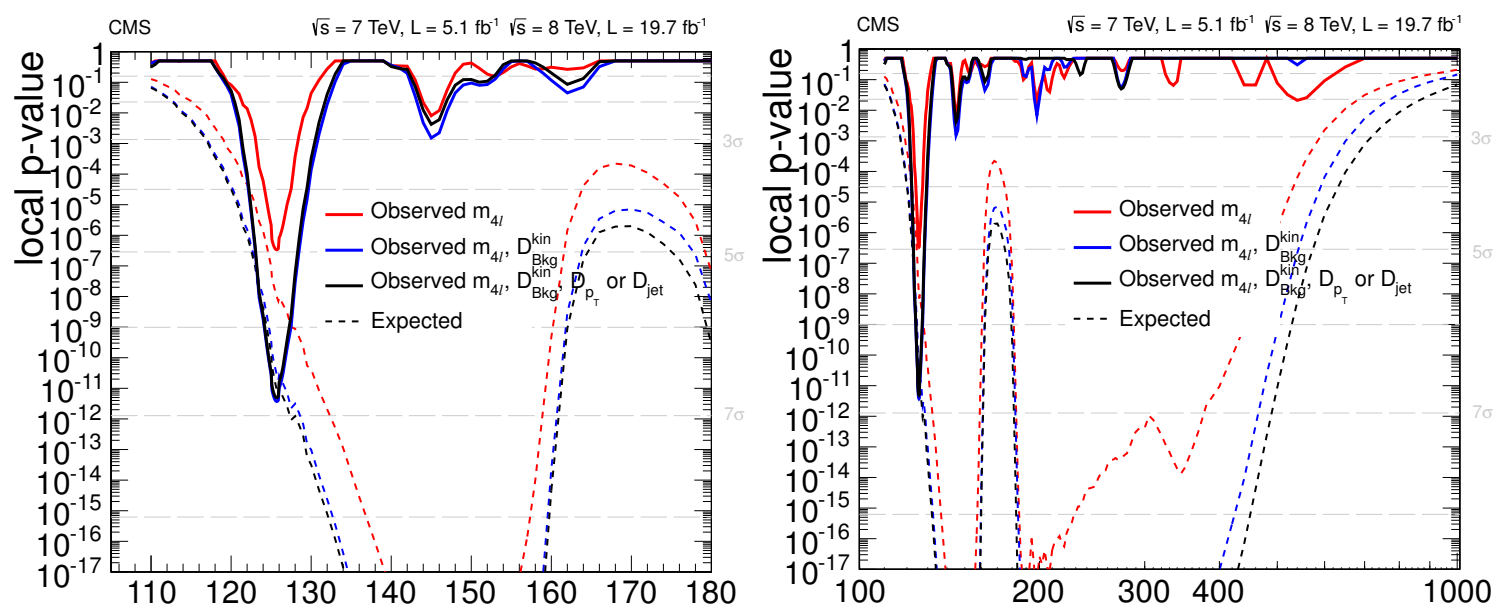

Figure 4.15: Expected and observed p-value with respect to the background only hypothesis as a function of the hypothetical Higgs mass, $m_{H}$, in the range [110-180] (left) and [110-1000] (right). Solid lines show the observed p-values while dashed lines show the expected p-values, assuming a SM Higgs. Green lines show p-values obtained using only the information about $m_{4 \ell}$ distributions. Red lines show p-values obtained using $m_{4 \ell}$ vs $K_{D}$ distributions. 


\section{CHAPTER 4. HIGGS SEARCHES WITH ZZ DECAYS}

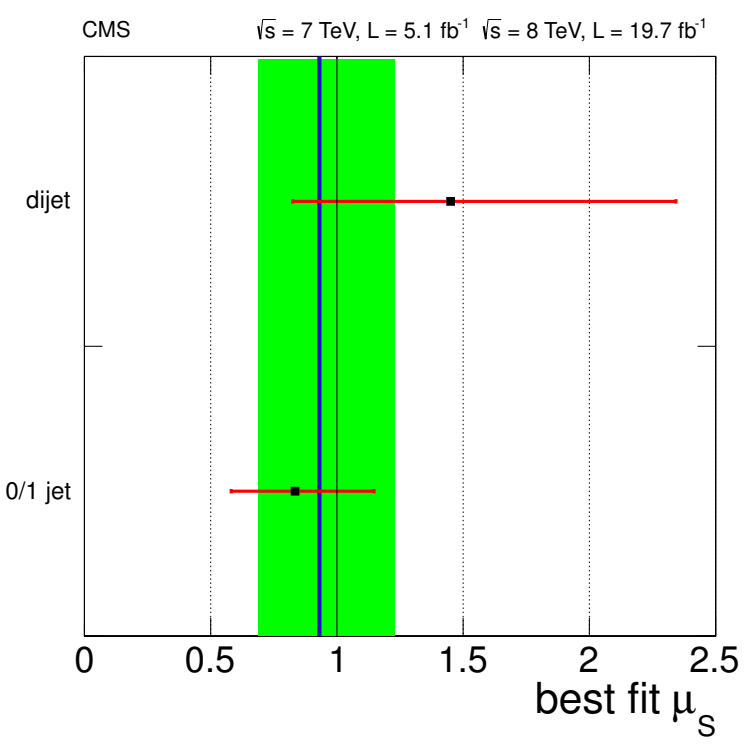

Figure 4.16: Best fit signal strength modifier, $\mu$, is both the dijet and untagged categories as well the combination of all channels (black line). Red bar represent the $68 \%$ confidence intervals for each of the individual measurements. The green band represents the $68 \%$ confidence interval for the combined measurement.

\subsubsection{Spin and Parity Measurements}

Assuming two basic conservation laws, electric charge and angular momentum, one can infer that the excess of events presented above corresponds to a new chargeless, bosonic resonance. However, little else can be concluded from the above data alone

\begin{tabular}{lcccc}
\hline Channel & $4 e$ & $4 \mu$ & $2 e 2 \mu$ & $4 \ell$ \\
\hline$Z Z$ background & $77.1 \pm 10.4$ & $119.4 \pm 15.1$ & $190.6 \pm 24.5$ & $387.1 \pm 30.6$ \\
$Z+$ X background & $7.4 \pm 1.5$ & $3.6 \pm 1.5$ & $11.5 \pm 2.9$ & $22.6 \pm 3.6$ \\
\hline All backgrounds & $84.6 \pm 10.5$ & $123.51 \pm 15.2$ & $202.1 \pm 24.6$ & $409.7 \pm 30.8$ \\
\hline$m_{H}=500 \mathrm{GeV}$ & $5.2 \pm 0.6$ & $7.1 \pm 0.8$ & $12.2 \pm 1.4$ & $24.5 \pm 1.7$ \\
$m_{H}=800 \mathrm{GeV}$ & $0.7 \pm 0.1$ & $0.9 \pm 0.1$ & $1.6 \pm 0.2$ & $3.1 \pm 0.2$ \\
\hline Observed & 89 & 134 & 247 & 470 \\
\hline
\end{tabular}

Table 4.7: Expected and observed yields in the mass range $100<m_{4 \ell}<1000$ for difference class of events. 


\section{CHAPTER 4. HIGGS SEARCHES WITH ZZ DECAYS}

since beyond the SM resonances could mimic the above signatures. Understanding whether or not this new boson is the SM Higgs, one of several Higgses, or even something more exotic, like a graviton, is one of the most promising routes to searching for physics beyond the SM. As demonstrated in Chapter 3, the MELA techniques can be employed to perform property measurements and infer more information about the observed resonance.

Hypothesis testing can be used to evaluate the compatibility of data with respect to either the null hypothesis, the SM background plus a SM Higgs boson, or some alternative signal hypothesis. The list of alternative signal hypotheses include: $J^{P}=$ $0^{-}, 0_{h}^{+}, q \bar{q} \rightarrow 1^{-}, q \bar{q} \rightarrow 1^{+}, g g \rightarrow 2_{m}^{+}, g g \rightarrow 2_{h}^{+}, g g \rightarrow 2_{h}-, q \bar{q} \rightarrow 2_{m}^{+}$, and $g g \rightarrow 2_{b}^{+}$ and are described in Chapter 3 . In each case, a dedicated discriminant is built, $\mathscr{D}_{J^{P}}$, and used to distinguish kinematics of a SM Higgs boson from the alternative signal hypothesis.

The expected and observed $\mathscr{D}_{b k g}$ and $\mathscr{D}_{b k g}^{d e c}$ distributions are shown in Figure 4.17. Although the $\mathscr{D}_{b k g}$ distributions of some alternative signals are more background like compared to the SM Higgs, these variations are typically small compared to the difference between each signal and background. Thus, this variable serves as a sufficient, model independent way of isolating signal events. The distribution of each of the $D_{J^{P}}$ variables is shown in Figure 4.18 for events which satisfy $D_{b k g}>0.5$. Each plot shows that the SM Higgs tends to be distributed more towards $D_{J^{P}}=1$ while the corresponding alternative signal is distributed more towards $D_{J^{P}}=0$. 


\section{CHAPTER 4. HIGGS SEARCHES WITH ZZ DECAYS}
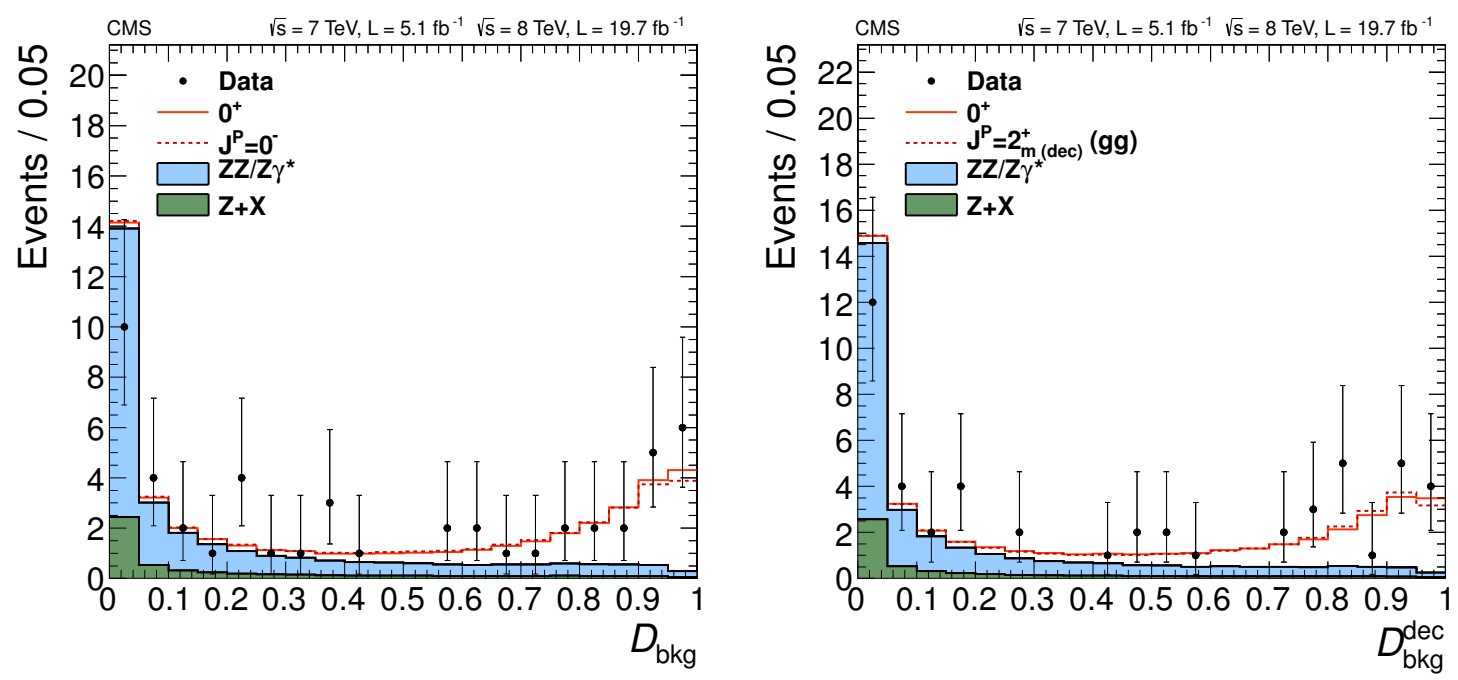

Figure 4.17: Distributions of $\mathscr{D}_{b k g}$ (left) and $\mathscr{D}_{b k g}$ (right). Expected distribution for a $125.6 \mathrm{GeV}$ SM Higgs boson is shown in red, the continuum ZZ background in blue, and the reducible background in green.

The effect of different couplings on the ZZ branching ratios as well as different relative efficiencies is accounted for by calculating correction factors for each of the six different channels comparing SM Higgs against alternative $J^{P}$ samples with JHUGen. Tables 4.8, 4.9, 4.10, and 4.11 show each of these correction factors for all alternative signals in all channels. The large difference in the $q \bar{q}$ initiated samples are due to the more forward rapidity distributions of these samples relative to the $g g$ initiated samples.

The test statistic used to distinguish the null hypothesis from the alternative hypothesis is a log-likelihood ratio, $q=-2 \ln \left(\mathscr{L}_{S M} / \mathscr{L}_{J^{P}}\right)$. Expected results are obtained in two different ways: generating pseudoexperiments using the SM Higgs cross section for each hypothesis or using the best-fit signal strength modifier, $\mu$, 


\section{CHAPTER 4. HIGGS SEARCHES WITH ZZ DECAYS}
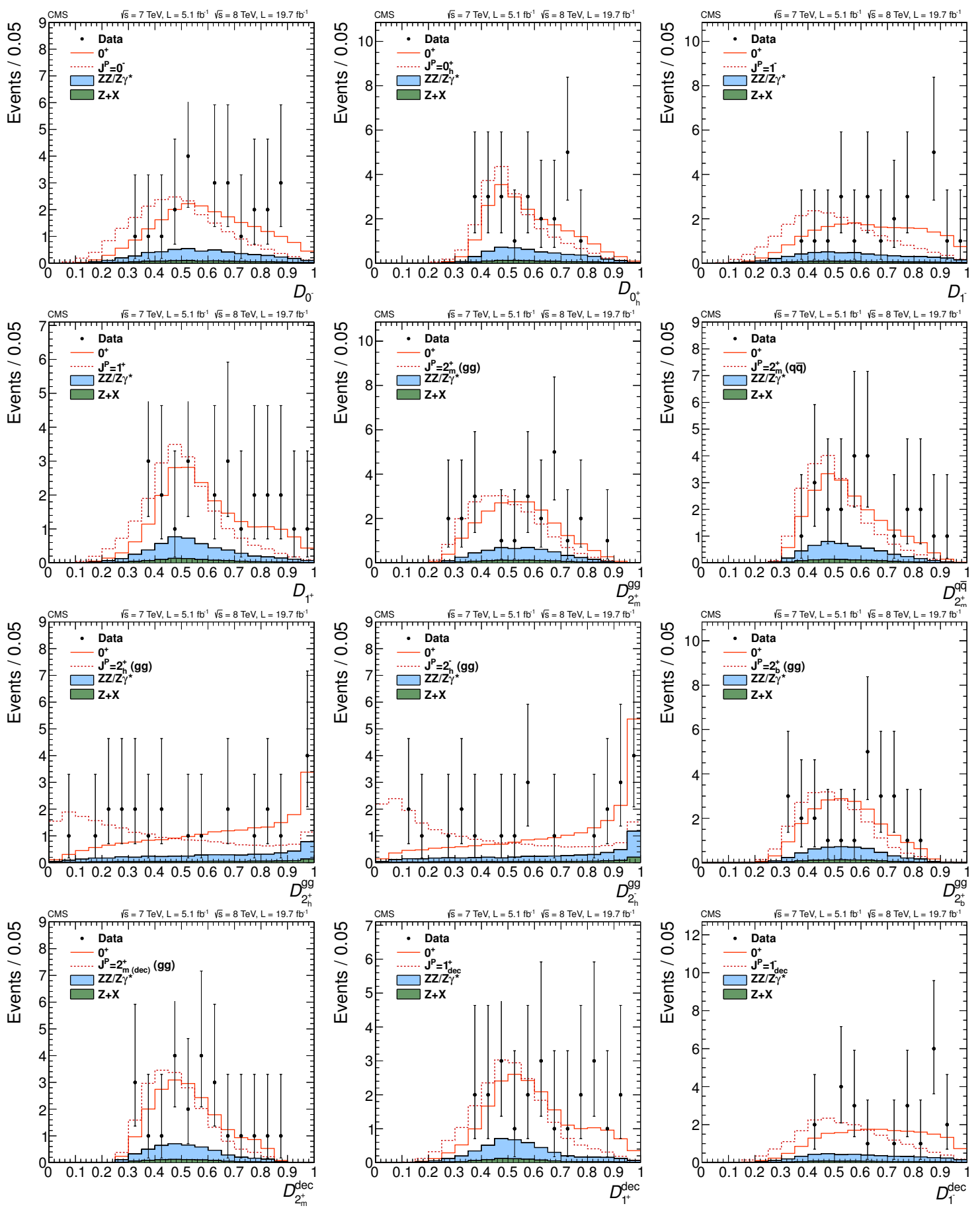

Figure 4.18: Distributions of $\mathscr{D}_{J^{P}}$ for $J^{P}=0^{-}, 0_{h}^{+}$, and $1^{-}$(first row), $J^{P}=1^{+}$, $2_{m}^{+}(g g)$, and $2_{m}^{+}(q \bar{q})$ (second row), $J^{P}=2_{h}^{+}, 2_{h}^{-}$, and $2_{b}^{+}$(third row), and production independent tests of $J^{P}=1^{-}, 1^{+}$, and $2_{m}^{+}$(fourth row). Expected shapes for a 125.6 GeV SM Higgs boson is shown in red, the continuum background in blue, the reducible background in green, and observed data in the point with error bars. 


\section{CHAPTER 4. HIGGS SEARCHES WITH ZZ DECAYS}

for each hypothesis individually. Since the expected production cross section for alternative signal models is highly model dependent, using the best-fit signal strength for generating toys allows for a more model independent interpretation.

Results are shown in Table 4.12 where observed $0^{+}\left(J^{P}\right)$ refers to the p-value of the observed test statistic, represented by the red arrow in Figure 4.19, calculated according to the SM (alternative signal) toy distribution, shown in yellow (blue), converted to normal quantiles. A $C L_{s}$ criterion is built from the p-values according to:

$$
C L_{s}=P\left(q>q_{0} \mid S M\right) / P\left(q>q_{0} \mid J^{P}\right)
$$

All results show that data is more consistent with the Higgs boson expectation and disfavor the alternative hypothesis at a level of $8.1 \%$ or better.

Several results show large observed significance with respect to the expected, namely the $1^{+}, 1^{-}$, and $2_{m, q \bar{q}}^{+}$tests. Each of these cases have $m_{1}$ and $m_{2}$ distributions which are quite distinct from a SM Higgs boson. As a result of a statistical fluctuation observed in data in the tails of these distributions, these models all have large q-values. This is one of the driving factors to why the discovery significance is larger for the 2D analysis. However, it is important to note that these results are correlated due to this statistical fluctuation. 


\section{CHAPTER 4. HIGGS SEARCHES WITH ZZ DECAYS}
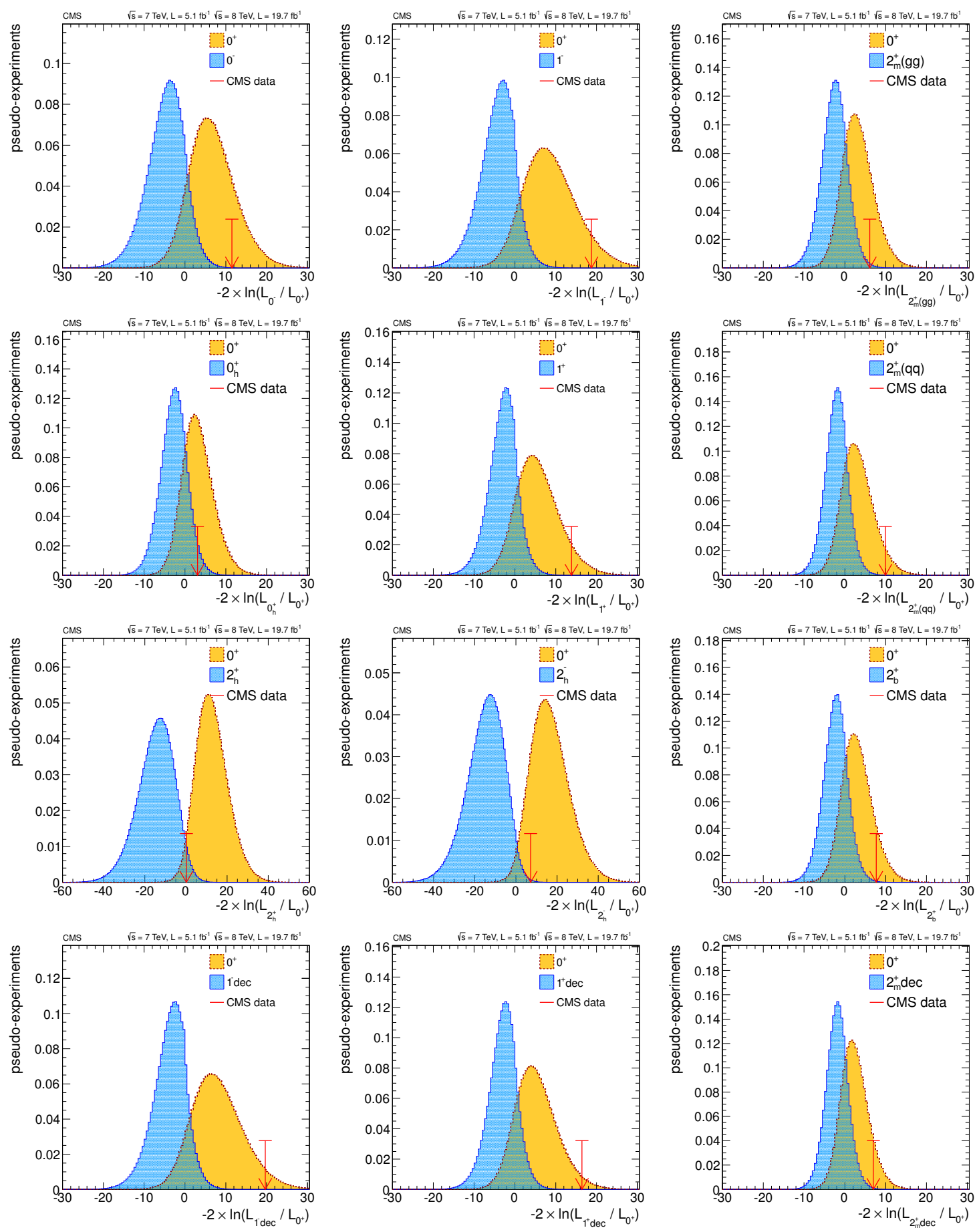

Figure 4.19: Distribution of expected and observed test statistics for various hypothesis test. Orange histograms represent toys generated under the null hypothesis, SM background plus a SM Higgs boson. Blue histograms represent toys generated under the alternative hypothesis. The red arrow shows the value of the observed test statistic. All resonances are assumed to have a mass of $125.6 \mathrm{GeV}$. 


\section{CHAPTER 4. HIGGS SEARCHES WITH ZZ DECAYS}

\subsubsection{Constraining CP-violation}

As discussed in Chapter 1, SUSY and other 2HDMs can produce a parity-violating interactions. Thus, constraining CP-violation in the HZZ amplitude is one of the most promising ways of probing new physics beyond the SM which could help to explain not only theoretical problems the SM is thought to suffer from, e.g. fine tuning, but empirical facts the SM is currently thought to be insufficient to explain.

The parameter $f_{a 3}{ }^{3}$ is a natural gauge of CP-violation in the HZZ amplitude. Given that $f_{a 3}=1$ has been ruled out through hypothesis testing in favor of the SM Higgs hypothesis at the level of $3.6 \sigma$, measuring any non-zero value of $f_{a 3}$ would be direct evidence of CP-violation, if $f_{a 2}=0$. Furthermore, the $D_{0^{-}}$variable used for hypothesis testing in Section 4.2.5 is suitable for measuring the value of $f_{a 3}$ using the simplified model for a mixed-CP state described in Equation 3.9 (see Chapter 3).

Using this model, a two parameter fit for $\mu$ and $f_{a 3}$ was performed. Figure 4.20 shows the $\ln \mathscr{L}$ scan as a function of the two parameters. Profiling $\mu$, we arrive at the 1D $\ln \mathscr{L}$ scan versus $f_{a 3}$ in Figure 4.21 . The expected $68 \%$ and $95 \%$ confidence level intervals, from fitting the Asimov dataset ${ }^{4}$, are found to be $[0.0,0.39]$ and $[0.0,0.74]$, respectively. The observed $68 \%$ and $95 \%$ confidence level intervals are found to be $[0.00,0.17]$ and $[0.00,0.51]$, respectively.

\footnotetext{
${ }^{3}$ The definition of $f_{a 3}$ is equivalent to $f_{g 4}$ defined in Chapter 3

${ }^{4}$ Asimov datasets provide representative datasets which can be used to approximate experimental sensitivity asymptotically. This procedure is motivated in reference [89].
} 


\section{CHAPTER 4. HIGGS SEARCHES WITH ZZ DECAYS}

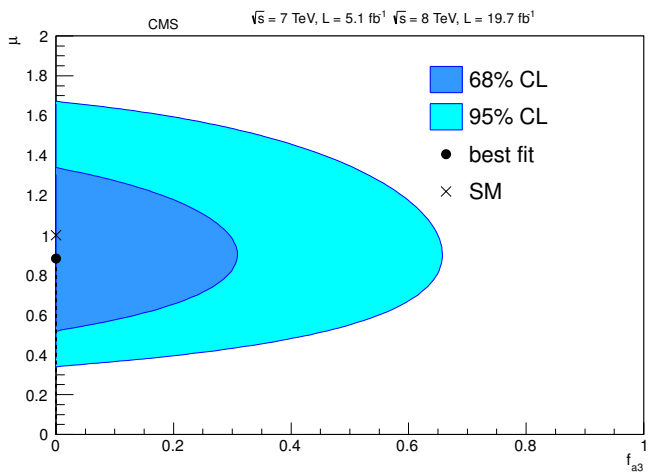

Figure 4.20: Distribution of $-2 \ln \mathscr{L}$ versus $\left(\mu, f_{a 3}\right)$, Blue and teal band represent the $68 \%$ and $95 \%$ confidence level contours, respectively. The point represents the location of the maximum likelihood.

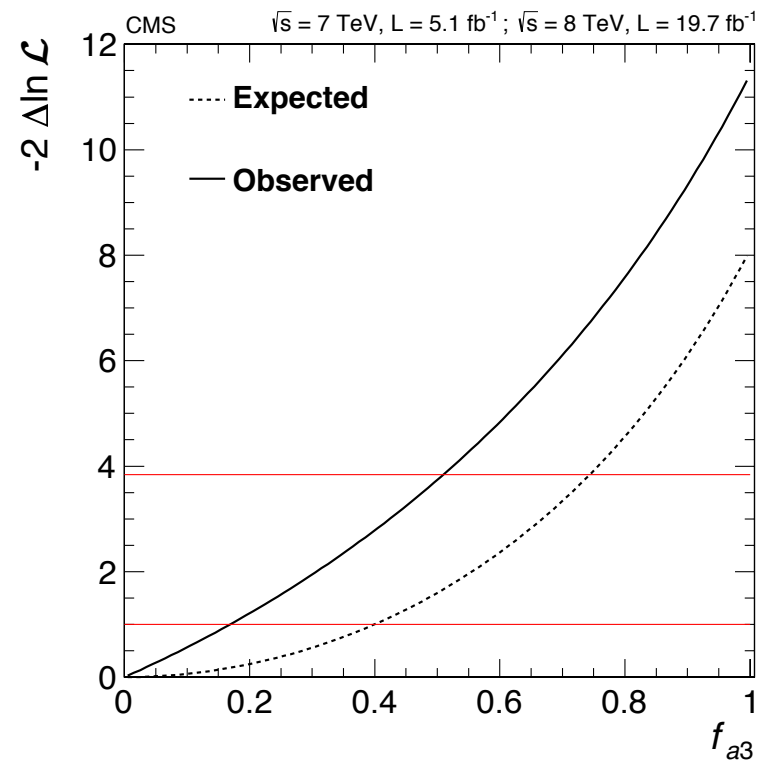

Figure 4.21: Distribution of $-2 \ln \mathscr{L}$ versus $f_{a 3}$. The black line in the right plots represents the expected distribution calculated from fitting the Asimov dataset; the blue line represents the observed distribution. The signal strength, $\mu$, has been profiled. 


\section{CHAPTER 4. HIGGS SEARCHES WITH ZZ DECAYS}

\subsection{Summary}

A search for a SM Higgs boson decaying into two Z boson which subsequently decay into to quark jets and two leptons has been presented. The data used in this analysis constitute $4.6 \mathrm{fb}^{-1}$ of integrated luminosity. No significant excess of events was found and upper limits on the observed cross section have been measured relative to the SM model expectation. Higgs boson masses in the range $[340,390]$ have been ruled out. More data should allow sensitivity which is sufficient for excluding almost the entire range between 200 and $600 \mathrm{GeV}$ with this channel alone.

A search for a SM Higgs boson decaying into two Z boson which subsequently decay into 4 lepton has been presented. The data used in this analysis constitute $5.1 f b^{-1}$ and $19.7 f b^{-1}$ at $\sqrt{s}=7$ and $8 \mathrm{TeV}$, respectively. An excess of events has been observed around $126 \mathrm{GeV}$. The properties of these events have been analyzed in the context of the mass and angular distributions of the final state product using the MELA techniques outlined in Chapter 3. Hypothesis testing shows that data is more consistent with the SM Higgs boson hypothesis with respect to all others tested, although results for the $2_{h}^{+}$are largely inconclusive. Measurement of the scalar model parameter $f_{a 3}$ has also been presented and found to be consistent with zero. The $95 \%$ confidence interval is $[0.0,0.51]$, thus providing a direct constraint on the level of CP-violation in the HZZ amplitude. At other values of $m_{4 \ell}$, the data is consistent with the background only hypothesis. In light of this, limits have been set on $\sigma / \sigma_{S M}$

and SM Higgs boson masses in the range [114.5,119] and $[129.5,832]$ have been ruled 
CHAPTER 4. HIGGS SEARCHES WITH ZZ DECAYS

out. 


\section{CHAPTER 4. HIGGS SEARCHES WITH ZZ DECAYS}

Table 4.8: Table with correction factors and event yields in the different channels of the alternative spin-0 hypotheses arising due to lepton interference and detector effects.

\begin{tabular}{|c|c|c|c|c|c|c|c|}
\hline \multicolumn{8}{|c|}{$0_{m}^{+} \sqrt{s}=7 \mathrm{Tev}$} \\
\hline channel & $f_{i}^{J^{P}}$ & $\alpha_{\text {ideal }}(i)$ & $\epsilon_{\mathrm{reco}}(i)$ & $\alpha_{\exp }(i)$ & $N_{\exp }^{J^{P}}(i)$ & $\alpha_{\text {norm }}(i)$ & $N_{\text {norm }}^{J^{P}}(i)$ \\
\hline $4 \mathrm{e}$ & 0.2592 & 1.0 & 0.254878 & 1.0 & 0.681158 & 1.0 & 0.681158 \\
\hline $4 \mathrm{mu}$ & 0.2592 & 1.0 & 0.390734 & 1.0 & 1.05786 & 1.0 & 1.05786 \\
\hline $2 \mathrm{mu} 2 \mathrm{e}$ & 0.4816 & 1.0 & 0.305464 & 1.0 & 1.5215 & 1.0 & 1.5215 \\
\hline \multicolumn{8}{|c|}{$0_{m}^{+} \sqrt{s}=8 \mathrm{Tev}$} \\
\hline $4 \mathrm{e}$ & 0.2592 & 1.0 & 0.209051 & 1.0 & 2.83281 & 1.0 & 2.83281 \\
\hline $4 \mathrm{mu}$ & 0.2592 & 1.0 & 0.384041 & 1.0 & 5.20253 & 1.0 & 5.20253 \\
\hline $2 \mathrm{mu} 2 \mathrm{e}$ & 0.4816 & 1.0 & 0.279299 & 1.0 & 7.02377 & 1.0 & 7.02377 \\
\hline \multicolumn{8}{|c|}{$0^{-} \sqrt{s}=7 \mathrm{Tev}$} \\
\hline channel & $f_{i}^{J^{P}}$ & $\alpha_{\text {ideal }}(i)$ & $\epsilon_{\text {reco }}(i)$ & $\alpha_{\exp }(i)$ & $N_{\exp }^{J^{P}}(i)$ & $\alpha_{\text {norm }}(i)$ & $N_{\text {norm }}^{J^{P}}(i)$ \\
\hline $4 \mathrm{e}$ & 0.2382 & 0.845266 & 0.21946 & 0.730505 & 0.497589 & 0.847481 & 0.577268 \\
\hline $4 \mathrm{mu}$ & 0.2382 & 0.845266 & 0.375617 & 0.811788 & 0.858759 & 0.94178 & 0.996272 \\
\hline $2 \mathrm{mu} 2 \mathrm{e}$ & 0.5236 & 1.0 & 0.298035 & 0.974732 & 1.48305 & 1.13082 & 1.72054 \\
\hline \multicolumn{8}{|c|}{$0^{-} \sqrt{s}=8 \mathrm{Tev}$} \\
\hline $4 \mathrm{e}$ & 0.2382 & 0.845266 & 0.182517 & 0.736911 & 2.08753 & 0.854913 & 2.4218 \\
\hline $4 \mathrm{mu}$ & 0.2382 & 0.845266 & 0.358533 & 0.788697 & 4.10322 & 0.914991 & 4.76026 \\
\hline $2 \mathrm{mu} 2 \mathrm{e}$ & 0.5236 & 1.0 & 0.268579 & 0.962568 & 6.76086 & 1.1167 & 7.84348 \\
\hline \multicolumn{8}{|c|}{$0_{h}^{+} \sqrt{s}=7 \mathrm{Tev}$} \\
\hline channel & $f_{i}^{J^{P}}$ & $\alpha_{\text {ideal }}(i)$ & $\epsilon_{\text {reco }}(i)$ & $\alpha_{\exp }(i)$ & $N_{\exp }^{J^{P}}(i)$ & $\alpha_{\text {norm }}(i)$ & $N_{\text {norm }}^{J^{P}}(i)$ \\
\hline $4 \mathrm{e}$ & 0.2458 & 0.898313 & 0.271464 & 0.958688 & 0.653018 & 0.934054 & 0.636238 \\
\hline $4 \mathrm{mu}$ & 0.2458 & 0.898313 & 0.42079 & 0.951022 & 1.00605 & 0.926585 & 0.980197 \\
\hline $2 \mathrm{mu} 2 \mathrm{e}$ & 0.5084 & 1.0 & 0.340119 & 1.12178 & 1.70679 & 1.09296 & 1.66294 \\
\hline \multicolumn{8}{|c|}{$0_{h}^{+} \sqrt{s}=8 \mathrm{Tev}$} \\
\hline $4 \mathrm{e}$ & 0.2458 & 0.898313 & 0.223834 & 0.970414 & 2.749 & 0.945478 & 2.67836 \\
\hline $4 \mathrm{mu}$ & 0.2458 & 0.898313 & 0.412882 & 0.963257 & 5.01137 & 0.938505 & 4.8826 \\
\hline $2 \mathrm{mu} 2 \mathrm{e}$ & 0.5084 & 1.0 & 0.306175 & 1.09294 & 7.67655 & 1.06486 & 7.4793 \\
\hline
\end{tabular}




\section{CHAPTER 4. HIGGS SEARCHES WITH ZZ DECAYS}

Table 4.9: Table with correction factors and event yields in the different channels of the alternative spin-1 hypotheses arising due to lepton interference and detector effects.

\begin{tabular}{|c|c|c|c|c|c|c|c|}
\hline \multicolumn{8}{|c|}{$1^{-} \sqrt{s}=7 \mathrm{Tev}$} \\
\hline channel & $f_{i}^{J^{P}}$ & $\alpha_{\text {ideal }}(i)$ & $\epsilon_{\text {reco }}(i)$ & $\alpha_{\exp }(i)$ & $N_{\exp }^{J^{P}}(i)$ & $\alpha_{\text {norm }}(i)$ & $N_{\text {norm }}^{J^{P}}(i)$ \\
\hline $4 \mathrm{e}$ & 0.2395 & 0.854121 & 0.127888 & 0.429419 & 0.292502 & 0.89238 & 0.607852 \\
\hline $4 \mathrm{mu}$ & 0.2395 & 0.854121 & 0.207372 & 0.448064 & 0.47399 & 0.931127 & 0.985002 \\
\hline $2 \mathrm{mu} 2 \mathrm{e}$ & 0.521 & 1.0 & 0.167307 & 0.550292 & 0.837269 & 1.14357 & 1.73994 \\
\hline \multicolumn{8}{|c|}{$1^{-} \sqrt{s}=8 \mathrm{Tev}$} \\
\hline $4 \mathrm{e}$ & 0.2395 & 0.854121 & 0.100312 & 0.407292 & 1.15378 & 0.846397 & 2.39768 \\
\hline $4 \mathrm{mu}$ & 0.2395 & 0.854121 & 0.202707 & 0.451114 & 2.34693 & 0.937464 & 4.87718 \\
\hline $2 \mathrm{mu} 2 \mathrm{e}$ & 0.521 & 1.0 & 0.147179 & 0.528356 & 3.71105 & 1.09798 & 7.71197 \\
\hline \multicolumn{8}{|c|}{$1^{+} \sqrt{s}=7 \mathrm{Tev}$} \\
\hline channel & $f_{i}^{J^{P}}$ & $\alpha_{\text {ideal }}(i)$ & $\epsilon_{\text {reco }}(i)$ & $\alpha_{\exp }(i)$ & $N_{\exp }^{J^{P}}(i)$ & $\alpha_{\text {norm }}(i)$ & $N_{\text {norm }}^{J^{P}}(i)$ \\
\hline $4 \mathrm{e}$ & 0.2466 & 0.904082 & 0.151964 & 0.538705 & 0.366943 & 0.907252 & 0.617982 \\
\hline $4 \mathrm{mu}$ & 0.2466 & 0.904082 & 0.251755 & 0.57776 & 0.61119 & 0.973026 & 1.02933 \\
\hline $2 \mathrm{mu} 2 \mathrm{e}$ & 0.5068 & 1.0 & 0.198025 & 0.651177 & 0.990764 & 1.09667 & 1.66858 \\
\hline \multicolumn{8}{|c|}{$1^{+} \sqrt{s}=8 \mathrm{Tev}$} \\
\hline $4 \mathrm{e}$ & 0.2466 & 0.904082 & 0.119758 & 0.519051 & 1.47037 & 0.874151 & 2.4763 \\
\hline $4 \mathrm{mu}$ & 0.2466 & 0.904082 & 0.242716 & 0.572609 & 2.97901 & 0.964351 & 5.01706 \\
\hline $2 \mathrm{mu} 2 \mathrm{e}$ & 0.5068 & 1.0 & 0.177697 & 0.634913 & 4.45948 & 1.06928 & 7.51037 \\
\hline
\end{tabular}




\section{CHAPTER 4. HIGGS SEARCHES WITH ZZ DECAYS}

Table 4.10: Table with correction factors and event yields in the different channels of the alternative spin-2 hypotheses with minimal couplings arising due to lepton interference and detector effects.

\begin{tabular}{|c|c|c|c|c|c|c|c|}
\hline \multicolumn{8}{|c|}{$2_{m}^{+}(g g) \sqrt{s}=7 \mathrm{TeV}$} \\
\hline channel & $f_{i}^{J^{P}}$ & $\alpha_{\text {ideal }}(i)$ & $\epsilon_{\mathrm{reco}}(i)$ & $\alpha_{\exp }(i)$ & $N_{\exp }^{J^{P}}(i)$ & $\alpha_{\text {norm }}(i)$ & $N_{\text {norm }}^{J^{P}}(i)$ \\
\hline $4 \mathrm{e}$ & 0.2368 & 0.835494 & 0.22689 & 0.745966 & 0.508121 & 0.866069 & 0.58993 \\
\hline $4 \mathrm{mu}$ & 0.2368 & 0.835494 & 0.368471 & 0.785308 & 0.830746 & 0.911745 & 0.964499 \\
\hline $2 \mathrm{mu} 2 \mathrm{e}$ & 0.5265 & 1.0 & 0.296789 & 0.97203 & 1.47894 & 1.12853 & 1.71706 \\
\hline \multicolumn{8}{|c|}{$2_{m}^{+}(g g) \sqrt{s}=8 \mathrm{TeV}$} \\
\hline $4 \mathrm{e}$ & 0.2368 & 0.835494 & 0.18665 & 0.744846 & 2.11001 & 0.864769 & 2.44972 \\
\hline $4 \mathrm{mu}$ & 0.2368 & 0.835494 & 0.361526 & 0.784999 & 4.08398 & 0.911387 & 4.74151 \\
\hline $2 \mathrm{mu} 2 \mathrm{e}$ & 0.5265 & 1.0 & 0.268665 & 0.96349 & 6.76734 & 1.11862 & 7.8569 \\
\hline \multicolumn{8}{|c|}{$2_{m}^{+}(q \bar{q}) \sqrt{s}=7 \mathrm{TeV}$} \\
\hline channel & $f_{i}^{J^{P}}$ & $\alpha_{\text {ideal }}(i)$ & $\epsilon_{\text {reco }}(i)$ & $\alpha_{\exp }(i)$ & $N_{\exp }^{J^{P}}(i)$ & $\alpha_{\text {norm }}(i)$ & $N_{\text {norm }}^{J^{P}}(i)$ \\
\hline $4 \mathrm{e}$ & 0.2368 & 0.835494 & 0.180851 & 0.593713 & 0.404413 & 0.854769 & 0.582233 \\
\hline $4 \mathrm{mu}$ & 0.2368 & 0.835494 & 0.298801 & 0.636349 & 0.673168 & 0.916151 & 0.969161 \\
\hline $2 \mathrm{mu} 2 \mathrm{e}$ & 0.5265 & 1.0 & 0.24418 & 0.800531 & 1.21801 & 1.15253 & 1.75357 \\
\hline \multicolumn{8}{|c|}{$2_{m}^{+}(q \bar{q}) \sqrt{s}=8 \mathrm{TeV}$} \\
\hline $4 \mathrm{e}$ & 0.2368 & 0.835494 & 0.150986 & 0.602471 & 1.70669 & 0.867378 & 2.45712 \\
\hline $4 \mathrm{mu}$ & 0.2368 & 0.835494 & 0.284727 & 0.61795 & 3.2149 & 0.889664 & 4.6285 \\
\hline $2 \mathrm{mu} 2 \mathrm{e}$ & 0.5265 & 1.0 & 0.218591 & 0.784113 & 5.50743 & 1.12889 & 7.92905 \\
\hline \multicolumn{8}{|c|}{$2_{b}^{+} \sqrt{s}=8 \mathrm{TeV}$} \\
\hline channel & $f_{i}^{J^{P}}$ & $\alpha_{\text {ideal }}(i)$ & $\epsilon_{\text {reco }}(i)$ & $\alpha_{\exp }(i)$ & $N_{\exp }^{J^{P}}(i)$ & $\alpha_{\text {norm }}(i)$ & $N_{\text {norm }}^{J^{P}}(i)$ \\
\hline $4 \mathrm{e}$ & 0.234 & 0.81758 & 0.222087 & 0.725251 & 0.494011 & 0.869832 & 0.592493 \\
\hline $4 \mathrm{mu}$ & 0.234 & 0.81758 & 0.35873 & 0.743164 & 0.786165 & 0.891317 & 0.942889 \\
\hline $2 \mathrm{mu} 2 \mathrm{e}$ & 0.5319 & 1.0 & 0.293403 & 0.957458 & 1.45677 & 1.14833 & 1.74718 \\
\hline \multicolumn{8}{|c|}{$2_{b}^{+} \sqrt{s}=8 \mathrm{TeV}$} \\
\hline $4 \mathrm{e}$ & 0.234 & 0.81758 & 0.185353 & 0.739147 & 2.09386 & 0.886499 & 2.51128 \\
\hline $4 \mathrm{mu}$ & 0.234 & 0.81758 & 0.346648 & 0.730982 & 3.80295 & 0.876706 & 4.56109 \\
\hline $2 \mathrm{mu} 2 \mathrm{e}$ & 0.5319 & 1.0 & 0.265235 & 0.945478 & 6.64082 & 1.13396 & 7.96469 \\
\hline
\end{tabular}




\section{CHAPTER 4. HIGGS SEARCHES WITH ZZ DECAYS}

Table 4.11: Table with correction factors and event yields in the different channels of the alternative spin-2 hypotheses with high dimensional couplings arising due to lepton interference and detector effects.

\begin{tabular}{|c|c|c|c|c|c|c|c|}
\hline \multicolumn{8}{|c|}{$2_{h}^{+} \sqrt{s}=7 \mathrm{TeV}$} \\
\hline channel & $f_{i}^{J^{P}}$ & $\alpha_{\text {ideal }}(i)$ & $\epsilon_{\mathrm{reco}}(i)$ & $\alpha_{\exp }(i)$ & $N_{\exp }^{J^{P}}(i)$ & $\alpha_{\text {norm }}(i)$ & $N_{\text {norm }}^{J^{P}}(i)$ \\
\hline $4 \mathrm{e}$ & 0.2453 & 0.894726 & 0.223832 & 0.791281 & 0.538988 & 0.918012 & 0.625311 \\
\hline $4 \mathrm{mu}$ & 0.2453 & 0.894726 & 0.357244 & 0.799212 & 0.845455 & 0.927213 & 0.980862 \\
\hline $2 \mathrm{mu} 2 \mathrm{e}$ & 0.5094 & 1.0 & 0.286971 & 0.946968 & 1.44081 & 1.09863 & 1.67157 \\
\hline \multicolumn{8}{|c|}{$2_{h}^{+} \sqrt{s}=8 \mathrm{TeV}$} \\
\hline $4 \mathrm{e}$ & 0.2453 & 0.894726 & 0.188832 & 0.800725 & 2.2683 & 0.928968 & 2.63159 \\
\hline $4 \mathrm{mu}$ & 0.2453 & 0.894726 & 0.343297 & 0.793683 & 4.12916 & 0.920798 & 4.79048 \\
\hline $2 \mathrm{mu} 2 \mathrm{e}$ & 0.5094 & 1.0 & 0.259049 & 0.935098 & 6.56791 & 1.08486 & 7.61982 \\
\hline \multicolumn{8}{|c|}{$2_{h}^{-} \sqrt{s}=7 \mathrm{TeV}$} \\
\hline channel & $f_{i}^{J^{P}}$ & $\alpha_{\text {ideal }}(i)$ & $\epsilon_{\mathrm{reco}}(i)$ & $\alpha_{\exp }(i)$ & $N_{\exp }^{J^{P}}(i)$ & $\alpha_{\text {norm }}(i)$ & $N_{\text {norm }}^{J^{P}}(i)$ \\
\hline $4 \mathrm{e}$ & 0.2426 & 0.875596 & 0.205982 & 0.715726 & 0.487522 & 0.903211 & 0.615229 \\
\hline $4 \mathrm{mu}$ & 0.2426 & 0.875596 & 0.336909 & 0.749146 & 0.792493 & 0.945386 & 1.00009 \\
\hline $2 \mathrm{mu} 2 \mathrm{e}$ & 0.5148 & 1.0 & 0.26108 & 0.853431 & 1.29849 & 1.07699 & 1.63864 \\
\hline \multicolumn{8}{|c|}{$2_{h}^{-} \sqrt{s}=8 \mathrm{TeV}$} \\
\hline $4 \mathrm{e}$ & 0.2426 & 0.875596 & 0.172541 & 0.734743 & 2.08139 & 0.927209 & 2.62661 \\
\hline $4 \mathrm{mu}$ & 0.2426 & 0.875596 & 0.330978 & 0.749988 & 3.90183 & 0.946448 & 4.92392 \\
\hline $2 \mathrm{mu} 2 \mathrm{e}$ & 0.5148 & 1.0 & 0.237978 & 0.847861 & 5.95518 & 1.06996 & 7.51514 \\
\hline
\end{tabular}




\section{CHAPTER 4. HIGGS SEARCHES WITH ZZ DECAYS}

\begin{tabular}{|c|c|c|c|c|c|}
\hline$J^{P}$ model & $J^{P}$ production & expect $(\mu=1)$ & obs. $0^{+}$ & obs. $J^{P}$ & CL $_{s}$ \\
\hline $0^{-}$ & any & $2.4 \sigma(2.7 \sigma)$ & $-0.9 \sigma$ & $+3.6 \sigma$ & $0.09 \%$ \\
$0_{h}^{+}$ & any & $1.7 \sigma(1.9 \sigma)$ & $0.0 \sigma$ & $+1.8 \sigma$ & $7.1 \%$ \\
$1^{-}$ & $q \bar{q} \rightarrow X$ & $2.6 \sigma(2.7 \sigma)$ & $-1.4 \sigma$ & $+4.8 \sigma$ & $0.001 \%$ \\
$1^{-}$ & any & $2.6 \sigma(2.6 \sigma)$ & $-1.7 \sigma$ & $+4.9 \sigma$ & $0.001 \%$ \\
$1^{+}$ & $q \bar{q} \rightarrow X$ & $2.1 \sigma(2.3 \sigma)$ & $-1.5 \sigma$ & $+4.1 \sigma$ & $0.03 \%$ \\
$1^{+}$ & any & $2.0 \sigma(2.1 \sigma)$ & $-1.9 \sigma$ & $+4.5 \sigma$ & $0.01 \%$ \\
$2^{+}$ & $g g \rightarrow X$ & $1.7 \sigma(1.8 \sigma)$ & $-0.8 \sigma$ & $+2.6 \sigma$ & $1.9 \%$ \\
$2^{+}$ & $q \bar{q} \rightarrow X$ & $1.6 \sigma(1.7 \sigma)$ & $-1.6 \sigma$ & $+3.6 \sigma$ & $0.03 \%$ \\
$2_{\mathrm{m}}^{+}$ & any & $1.5 \sigma(1.5 \sigma)$ & $-1.3 \sigma$ & $+3.0 \sigma$ & $1.4 \%$ \\
$2^{+}$ & $g g \rightarrow X$ & $1.6 \sigma(1.8 \sigma)$ & $-1.2 \sigma$ & $+3.1 \sigma$ & $0.9 \%$ \\
$2^{+}$ & $g g \rightarrow X$ & $3.7 \sigma(4.0 \sigma)$ & $+1.8 \sigma$ & $+1.9 \sigma$ & $3.1 \%$ \\
$2_{h}^{-}$ & $g g \rightarrow X$ & $4.0 \sigma(4.5 \sigma)$ & $+1.0 \sigma$ & $+3.0 \sigma$ & $1.7 \%$ \\
\hline
\end{tabular}

Table 4.12: List of models used in analysis of spin-parity hypotheses corresponding to the pure states of the type noted. The expected separation is quoted for two scenarios, when the signal strength for each hypothesis is pre-determined from the fit to data and when events are generated with SM expectation for the signal yield $(\mu=1)$. The observed separation quotes consistency of the observation with the $0^{+}$model or $J^{P}$ model, and corresponds to the scenario when the signal strength is pre-determined from the fit to data. The last column quotes $\mathrm{CL}_{s}$ criterion for the $J^{P}$ model. 


\section{Chapter 5}

\section{Future Measurements}

The discovery of a Higgs-like resonance provides a new window for beyond the SM physics searches. Results presented in Section 4.2 are consistent with this resonance being the SM Higgs boson. As a result, the resonance will be referred to as a Higgs boson throughout this chapter. The development of a campaign to perform high precision measurements of Higgs properties is now a top priority. If this resonance ends up being exactly the Higgs boson described by the GWS model, this campaign will likely extend into the next generation of particle accelerators.

This chapter will discuss the logical progression of the MELA techniques which have been developed and applied in previous chapters. The use of multidimensional fits for measuring the HZZ amplitude parameters (see equation 3.7) will be expounded. Projections to high luminosity scenarios of the $H \rightarrow Z Z^{*} \rightarrow 4 \ell$ process at the LHC will be studied using both multidimensional fits and the MELA tech- 


\section{CHAPTER 5. FUTURE MEASUREMENTS}

niques will be presented. The same tools will be adapted to a future $e^{+} e^{-}$collider using $Z^{*} \rightarrow Z H \rightarrow 2 \ell 2 b$ events. Finally, speculation will be made on adapting the MELA techniques to other processes at the LHC. These techniques will constitute a framework with which a campaign of precision measurements of Higgs properties can be realized.

\subsection{Multidimensional Fits}

The use of multidimensional fits and the MELA technique for measuring model parameters are complementary methods. While multidimensional fits provide the flexibility to measure all model parameters, their use comes at the cost of simplicity; detector effects and all background processes must be described in the multidimensional space of measurables. In contrast, it is not possible to use the MELA technique for simultaneously measuring all of the HZZ model parameters, but this technique allows for kinematics to be easily described, including all detector effects, in terms of one or two observables. However, recent work [34] has shed light on methods for generalizing the MELA techniques for performing multiparameter fits.

Consider an experiment in which no background events are expected and an ideal detector is used. In this case, the analytic formulas describing differential cross sections used as inputs to the MELA discriminants can be used to directly build the 


\section{CHAPTER 5. FUTURE MEASUREMENTS}

likelihood for fitting model parameters,

$$
\mathscr{L}=\Pi_{i}^{N} \mathscr{P}_{\text {sig }}\left(\vec{x}_{i} ; \vec{\xi}\right)
$$

where $\mathscr{P}$ represents the differential cross section, $\vec{x}_{i}$ are the observables for event i, and $\vec{\xi}$ are the model parameters for which the likelihood will be maximized with respect to. For multidimensional fits, $\vec{x}_{i}$ represents the set of masses and angular decay variables: $m_{1}, m_{2}, \cos \theta^{*}, \cos \theta_{1}, \cos \theta_{2}, \Phi$, and $\Phi_{1}$. For fits done with the MELA technique, $\vec{x}_{i}$ represents one or more discriminants which have been particularly chosen for a specific fit.

Fits done with the multidimensional likelihood can be computationally efficient, if the analytical integral of the likelihood can be provided for all points in the parameter space. For example, the $H \rightarrow Z Z^{*} \rightarrow 4 \ell$ analysis at the LHC makes use of 8 observables which distinguish different scalar models and background. If one were to attempt to measure each of the four model parameters simultaneously, either the $8 \mathrm{D}$ integral should be known a priori at each point in the $4 \mathrm{D}$ parameter space or numerical integration over the 8 observables must be performed at each point in the 4D parameter space. The latter is nearly impossible.

By comparing the effectiveness of both, these complementary methods provide a powerful resource for cross-checking and validating each other. Together, they provide a framework for exploring new methods for constraining Higgs properties. 


\section{CHAPTER 5. FUTURE MEASUREMENTS}

For the ideal distributions, it is possible to calculate the integral of the likelihood analytically as a function of the 4 model parameters and this has been done for the $H \rightarrow Z Z^{*} \rightarrow 4 \ell$ process. Using the the likelihood presented above, toy studies can be performed to compare the precision of measuring $f_{a 3}$ using either multidimensional fits or the MELA technique. Figure 5.2 shows the results of three types of fits: multidimensional fits in which $f_{a 3}$ is floated, multidimensional fits in which $f_{a 3}$ and $\phi_{a 3}$ are floated, and 1D fits using the MELA technique floating $f_{a 3}$.

In all three cases, toys generated correspond to a scalar resonance with $f_{a 3}=0.18$. The results of the 1D fit and the 5D, 2 parameter fit are both compatible. However, it is found that the 1 parameter multidimensional fit provides a $4 \%$ improvement. For generated values of $f_{a 3}=0.06$ and 0.02 this improvement is found to be $13 \%$ and $30 \%$, respectively. The interpretation of this is that the relative importance of interference terms in Equation 3.6, which is not accounted for in the MELA technique, becomes large for small values of $f_{a 3}$.

The two examples of multidimensional fits shown in Figure 5.2 are two different ways of interpreting data. When $\phi_{a 3}$ is floated, this parameters is in principle being profiled, reducing the expected precision due to the lack of prior knowledge of the phase. Fits done using the MELA technique are insensitive to the kinematics effects of $\phi_{a 3}$ and thus are equivalent to profiling this parameter. In contrast, one can argue that all anomalous couplings should be real if the assumption that there are no light particles which can induce effective couplings through loop diagrams is made. In this 


\section{CHAPTER 5. FUTURE MEASUREMENTS}

case, one can assume prior knowledge of the phase and fix $\phi_{a 3}$ to zero. The equivalent measurement using the MELA techniques can be made if the standard methods are extended. This minimally relies on modifying the likelihood parameterization to include interference effects. This can done by replacing Equation 3.9 with

$$
\begin{array}{r}
\mathscr{P}\left(\vec{x} \mid f_{a 3}, \phi_{a 3}\right)=\left(1-f_{a 3}\right) \mathscr{P}_{0^{+}}(\vec{x})+f_{a 3} \mathscr{P}_{0^{-}}(\vec{x})+ \\
\sqrt{f_{a 3}\left(1-f_{a 3}\right)}\left[\mathscr{P}_{i n t}\left(\vec{x} \mid \phi_{a 3}=0\right) \cos \phi_{a 3}+\mathscr{P}_{i n t}\left(\vec{x} \mid \phi_{a 3}=\pi / 2\right) \sin \phi_{a 3}\right]
\end{array}
$$

where $\vec{x}$ corresponds to any set of discriminant variable and $\mathscr{P}_{\text {int }}$ is the distribution of the interference portion of the differential cross section assuming either $\phi_{a 3}=0$ or $\phi_{a 3}=\pi / 2$. This parameterization can also be used to measure $f_{a 2}$ whithin the MELA framework. The use of aditional discriminants can be used to increase sensitivity to kinematic differences caused by interference. For example, to increase sensitivity to $f_{a 3}$ measurements, the additional variable would be

$$
D_{C P}=\frac{\mathscr{P}_{i n t}\left(m_{1}, m_{2}, \vec{\Omega} \mid \phi_{a 3}=0\right)}{\mathscr{P}_{0^{+}}\left(m_{1}, m_{2}, \vec{\Omega}\right)+\mathscr{P}_{0^{-}}\left(m_{1}, m_{2}, \vec{\Omega}\right)}
$$

Analogously, interference effects relevant to $f_{a 2}$ measurements can be accounted for using

$$
D_{i n t}=\frac{\mathscr{P}_{i n t}\left(m_{1}, m_{2}, \vec{\Omega} \mid \phi_{a 2}=0\right)}{\mathscr{P}_{0^{+}}\left(m_{1}, m_{2}, \vec{\Omega}\right)+\mathscr{P}_{0_{h}^{+}}\left(m_{1}, m_{2}, \vec{\Omega}\right)}
$$

Figure 5.1 shown examples of $D_{\text {int }}$ distributions for measuring either $f_{a 3}$ or $f_{a 2}$. Using $\vec{x}=\left\{D_{0^{-}}, D_{\text {int }}\right\}\left(\vec{x}=\left\{D_{0^{-}}, D_{\text {int }}\right\}\right)$ in conjuction will Formula 5.2 allows for sensitivity 


\section{CHAPTER 5. FUTURE MEASUREMENTS}
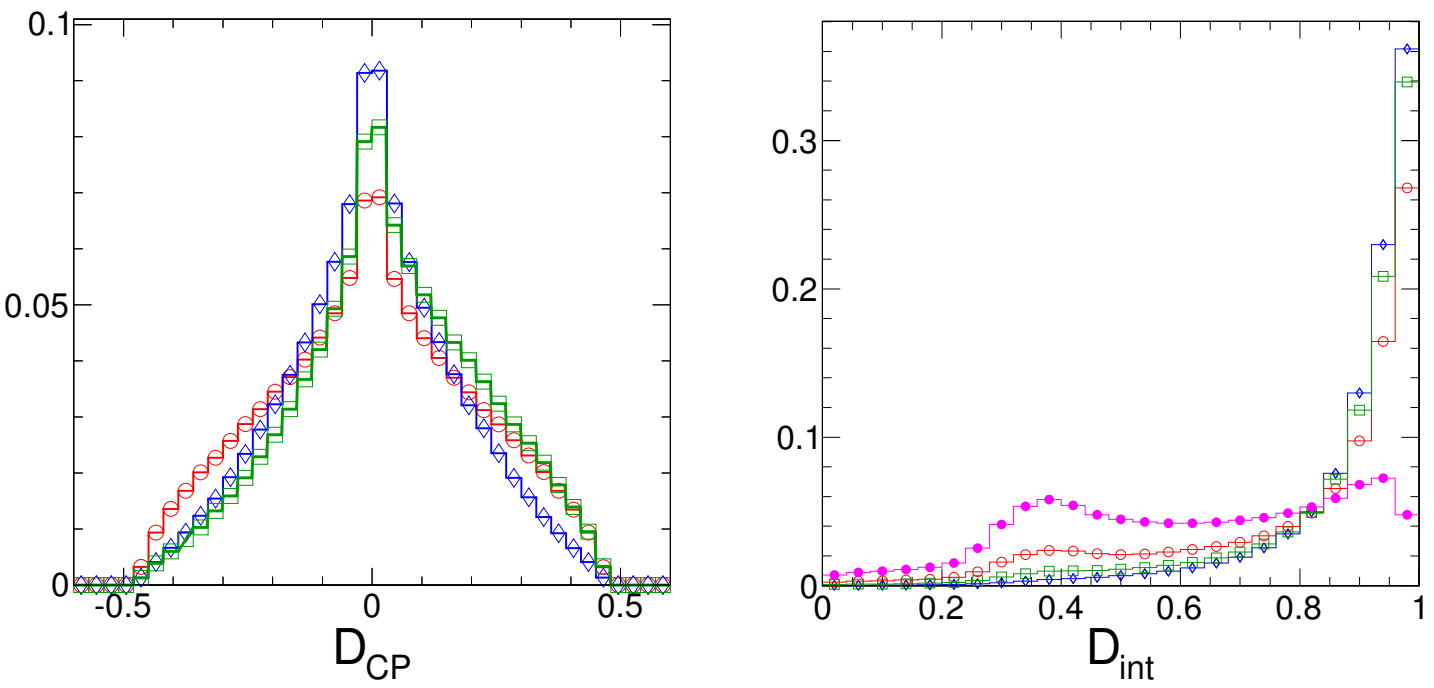

Figure 5.1: Distributions of $D_{C P}$ (right) and $D_{\text {int }}$ (left) are shown for several scalar models. Distributions for a SM Higgs are respresented by red circles, pure alternative scalar models (either $0^{-}$or $0_{h}^{+}$) by blue diamonds, and mixed scalar models corresponding to $f_{a 3}=0.5$ and $f_{a 2}=0.5\left(\phi_{a i}=0\right)$ for left and right plots, respectively by green squares. The closed magenta circles in the right plot corresponds to a mixed scalar models with $f_{a 2}=0.5$ and $\phi_{a 2}=\pi$. 


\section{CHAPTER 5. FUTURE MEASUREMENTS}

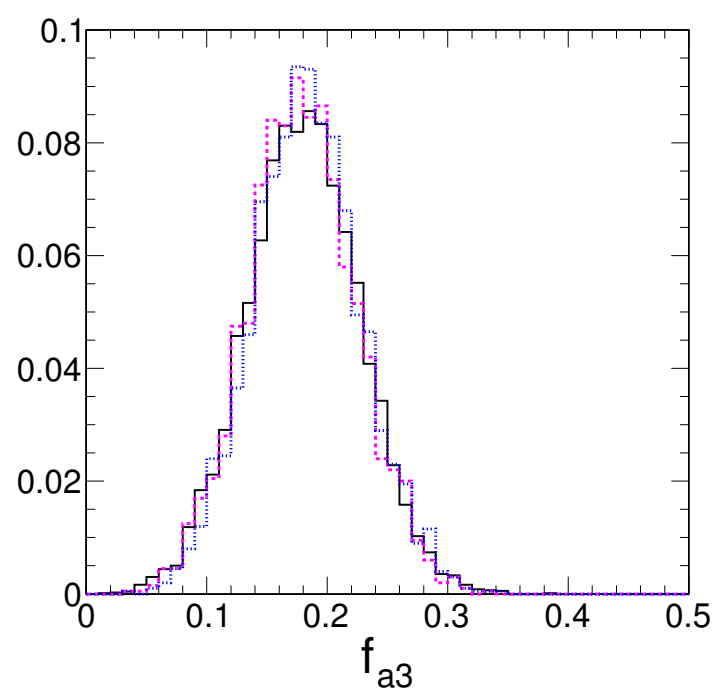

Figure 5.2: Distribution of best-fit $f_{a 3}$ values from a large number of generated experiments using either the $1 \mathrm{D}$ fit of the $D_{0^{-}}$distributions (solid black), 7D fits with only $f_{a 3}$ unconstrained (dashed magenta), or $7 \mathrm{D}$ fits with $f_{a 3}$ and $\phi_{a 3}$ unconstrained (dotted blue).

due to interference effects on both relative normalization and kinematic distributions to be recovered. Constraining the relative phase, $\phi_{a 3}$ or $\phi_{a 2}$, requires an additional discriminant which take into account kinematics from complex phases,

$$
\begin{aligned}
D_{C P}^{\perp} & =\frac{\mathscr{P}_{i n t}\left(m_{1}, m_{2}, \vec{\Omega} \mid \phi_{a 3}=\pi / 2\right)}{\mathscr{P}_{0^{+}}\left(m_{1}, m_{2}, \vec{\Omega}\right)+\mathscr{P}_{0^{-}}\left(m_{1}, m_{2}, \vec{\Omega}\right)} . \\
D_{i n t}^{\perp} & =\frac{\mathscr{P}_{i n t}\left(m_{1}, m_{2}, \vec{\Omega} \mid \phi_{a 2}=\pi / 2\right)}{\mathscr{P}_{0^{+}}\left(m_{1}, m_{2}, \vec{\Omega}\right)+\mathscr{P}_{0^{+}}\left(m_{1}, m_{2}, \vec{\Omega}\right)} .
\end{aligned}
$$

Validations of these types of measurements using more than one discriminant for measuring one or more parameters is presented in more detail in reference [34]. 


\section{CHAPTER 5. FUTURE MEASUREMENTS}

\subsection{LHC Projections}

As a point of reference, the expected precision for measuring $f_{a 3}\left(=f_{g 4}\right)$ that CMS can reach in the $H \rightarrow Z Z^{*} \rightarrow 4 \ell$ analysis is estimated using both multidimensional fits and the MELA technique. Detector simulations are modeled by including finite momentum and angular resolution of lepton four vectors and applying analysis selections both of which are meant to roughly mimic the CMS public analysis [86]. Leptons are required to have $|\eta|<2.4, p_{T}>5 \mathrm{GeV}$, and $m_{2}>12 \mathrm{GeV}$. The resolution effects result in a $m_{4 \ell}$ width of approximately $2 \mathrm{GeV}$, similar to that of the $2 e 2 \mu$ channel.

Two luminosity scenarios are tested, $300 \mathrm{fb}^{-1}$ and $3000 \mathrm{fb}^{-1}$. Shapes are modeled using ideal MC simulations with the approximate detector effects described above. Background shapes are taken purely from POWHEG simulation of $q \bar{q} \rightarrow Z Z^{*} \rightarrow 2 e 2 \mu$ events. Signal shapes are taken purely from JHUGen simulation of $g g \rightarrow H \rightarrow Z Z^{*} \rightarrow$ $2 e 2 \mu$ events. The number of events expected for signal and background are listed in Table 5.2 and are based on results from Chapter 4.2 .

In the case of multidimensional fits, some approximations are used for modeling the distribution of background events and for modeling the distribution of signal events with detector effects on signal distributions. Both acceptance and resolution effects are modeled as uncorrelated multiplicative corrections to the ideal signal distributions. The background is modeled as a fully uncorrelated set of distribution for each of the individual measurables. The projections for both signal and background events, before and after detector effects are shown in Figure 5.3. Although these 


\section{CHAPTER 5. FUTURE MEASUREMENTS}

\begin{tabular}{ccccc}
\hline \hline energy & $\int \mathscr{L} d t\left[f b^{-1}\right]$ & $\sigma \times \mathscr{B}\left[f b^{-1}\right]$ & $N_{\text {prod }}$ & $N_{\text {reco }}$ \\
\hline \hline \multicolumn{5}{c}{$p p \rightarrow H \rightarrow Z Z^{*} \rightarrow 4 \ell$} \\
\hline $14 \mathrm{TeV}$ & 300 & 6.23 & 18694 & 5608 \\
\hline \multicolumn{5}{c}{$p p \rightarrow Z Z^{*} \rightarrow 4 \ell$} \\
\hline $14 \mathrm{TeV}$ & - & - & 2243 \\
\hline $250 \mathrm{GeV}$ & $e^{+} e^{-} \rightarrow Z^{*} \rightarrow Z H \rightarrow 2 \ell 2 b$ \\
\hline \multicolumn{5}{c}{9.35} \\
\hline $250 \mathrm{GeV}$ & $e^{+} e^{-} \rightarrow Z Z \rightarrow 2 \ell 2 b$ & 2337 & 1870 \\
\hline \hline
\end{tabular}

Table 5.1: List of cross sections and event yields for Higgs production and decay processes.

approximations cause small biases in toy studies, they provide a description which is accurate enough to estimate the precision of such measurements using toys generated directly from probability density functions.

The distribution of fitted $f_{a 3}$ values are shown in Figure 5.4. The precision of $f_{a 3}$ measurements using multidimensional fits is found to be similar as those estimated from 1D fits. It is estimated that CMS will have sufficient sensitivity for at least a $3 \sigma$ discovery of $\mathrm{CP}$-violating interactions in the $H \rightarrow Z Z^{*}$ channel for values of $f_{a 3} \geq 0.18(0.06)$ with $300(3000) f b^{-1}$, respectively. Using multidimensional fits, it is estimated that CMS can also achieve sufficient sensitivity for a $3 \sigma$ or better discovery of anomalous CP-even couplings for values of $f_{a 2} \geq 0.14$ (0.088) with 300 (3000) $\mathrm{fb}^{-1}$, respectively. 


\section{CHAPTER 5. FUTURE MEASUREMENTS}
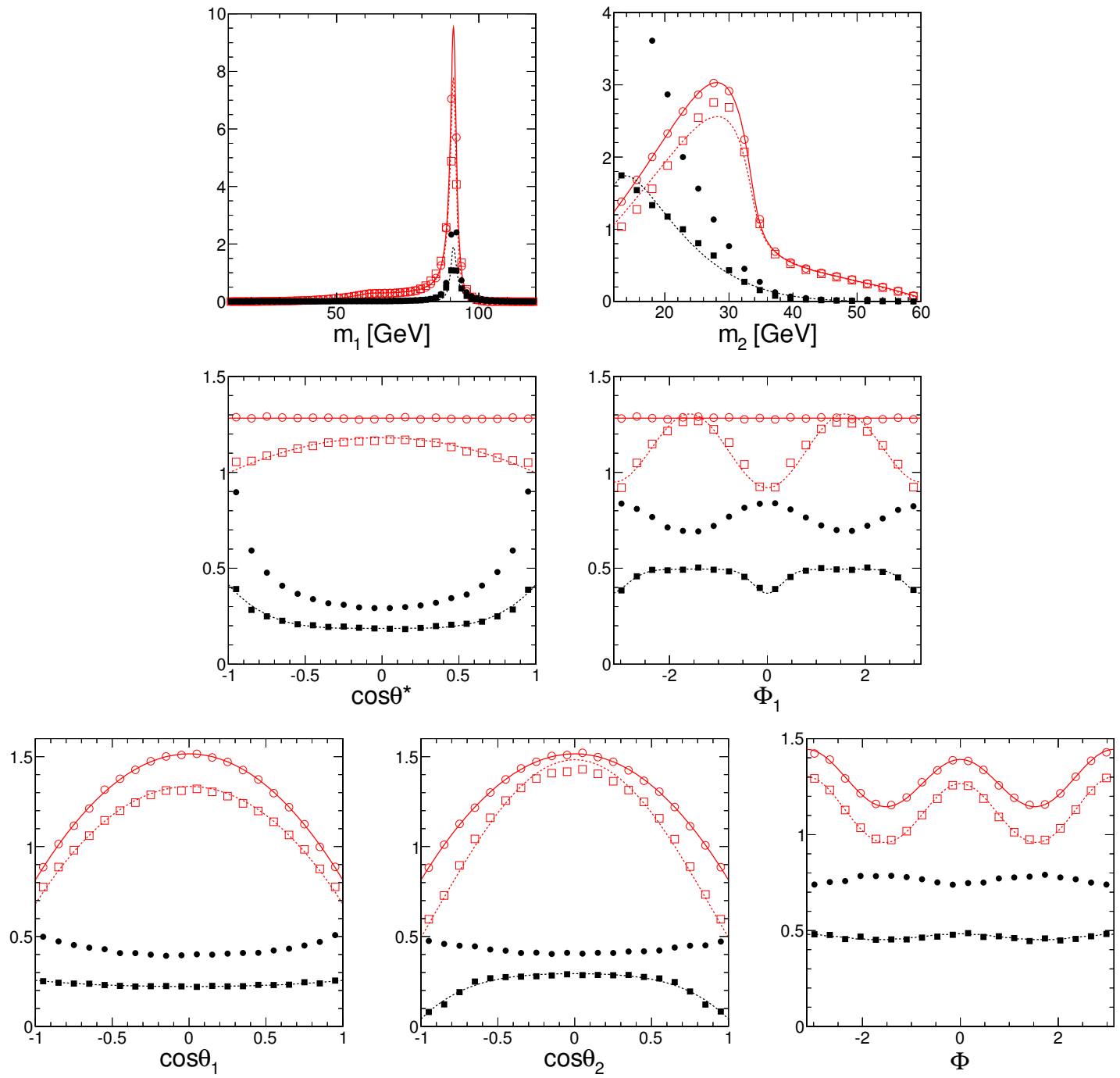

Figure 5.3: Distributions of masses (top row), production angles (middle row), and helicity angles (bottom row), in the $H \rightarrow Z Z^{*} \rightarrow 4 \ell$ analysis at the LHC. Open red points show simulated events for the SM Higgs boson with curves showing projections of analytical distributions. Solid black points show background distributions with curves showing projections of analytical parametrization. Distributions before (circles) and after (squares) detector effects are shown. 


\section{CHAPTER 5. FUTURE MEASUREMENTS}

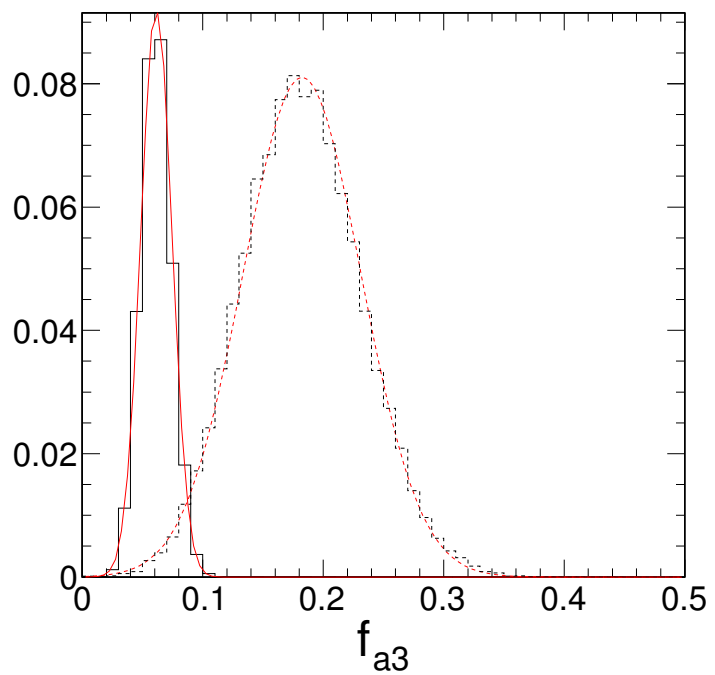

Figure 5.4: Distributions of fitted values of $f_{a 3}$ from a large number of generated experiments in the $H \rightarrow Z Z^{*} \rightarrow 4 \ell$ channel at the LHC. Results for the $300 \mathrm{fb}^{-1}$ (dotted) and $3000 \mathrm{fb}^{-1}$ (solid) scenarios are shown.

\subsection{Future Colliders}

Similar measurements can be made with other processes such as $e^{+} e^{-} \rightarrow Z^{*} \rightarrow$ $Z H \rightarrow 2 \ell 2 b$. The diagrams in Figure 5.5 demonstrate that this process is equivalent to the $p p \rightarrow H \rightarrow Z Z \rightarrow 4 \ell$ process, except it probes a different region of phase space. Thus, the differential cross sections presented in Section 3 are all still applicable. The 


\section{CHAPTER 5. FUTURE MEASUREMENTS}

probability distribution is given by equation 3.5 where

$$
\begin{array}{r}
\frac{d \Gamma \text { }=0}{\Gamma d \vec{\Omega}}=4\left|A_{00}^{2}\right| \sin ^{2} \theta_{1} \sin ^{2} \theta_{2} \\
+\left|A_{++}\right|^{2}\left(1-2 R_{1} \cos \theta_{1}+\cos ^{2} \theta_{1}\right)\left(1+2 A_{f 2} \cos \theta_{2}+\cos ^{2} \theta_{2}\right) \\
+\left|A_{--}\right|^{2}\left(1+2 R_{1} \cos \theta_{1}+\cos ^{2} \theta_{1}\right)\left(1-2 A_{f 2} \cos \theta_{2}+\cos ^{2} \theta_{2}\right) \\
-4\left|A_{00}\right|\left|A_{++}\right|\left(R_{1}-\cos \theta_{1}\right) \sin \theta_{1}\left(A_{f 2}+\cos \theta_{2}\right) \sin \theta_{2} \cos \left(\Phi+\phi_{+0}\right) \\
-4\left|A_{00}\right|\left|A_{--}\right|\left(R_{1}+\cos \theta_{1}\right) \sin \theta_{1}\left(A_{f 2}-\cos \theta_{2}\right) \sin \theta_{2} \cos \left(\Phi-\phi_{-0}\right) \\
+2\left|A_{++}\right|\left|A_{--}\right| \sin ^{2} \theta_{1} \sin ^{2} \theta_{2} \cos \left(2 \Phi-\phi_{-0}-\phi_{+0}\right),
\end{array}
$$

$A_{i, j}$ is given by Formula $3.3, R_{1}=\left(A_{f 1}+P^{-}\right) /\left(1+A_{f 1} P^{-}\right), A_{f i}=2 \bar{g}_{V}^{f} \bar{g}_{A}^{f} /\left(\bar{g}_{V}^{f 2}+\right.$ $\bar{g}_{A}^{f 2}$ ) is the parameter characterization the decay $Z_{i} \rightarrow f_{i} \bar{f}_{i}$, and $P^{-}$is the effective polarization of the electron beam defined such that $P^{-}=0$ corresponds to unpolarized beams. In the translation from the different coupling parametrizations in Equation 3.1 and Equation 3.2, s should be negated. For this process, the Z boson and Higgs boson are both on-shell and their mass can be approximated as constant. Thus, three nontrivial angular distributions describe the kinematics of this process. Figure 5.6 shows the ideal angular distributions for several scalar models: SM Higgs, a pseudoscalar, and two mixed parity scalar models with phases $\phi_{3}=0, \pi / 2$.

Note, the equivalent $f_{a 3}$ parameter for this process will have slightly different meaning. For example, Table 5.2 summarizes how the value for $f_{a 3}$ of the $H \rightarrow Z Z^{*}$ process can be translated. The numbers in this table reflect the fact that the ratio 


\section{CHAPTER 5. FUTURE MEASUREMENTS}
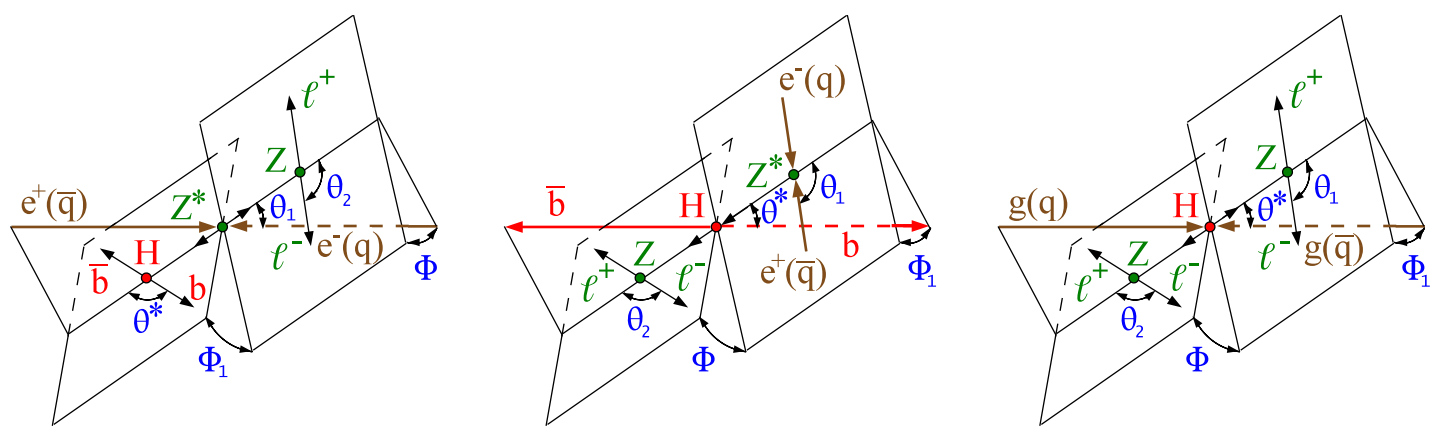

Figure 5.5: Diagrams showing the different processes produced via the HZZ amplitude. The $e^{+} e^{-} \rightarrow Z^{*} \rightarrow Z H \rightarrow 2 \ell 2 b$ process in the $Z^{*}$ and $\mathrm{H}$ rest frame are shown in the left and middle plot, respectively. The $p p \rightarrow H \rightarrow Z Z^{*} \rightarrow 4 \ell$ process is shown in the $\mathrm{H}$ rest frame is shown in the right plot.
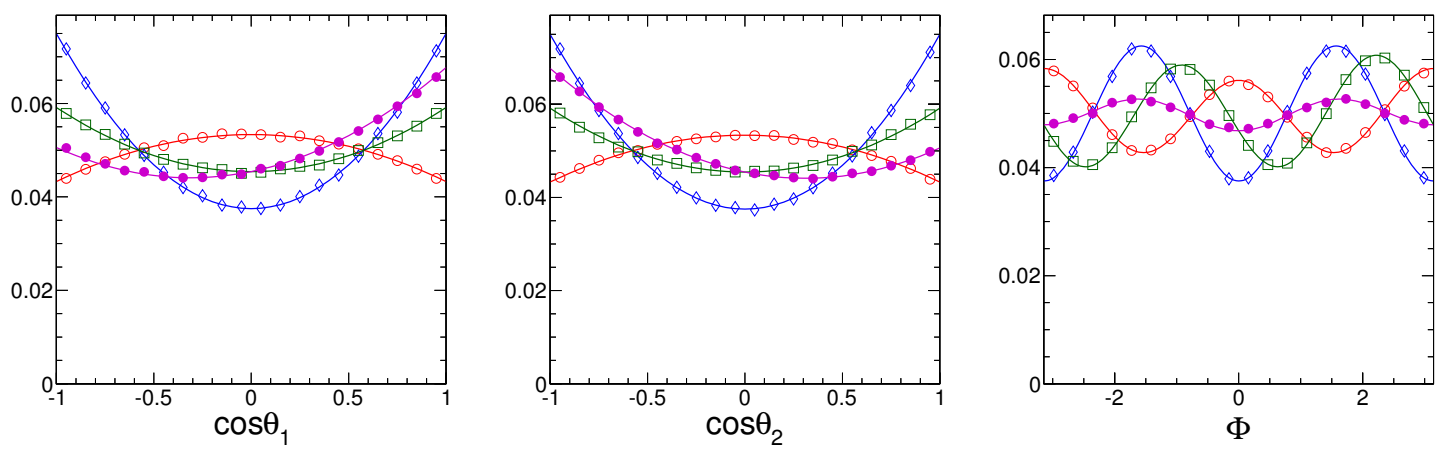

Figure 5.6: Angular distributions, $\cos \theta_{1}$ (left), $\cos \theta_{2}$ (middle), and $\Phi$ (right), of four different scalar models of the process $e^{+} e^{-} \rightarrow Z^{*} \rightarrow Z H$. Markers show angular distributions from simulations while lines show projections of the angular distributions presented in Section 3. Red line/circles represent a SM Higgs, blue lines/diamonds represent a pseudoscalar, green lines/squares and purple lines/solid circles represent a mixed parity scalar $\left(f_{a 3}=0.1\right)$ with various phases. 


\section{CHAPTER 5. FUTURE MEASUREMENTS}

\begin{tabular}{l|l}
$g 1 / g 4$ & 0.85 \\
\hline \hline$f_{a 3}^{\left(H \rightarrow Z Z^{*}\right)}$ & 0.10 \\
$f_{a 3}^{(q \bar{q} \rightarrow Z H)}$ & 0.81 \\
$f_{a 3}^{(q \bar{q} \rightarrow H q \bar{q})}$ & 0.93 \\
$f_{a 3}^{\left(e^{+} e^{-} \rightarrow Z H\right)}(\sqrt{s}=250)$ & 0.85 \\
$f_{a 3}^{\left(e^{+} e^{-} \rightarrow Z H\right)}(\sqrt{s}=500)$ & 0.99 \\
\hline \hline
\end{tabular}

Table 5.2: List of $f_{a 3}$ values for various processes.

$\sigma_{1} / \sigma_{4}$, as defined in Section 3, can vary by orders of magnitude between different processes. Larger $f_{a 3}$ values correspond to having effectively more events which look like a pseudoscalar. As a result, the sensitivity to CP-violating interactions is expected to be larger for other processes.

Similar to the $H \rightarrow Z Z^{*}$ analysis, a kinematic discriminant built according to equation 4.1 can be used to measure $f_{a 3}$ according to equation 3.9. Toy studies have been done to justify that there are no biases introduced by the approximations in equation 3.9 .

Projections for a future $e^{+} e^{-}$collider are estimated assuming a collision energy of $250 \mathrm{GeV}$ and an integrated luminosity of $250 \mathrm{fb}^{-1}$. Signal events are simulated with JHUGen. Background events are modeled using $e^{+} e^{-} \rightarrow Z Z$ events simulated with MADGRAPH. The cross sections and event yields for the signal and background processes are detailed in Table 5.2 which are based on previous studies in references $[90,91]$.

All events are required to have two leptons whose transverse momentum is greater 


\section{CHAPTER 5. FUTURE MEASUREMENTS}
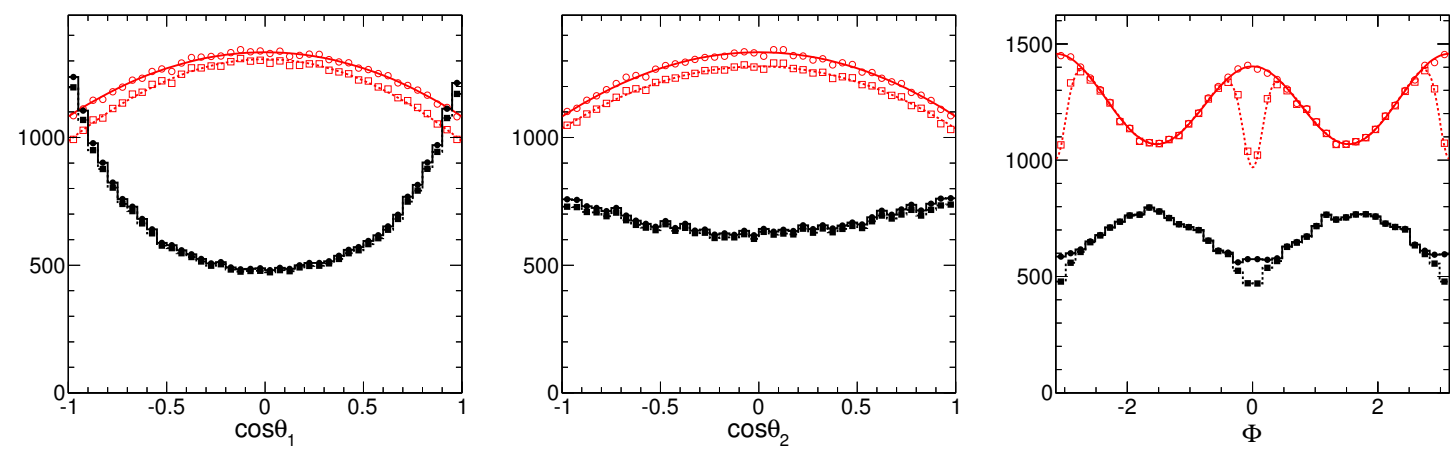

Figure 5.7: Expected distribution of three helicity angles for a SM Higgs boson (red) and the SM background (black) before (solid lines) and after (dashed lines) acceptance cuts.

than $5 \mathrm{GeV},|\eta|<2.4$, and Higgs boson mass between $115<m_{H}<140 \mathrm{GeV}$. Although the background process is not fully representative of the expected backgrounds that will exist in $e^{+} e^{-}$collisions, the exact modeling of background events is not critical for the purposes of this study. The distribution of signal and background events and the effect of acceptance cuts are shown for each of the three angles in Figure 5.7.

Similarly to before, toys are generated and fit using Equation 3.9. The distribution of the best-fit $f_{a 3}$ for a signal model corresponding to $f_{a 3}=0.1$ is shown in the left plot of Figure 5.8. The expected precision is found to be $\sigma_{f_{a 3}}=0.04$. Converting this to the $f_{a 3}$ parameter currently being measured at the LHC, $f_{a 3}^{d e c}\left(H \rightarrow Z Z^{*}\right)$, the error on this parameter is found to be $\sigma_{f_{a 3}^{d e c}}=0.0008$. This result can be compared to the LHC scenario where the error for the high luminosity scenario was $\sigma_{f_{a 3}^{d e c}} \sim 0.03$. 


\section{CHAPTER 5. FUTURE MEASUREMENTS}

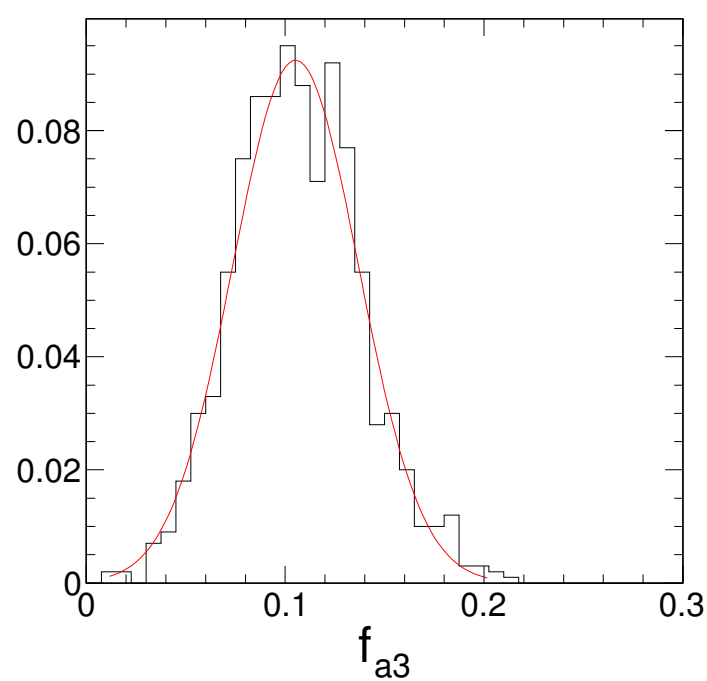

Figure 5.8: Distribution of the best-fit value of $f_{a 3}$ from a large number of generated experiments. Toys were generated using a value of $f_{a 3}=0.1$.

\subsection{Other Channels}

The sensitivity to CP-violating interactions in the HZZ amplitude is markedly better using $e^{+} e^{-}$collisions. This is due to the fact that the $\sigma_{4} / \sigma_{1}$ in equation 3.7 can be much larger when $\mathrm{Z}$ bosons are produced far off shell. However, it should be noted that this simple exercise does not completely diminish the potential for similar measurements at the LHC. Other processes at the LHC shown in Table 5.2, e.g. $q \bar{q} \rightarrow H+q \bar{q}$ and $q \bar{q} \rightarrow Z^{*} \rightarrow Z H$, also benefit from enhanced $\sigma_{4}$ due to the isolated phase space that they probe. As these channels continue to gain sensitivity to signal events, they will play an increasingly important role in constraining anomalous couplings of HZZ interactions. Detailed studies are still to be done, but these channels may ultimately dominate the precision of $f_{a 3}$ measurements at the LHC. 


\section{CHAPTER 5. FUTURE MEASUREMENTS}

\subsection{Summary}

There are several complications involved with applying multidimensional fits to the $H \rightarrow Z Z^{*}$ or other processes: modeling a multidimensional transfer function appropriate to event reconstruction and analysis selections; describing all backgrounds accurately; and building likelihoods which can be efficiently minimized. However, multidimensional fits provide a flexible approach which could ultimately measure each of the model parameters which describe the HZZ amplitude.

A number of the challenges related to multidimensional fits can mitigated by using the MELA technique, discussed in chapter 3 and applied in Section 4.2. These techniques help largely because the problem is reduced from using many observables to using at most a couple of observables. As with multidimensional fits, these techniques are applicable to more processes than just $H \rightarrow Z Z^{*}$.

Current measurements being done at CMS to constrain CP-violating interactions are only making use of $H \rightarrow Z Z^{*}$ events. Similar measurements can be made using $Z^{*} \rightarrow Z H$ events at an $e^{+} e^{-}$collider. The estimated precision on $f_{a 3}$ that can be

expected at an $e^{+} e^{-}$collider is found to be $\sigma_{f_{a 3}}^{d e c} \sim 0.0008$, which is several orders of magnitude better than the estimated precision on $f_{a 3}$ at the $\mathrm{LHC}$ using $H \rightarrow Z Z^{*} \rightarrow$ $4 \ell$ events with $3000 \mathrm{fb}^{-1}, \sigma_{f_{a 3}}^{\text {dec }} \sim 0.03$. However, it is likely that other channels at the LHC will one day probe much larger regions of the parameters space. Ultimately, these tools may become a staple of Higgs property measurements for many years. 


\section{Chapter 6}

\section{Conclusions}

A set of analysis tools which can be used to enhance the sensitivity of diboson signatures as well as to study resonance properties have been developed. Two specific implementations of these tools have been presented in the context of searches for a Higgs boson.

A search for a SM Higgs boson using $Z Z^{(*)} \rightarrow 2 \ell 2 q$ events was presented. Drawing on the ideas presented in Chapter 3, a novel discriminant was used to reduce the dominant SM background. Techniques for measuring expected background shapes and event yields using data control regions were used. No significant deviation from the background only hypothesis was found and upper limits were set. Standard Model Higgs boson masses between 340 and $390 \mathrm{GeV}$ were ruled out at $95 \%$ confidence level.

A search for a SM Higgs boson using $Z Z^{(*)} \rightarrow 4 \ell$ events was presented. Again, ideas from Chapter 3 were used to build discriminants to further enhance sensitivity 


\section{CHAPTER 6. CONCLUSIONS}

to signal events. These techniques have been an integral part of the $Z Z \rightarrow 4 \ell$ analysis at CMS since the discovery of the Higgs-like resonance in July of 2012. Now, an excess of events is observed with a local significance of $6.8 \sigma$ at $125.7 \mathrm{GeV}$. At other masses, no significant excesses were observed and Higgs boson masses in the range [114.5,119] and $[129-800]$ were ruled out at $95 \%$ confidence level.

Other MELA discriminants were designed to test the compatibility of the excess in data with respect to either a SM Higgs boson or a number of signal models. All tests show that data prefers the SM Higgs hypothesis over the alternative hypotheses. Most notably data disfavors the pseudoscalar model at the level of $0.04 \%$. These property measurements are summarized in Figure 6.1. The contributions of CPviolating interactions were constrained through the measurement of $f_{a 3}$. The best-fit value of this parameter is found to be $f_{a 3}=0.00_{-0.00}^{+0.17}$ which is consistent with SM expectation. The $95 \%$ confidence interval of this parameter is found to be $[0.00,0.51]$.

Hypothesis separation measurements were also performed using WW events for testing the minimal coupling graviton model. This result has been combined with the ZZ result by performing simultaneous fits in both channels [92]. The result is shown in Figure 6.2. The median of the SM Higgs toy distribution has a $C L_{s}$ value of $1.25 \%$, corresponding to an average separation of $3.0 \sigma$. The data is found to disfavor the minimal coupling graviton with a $C L_{s}$ value of $0.6 \%$, compared to the observed $C L_{s}$ of $1.3 \%^{1}$ and $6.8 \%$ using the $\mathrm{ZZ}$ and WW channels alone. Other measurements

\footnotetext{
${ }^{1}$ Note that this results corresponds to an earlier version of the analysis [86]. The most up to date ZZ/WW combination does not exist yet.
} 


\section{CHAPTER 6. CONCLUSIONS}

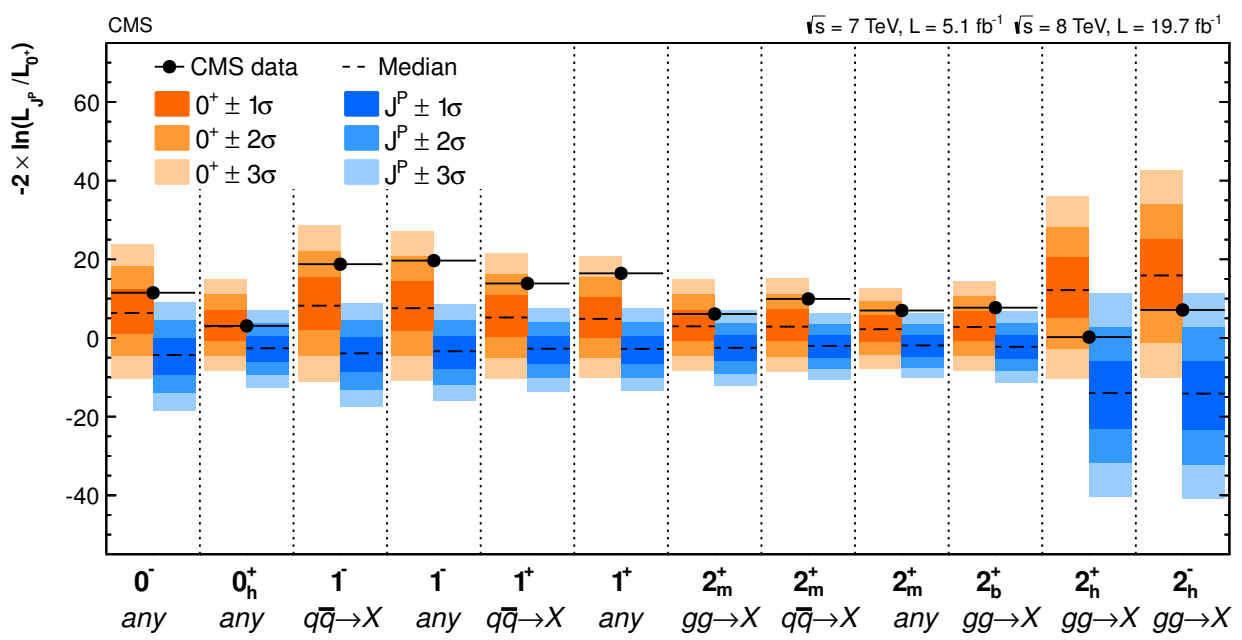

Figure 6.1: Distribution of test statistics for SM Higgs toys (blue), alternative $J^{P}$ signals toys (orange), and the observed test statistic (points).

performed by the ATLAS collaboration $[93,94]$ using the same ideas developed in Chapter 3 are consistent with those presented in Section 4.2.

Cross section measurements in other channels also support the SM Higgs hypothesis [92]. The left plot of Figure 6.3 shows the best-fit signal strength of each decay channel separately. The best-fit signal for different production mechanisms is shown in the right plot of Figure 6.3. All are consistent with the the SM Higgs hypothesis, $\mu=1$. As described in Chapter 3, it is expected that the fermionic couplings to the Higgs field will scale with the mass of the fermion while the bosonic couplings to the Higgs field will scale with the square of the vector boson's mass. Figure 6.4 shows the best-fit fermionic coupling and the square-root of the bosonic couplings divided by twice the Higgs vacuum expectation value. All couplings measured thus far are consistent with a linear correlation between the couplings and the masses. 


\section{CHAPTER 6. CONCLUSIONS}

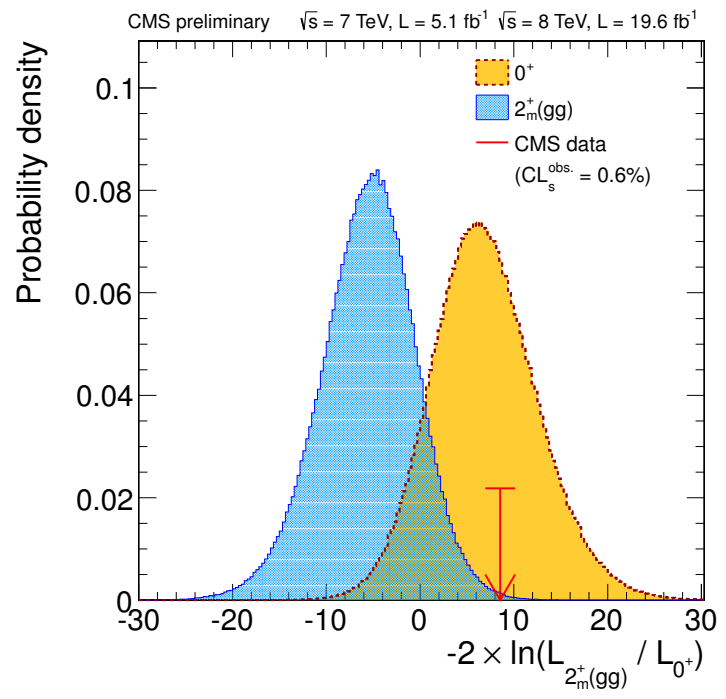

Figure 6.2: Distributions of the test statistic comparing the SM Higgs hypothesis against the $J^{P}=2_{m}^{+}$hypothesis using a simultaneous fit of the signal strength in the ZZ and WW channels. The orange distribution represents the SM Higgs toys, the blue distribution represents the $2_{m}^{+}$hypothesis. The red arrow shows the observed test statistic.

The measurements discussed above strongly suggest that the resonance observed is a scalar which participates in electroweak symmetry breaking. Extensions to the SM which fall under the generic class of $2 \mathrm{HDM}$ provide an interesting framework to further study the Higgs sector. These models predict two more neutral scalar bosons and could lead to CP-violating interactions. As discussed in Chapter 1, this could help to explain the baryon asymmetry problem or even dark matter if the specific 2HDM turns out to be SUSY.

Although CMS measurements have begun to constrain the presence of CP-violating interactions by setting limits on $f_{a 3}\left(f_{g 4}\right)$, these measurements still have large uncertainties. However, the same tools which are currently being used in the $H \rightarrow Z Z$ 


\section{CHAPTER 6. CONCLUSIONS}
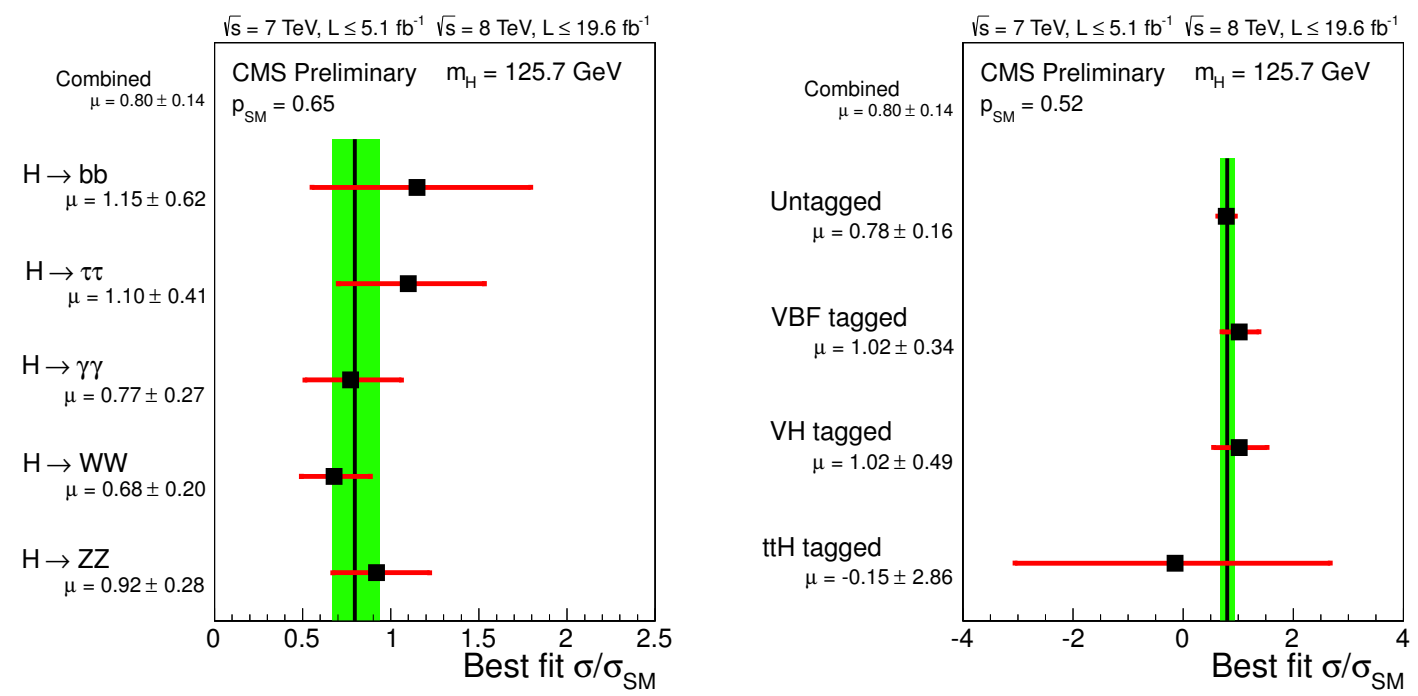

Figure 6.3: Best-fit signal strength modifier, $\mu$, for various production and decay modes. Red error bars represent the $68 \%$ confidence interval of the individual measurements. Black lines represent the combined measurement of all channels (production and decay); the green band represents the the $68 \%$ confidence interval. All fits are done for a fixed mass hypothesis, $m_{H}=125.7 \mathrm{GeV}$, which correspond to the combined best-fit value. 


\section{CHAPTER 6. CONCLUSIONS}

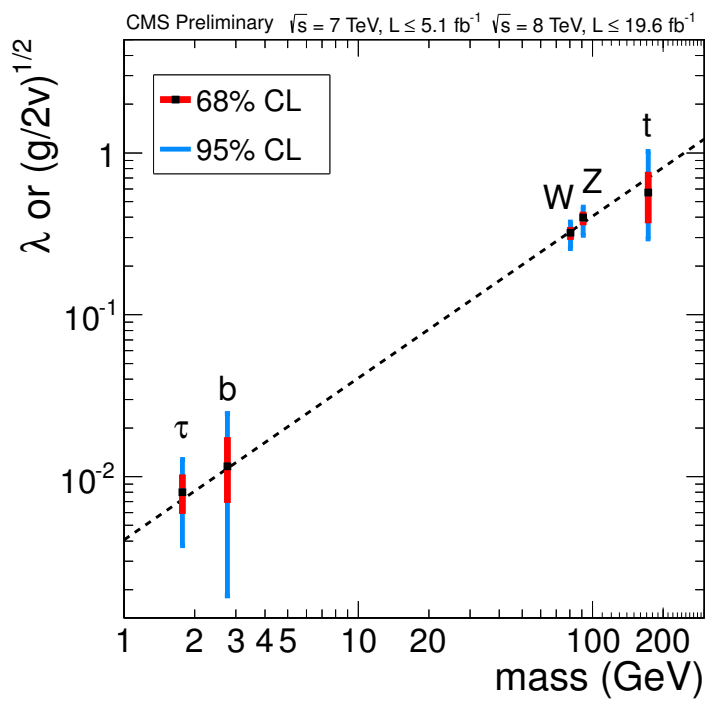

Figure 6.4: Summary of the fits for deviations in the coupling for the generic fiveparameter model not including effective loop couplings, expressed as function of the particle mass. For the fermions, the values of the fitted Yukawa couplings hff are shown, while for vector bosons the square-root of the coupling for the hVV vertex divided by twice the vacuum expectation value of the Higgs boson field. Particle masses for leptons and weak boson, and the vacuum expectation value of the Higgs boson are taken from the PDG. For the top quark the same mass used in theoretical calculations is used $(172.5 \mathrm{GeV})$ and for the bottom quark the running mass $m_{b}\left(m_{H}=\right.$ $125.7 \mathrm{GeV})=2.763 \mathrm{GeV}$ is used. 


\section{CHAPTER 6. CONCLUSIONS}

process could be applied to other processes at either the LHC or a future $e^{+} e^{-}$collider. Projected sensitivities were estimated for high luminosity LHC scenarios and future colliders in Chapter 5. These projections suggest that other Higgs processes, such as $q \bar{q} \rightarrow Z H$ or $q \bar{q} \rightarrow H q \bar{q}$, will play an important role in the campaign for precision measurements of Higgs properties.

Other mechanisms for electroweak symmetry breaking include models in which the Higgs is composite. Measuring all of the HZZ amplitude parameters may one day provide hints of compositeness. However, it is necessary to use more advanced techniques in order to measure all parameters. Multidimensional fits provide the necessary flexibility to do so and are a natural evolution of the MELA technique.

The MELA techniques have provided immense utility to the high energy physics community. These tools have been used to discover and characterize the $126 \mathrm{GeV}$ Higgs-like resonance both at CMS and ATLAS [94]. The property measurements made have helped to shape our understanding of the role this resonance plays in nature and whether new physics is involved in its interactions with the SM fields. Even in the next generation of experiments, the MELA techniques will continue to provide a framework for performing high precision measurements and, hopefully, one day help us to better understand the universe we live in. 


\section{Bibliography}

[1] S. Bolognesi, Y. Gao, A. V. Gritsan, K. Melnikov, M. Schulze et al., "On the spin and parity of a single-produced resonance at the LHC," Phys.Rev., vol. D86, p. 095031, 2012.

[2] S. Glashow, "Partial Symmetries of Weak Interactions," Nucl.Phys., vol. 22, pp. $579-588,1961$.

[3] P. W. Anderson, "Plasmons, Gauge Invariance, and Mass," Phys.Rev., vol. 130, pp. 439-442, 1963.

[4] P. W. Higgs, "Broken Symmetries and the Masses of Gauge Bosons," Phys.Rev.Lett., vol. 13, pp. 508-509, 1964.

[5] F. Englert and R. Brout, "Broken Symmetry and the Mass of Gauge Vector Mesons," Phys.Rev.Lett., vol. 13, pp. 321-323, 1964.

[6] P. W. Higgs, "Broken symmetries, massless particles and gauge fields," Phys.Lett., vol. 12, pp. 132-133, 1964. 


\section{BIBLIOGRAPHY}

[7] G. Guralnik, C. Hagen, and T. Kibble, "Global Conservation Laws and Massless Particles," Phys.Rev.Lett., vol. 13, pp. 585-587, 1964.

[8] S. Weinberg, "A Model of Leptons," Phys.Rev.Lett., vol. 19, pp. 1264-1266, 1967.

[9] A. Salam, "Weak and Electromagnetic Interactions," Conf.Proc., vol. C680519, pp. $367-377,1968$.

[10] F. Hasert, H. Faissner, W. Krenz, J. Von Krogh, D. Lanske et al., "Search for elastic muon-neutrino electron scattering," Phys.Lett., vol. B46, pp. 121-124, 1973.

[11] F. Hasert et al., "Observation of Neutrino Like Interactions Without Muon Or Electron in the Gargamelle Neutrino Experiment," Phys.Lett., vol. B46, pp. 138$140,1973$.

[12] — - "Observation of Neutrino Like Interactions without Muon or Electron in the Gargamelle Neutrino Experiment," Nucl.Phys., vol. B73, pp. 1-22, 1974.

[13] G. Brianti and E. Gabathuler, "Intermediate vector bosons. Production and identification at the CERN proton anti-proton collider," Europhys. News, vol. 14, pp. 1-5, 1983.

[14] G. Arnison et al., "Experimental Observation of Lepton Pairs of Invariant Mass Around $95-\mathrm{GeV} / c^{2}$ at the CERN SPS Collider," Phys.Lett., vol. B126, pp. 398410, 1983 


\section{BIBLIOGRAPHY}

[15] R. Barate et al., "Search for the standard model Higgs boson at LEP," Phys.Lett., vol. B565, pp. 61-75, 2003.

[16] AlEPH, CDF, D0, DELPHI, L3, OPAL, SLD, L. E. W. Group, T. E. W. Group, S. E. W. Group, and H. F. Group, "Precision Electroweak Measurements and Constraints on the Standard Model," SLAC-PUB-14301, CERN-PH-EP2008-020, FERMILAB-TM-2420-E, LEPEWWG-2008-01, TEVEWWG-200801, ALEPH-2008-001-PHYSICS-2008-001, CDF-NOTE-9610, D0-NOTE-5802, DELPHI-2008-001-PHYS-950, L-NOTE-2834, OPAL-PR428, 2008.

[17] T. Aaltonen et al., "Combination of Tevatron searches for the standard model Higgs boson in the $W^{+} W^{-}$decay mode," Phys.Rev.Lett., vol. 104, p. 061802 , 2010.

[18] M. Sullivan, J. Guy, A. Conley, N. Regnault, P. Astier et al., "SNLS3: Constraints on Dark Energy Combining the Supernova Legacy Survey Three Year Data with Other Probes," Astrophys.J., vol. 737, p. 102, 2011.

[19] A. Sakharov, "Violation of CP Invariance, c Asymmetry, and Baryon Asymmetry of the Universe," Pisma Zh.Eksp.Teor.Fiz., vol. 5, pp. 32-35, 1967.

[20] C. Jarlskog, "Commutator of the Quark Mass Matrices in the Standard Electroweak Model and a Measure of Maximal CP Violation," Phys.Rev.Lett., vol. 55, p. 1039, 1985. 


\section{BIBLIOGRAPHY}

[21] M. Shaposhnikov, "Possible Appearance of the Baryon Asymmetry of the Universe in an Electroweak Theory," JETP Lett., vol. 44, pp. 465-468, 1986.

[22] I. Aitchison, Supersymmetry in Particle Physics: An Elementary Introduction. Cambridge University Press, 2007.

[23] E. Witten, "Dynamical Breaking of Supersymmetry," Nucl.Phys., vol. B188, p. $513,1981$.

[24] M. Veltman, "The Infrared - Ultraviolet Connection," Acta Phys.Polon., vol. B12, p. 437, 1981.

[25] R. K. Kaul, "Gauge Hierarchy in a Supersymmetric Model," Phys.Lett., vol. B109, p. 19, 1982.

[26] J. Shu and Y. Zhang, "Impact of a CP Violating Higgs: from LHC to Baryogenesis," CAS-KITPC-ITP-365, CALT-68-2926, 2013.

[27] L. Randall and R. Sundrum, "A Large mass hierarchy from a small extra dimension," Phys.Rev.Lett., vol. 83, pp. 3370-3373, 1999.

[28] G. Bayatian et al., "CMS physics: Technical design report," CERN-LHCC-2006001, CMS-TDR-008-1, 2006.

[29] —_ "CMS technical design report, volume II: Physics performance," J.Phys., vol. G34, pp. 995-1579, 2007. 


\section{BIBLIOGRAPHY}

[30] L. Borrello, "The CMS silicon strip tracker performance using cosmic ray data," Nucl.Instrum.Meth., vol. A623, pp. 153-155, 2010.

[31] V. Karimaki, T. Lampen, and F. Schilling, "The HIP algorithm for track based alignment and its application to the CMS pixel detector," CERN-CMS-NOTE2006-018, 2006.

[32] D. Brown, A. Gritsan, Z. Guo, and D. Roberts, "Local Alignment of the BABAR Silicon Vertex Tracking Detector," Nucl.Instrum.Meth., vol. A603, pp. 467-484, 2009.

[33] S. Dittmaier et al., "Handbook of LHC Higgs Cross Sections: 1. Inclusive Observables," CERN-2011-002, 2011.

[34] I. Anderson, S. Bolognesi, F. Caola, Y. Gao, A. V. Gritsan et al., "Constraining anomalous HVV interactions at proton and lepton colliders," 2013.

[35] Y. Gao, A. V. Gritsan, Z. Guo, K. Melnikov, M. Schulze et al., "Spin determination of single-produced resonances at hadron colliders," Phys.Rev., vol. D81, p. $075022,2010$.

[36] J. S. Gainer, K. Kumar, I. Low, and R. Vega-Morales, "Improving the sensitivity of Higgs boson searches in the golden channel," JHEP, vol. 1111, p. 027, 2011.

[37] Y. Chen, N. Tran, and R. Vega-Morales, "Scrutinizing the Higgs Signal and Background in the $2 e 2 \mu$ Golden Channel," JHEP, vol. 1301, p. 182, 2013. 


\section{BIBLIOGRAPHY}

[38] P. Nason, "A New method for combining NLO QCD with shower Monte Carlo algorithms," JHEP, vol. 0411, p. 040, 2004.

[39] S. Frixione, P. Nason, and C. Oleari, "Matching NLO QCD computations with Parton Shower simulations: the POWHEG method," JHEP, vol. 0711, p. 070, 2007.

[40] S. Alioli, P. Nason, C. Oleari, and E. Re, "NLO vector-boson production matched with shower in POWHEG," JHEP, vol. 0807, p. 060, 2008.

[41] J. Alwall, P. Demin, S. de Visscher, R. Frederix, M. Herquet et al., "MadGraph/MadEvent v4: The New Web Generation," JHEP, vol. 0709, p. 028, 2007.

[42] M. L. Mangano, M. Moretti, F. Piccinini, R. Pittau, and A. D. Polosa, "ALPGEN, a generator for hard multiparton processes in hadronic collisions," JHEP, vol. 0307, p. 001, 2003.

[43] T. Sjostrand, S. Mrenna, and P. Z. Skands, "PYTHIA 6.4 Physics and Manual," JHEP, vol. 0605, p. 026, 2006.

[44] S. Kretzer, H. Lai, F. Olness, and W. Tung, "CTEQ6 parton distributions with heavy quark mass effects," Phys.Rev., vol. D69, p. 114005, 2004.

[45] H.-L. Lai, M. Guzzi, J. Huston, Z. Li, P. M. Nadolsky et al., "New parton distributions for collider physics," Phys.Rev., vol. D82, p. 074024, 2010. 


\section{BIBLIOGRAPHY}

[46] S. Agostinelli et al., "GEANT4: A Simulation toolkit," Nucl.Instrum.Meth., vol. A506, pp. 250-303, 2003.

[47] S. Chatrchyan et al., "Search for a Higgs boson in the decay channel $H \rightarrow$ $Z Z^{(*)} \rightarrow q \bar{q} \ell^{-} l^{+}$in $p p$ collisions at $\sqrt{s}=7$ TeV," JHEP, vol. 1204, p. 036, 2012.

[48] M. Cacciari, G. P. Salam, and G. Soyez, "The anti- $k_{t}$ jet clustering algorithm," JHEP, vol. 0804, p. 063, 2008.

[49] —_, "FastJet User Manual," Eur.Phys.J., vol. C72, p. 1896, 2012.

[50] S. Chatrchyan et al., "Determination of Jet Energy Calibration and Transverse Momentum Resolution in CMS," JINST, vol. 6, p. P11002, 2011.

[51] M. Cacciari, G. P. Salam, and G. Soyez, "The Catchment Area of Jets," JHEP, vol. 0804, p. 005, 2008.

[52] S. Chatrchyan et al., "Commissioning of b-jet identification with pp collisions at $\sqrt{s}=7 \mathrm{TeV}, " C M S-P A S-B T V-10-001,2010$.

[53] — - "Measurement of btagging efficiency using ttbar events," CMS-PAS-BTV11-003, 2012.

[54] — - "Missing transverse energy performance of the CMS detector," JINST, vol. 6, p. P09001, 2011. 


\section{BIBLIOGRAPHY}

[55] C. Anastasiou, R. Boughezal, and F. Petriello, "Mixed QCD-electroweak corrections to Higgs boson production in gluon fusion," JHEP, vol. 0904, p. 003, 2009

[56] D. de Florian and M. Grazzini, "Higgs production through gluon fusion: Updated cross sections at the Tevatron and the LHC," Phys.Lett., vol. B674, pp. 291-294, 2009.

[57] J. Baglio and A. Djouadi, "Higgs production at the LHC," JHEP, vol. 1103, p. $055,2011$.

[58] A. Djouadi, M. Spira, and P. Zerwas, "Production of Higgs bosons in proton colliders: QCD corrections," Phys.Lett., vol. B264, pp. 440-446, 1991.

[59] S. Dawson, "Radiative corrections to Higgs boson production," Nucl.Phys., vol. B359, pp. 283-300, 1991.

[60] M. Spira, A. Djouadi, D. Graudenz, and P. Zerwas, "Higgs boson production at the LHC," Nucl.Phys., vol. B453, pp. 17-82, 1995.

[61] R. V. Harlander and W. B. Kilgore, "Next-to-next-to-leading order Higgs production at hadron colliders," Phys.Rev.Lett., vol. 88, p. 201801, 2002.

[62] C. Anastasiou and K. Melnikov, "Higgs boson production at hadron colliders in NNLO QCD," Nucl.Phys., vol. B646, pp. 220-256, 2002. 


\section{BIBLIOGRAPHY}

[63] V. Ravindran, J. Smith, and W. L. van Neerven, "NNLO corrections to the total cross-section for Higgs boson production in hadron hadron collisions," Nucl.Phys., vol. B665, pp. 325-366, 2003.

[64] S. Catani, D. de Florian, M. Grazzini, and P. Nason, "Soft gluon resummation for Higgs boson production at hadron colliders," JHEP, vol. 0307, p. 028, 2003.

[65] S. Actis, G. Passarino, C. Sturm, and S. Uccirati, "NLO Electroweak Corrections to Higgs Boson Production at Hadron Colliders," Phys.Lett., vol. B670, pp. 1217, 2008.

[66] U. Aglietti, R. Bonciani, G. Degrassi, and A. Vicini, "Two loop light fermion contribution to Higgs production and decays," Phys.Lett., vol. B595, pp. 432441, 2004.

[67] M. Ciccolini, A. Denner, and S. Dittmaier, "Strong and electroweak corrections to the production of Higgs +2 jets via weak interactions at the LHC," Phys.Rev.Lett., vol. 99, p. 161803, 2007.

[68] — - "Electroweak and QCD corrections to Higgs production via vector-boson fusion at the LHC," Phys.Rev., vol. D77, p. 013002, 2008.

[69] T. Figy, C. Oleari, and D. Zeppenfeld, "Next-to-leading order jet distributions for Higgs boson production via weak boson fusion," Phys.Rev., vol. D68, p. 073005, 2003. 


\section{BIBLIOGRAPHY}

[70] K. Arnold, M. Bahr, G. Bozzi, F. Campanario, C. Englert et al., "VBFnLO: A Parton level Monte Carlo for processes with electroweak bosons," Comput.Phys.Commun., vol. 180, pp. 1661-1670, 2009.

[71] P. Bolzoni, F. Maltoni, S.-O. Moch, and M. Zaro, "Higgs production via vectorboson fusion at NNLO in QCD," Phys.Rev.Lett., vol. 105, p. 011801, 2010.

[72] T. Figy, S. Palmer, and G. Weiglein, "Higgs Production via Weak Boson Fusion in the Standard Model and the MSSM," JHEP, vol. 1202, p. 105, 2012.

[73] A. Djouadi, J. Kalinowski, and M. Spira, "HDECAY: A Program for Higgs boson decays in the standard model and its supersymmetric extension," Comput.Phys.Commun., vol. 108, pp. 56-74, 1998.

[74] A. Bredenstein, A. Denner, S. Dittmaier, and M. Weber, "Precise predictions for the Higgs-boson decay $H \rightarrow W W / Z Z \rightarrow 4$ leptons," Phys.Rev., vol. D74, p. 013004, 2006.

[75] — _ "Radiative corrections to the semileptonic and hadronic Higgs-boson decays $H \rightarrow W W / Z Z \rightarrow 4$ fermions," JHEP, vol. 0702, p. 080, 2007.

[76] S. Chatrchyan et al., "Combined results of searches for the standard model Higgs boson in $p p$ collisions at $\sqrt{s}=7 \mathrm{TeV}$," Phys.Lett., vol. B710, pp. 26-48, 2012.

[77] A. L. Read, "Presentation of search results: The $C L_{s}$ technique," J.Phys., vol. G28, pp. 2693-2704, 2002. 


\section{BIBLIOGRAPHY}

[78] G. Passarino, C. Sturm, and S. Uccirati, "Higgs Pseudo-Observables, Second Riemann Sheet and All That," Nucl.Phys., vol. B834, pp. 77-115, 2010.

[79] S. Goria, G. Passarino, and D. Rosco, "The Higgs Boson Lineshape," Nucl.Phys., vol. B864, pp. 530-579, 2012.

[80] N. Kauer, "Inadequacy of zero-width approximation for a light Higgs boson signal," Mod.Phys.Lett., vol. A28, p. 1330015, 2013.

[81] G. Passarino, "Higgs Interference Effects in $g g \rightarrow Z Z$ and their Uncertainty," JHEP, vol. 1208, p. 146, 2012.

[82] T. Binoth, N. Kauer, and P. Mertsch, "Gluon-induced QCD corrections to $p p \rightarrow$ $Z Z \rightarrow \ell \bar{\ell} \ell^{\prime} \bar{\ell}^{\prime}, "$ arXiv:0807.0024, p. 142, 2008.

[83] H.-L. Lai, J. Huston, Z. Li, P. Nadolsky, J. Pumplin et al., "Uncertainty induced by QCD coupling in the CTEQ global analysis of parton distributions," Phys.Rev., vol. D82, p. 054021, 2010.

[84] J. Allison, K. Amako, J. Apostolakis, H. Araujo, P. Dubois et al., "Geant4 developments and applications," IEEE Trans.Nucl.Sci., vol. 53, p. 270, 2006.

[85] M. Cacciari and G. P. Salam, "Pileup subtraction using jet areas," Phys.Lett., vol. B659, pp. 119-126, 2008.

[86] S. Chatrchyan et al., "Properties of the Higgs-like boson in the decay $H \rightarrow$ $Z Z \rightarrow 4 \ell$ in pp collisions at $\sqrt{s}=7$ and $8 \mathrm{TeV}, " C M S-P A S-H I G-13-002,2013$. 


\section{BIBLIOGRAPHY}

[87] M. Botje, J. Butterworth, A. Cooper-Sarkar, A. de Roeck, J. Feltesse et al., "The PDF4LHC Working Group Interim Recommendations," arXiv:1101.0538, 2011.

[88] S. Alekhin, S. Alioli, R. D. Ball, V. Bertone, J. Blumlein et al., "The PDF4LHC Working Group Interim Report,” 2011.

[89] G. Cowan, K. Cranmer, E. Gross, and O. Vitells, "Asymptotic formulae for likelihood-based tests of new physics," Eur.Phys.J., vol. C71, p. 1554, 2011.

[90] M. Koratzinos, A. Blondel, R. Aleksan, O. Brunner, A. Butterworth et al., "TLEP: A High-Performance Circular $e^{+} e^{-}$Collider to Study the Higgs Boson," arXiv:1305.6498, 2013.

[91] T. Behnke, J. E. Brau, B. Foster, J. Fuster, M. Harrison et al., "The International Linear Collider Technical Design Report - Volume 1: Executive Summary," ILC-REPORT-2013-040, ANL-HEP-TR-13-20, BNL-100603-2013-IR, IRFU13-59, CERN-ATS-2013-037, COCKCROFT-13-10, CLNS-13-2085, DESY13-062, FERMILAB-TM-2554, IHEP-AC-ILC-2013-001, INFN-13-04-LNF, JAI-2013-001，JINR-E9-2013-35，JLAB-R-2013-01，KEK-REPORT-2013-1, KNU-CHEP-ILC-2013-1, LLNL-TR-635539, SLAC-R-1004, ILC-HIGRADEREPORT-2013-003, SLACcitation =, 2013.

[92] S. Chatrchyan et al., "Combination of standard model Higgs boson searches and measurements of the properties of the new boson with a mass near $125 \mathrm{GeV}$," CMS-PAS-HIG-13-005, 2013. 


\section{BIBLIOGRAPHY}

[93] G. Aad et al., "Study of the spin of the new boson with up to $25 \mathrm{fb}^{-1}$ of ATLAS data," ATLAS-CONF-2013-040, ATLAS-COM-CONF-2013-048, 2013.

[94] — "Measurements of the properties of the Higgs-like boson in the four lepton decay channel with the ATLAS detector using $25 \mathrm{fb}^{-1}$ of proton-proton collision data," ATLAS-CONF-2013-013, ATLAS-COM-CONF-2013-018, 2013. 


\section{Vita}

Andrew Whitbeck received a Bachelors of Science degree in Physics and a Bachelor of Arts degree in Mathematics from the University of Rochester in 2007. He was awarded the Stoddard Prize for his senior thesis, "A Three-body partial decay width in the Littlest Higgs model," under the guidance of Lynne H. Orr. In 2007, Andrew started his Ph.D. at Johns Hopkins University and joined the CMS collaboration under the tutelage of Andrei Gritsan in 2009. Andrew will continue his work with the CMS collaboration at the Fermi National Accelerator Laboratory as a postdoctoral researcher.

\section{Education}

- Ph.D. Experimental Particle Physics, Johns Hopkins University, September 2013.

- B.S. Physics, with honors, University of Rochester, 2007. 
VITA

- B.A. Mathematics, University of Rochester, 2007.

\section{Honors and Awards}

- Rencontres de Moriond QCD Travel Grant, 2013.

- E.J. Rhee Travel Grant, 2011.

- National Science Foundation (NSF) US LHC Graduate Student Support Award, 2010-2011.

- Stoddard Prize for best senior thesis, University of Rochester, 2007.

\section{Employment}

- Research Assistant to Andrei Gritsan, Johns Hopkins University, CMS Collaboration, 2009 - present.

- Research Assistant to Lynne H. Orr, University of Rochester, 2006 - 2007.

- Research Assistant to Douglas Cline, University of Rochester, June 2006 - August 2006 .

- Research Assistant to Doug Higinbotham, Jefferson National Laboratory, June 2005 - August 2005. 
VITA

\section{Publications}

- "Study of the mass and spin-parity of the Higgs boson candidate via its decays to Z boson pairs", The CMS Collaboration, Phys. Rev. Lett. 110.

- "Search for a narrow, spin-2 resonance decaying to a pair of Z bosons in the $q \bar{q} \ell^{+} \ell^{-}$final state", The CMS Collaboration, arXiv:1209.3807, submitted to PLB.

- "On the spin and parity of a single-produced resonance at the LHC", Bolognesi, Sara et al. Phys. Rev. D 86.

- "Observation of a new boson at a mass of $125 \mathrm{GeV}$ with the CMS experiment at the LHC", The CMS Collaboration, arXiv:1207.7235 [hep-ex].

- "Combined results of searches for the standard model Higgs boson in pp collisions at $\sqrt{s}=7$ TeV", The CMS Collaboration, Phys. Lett. B 710 (2012) $26-48$.

- "Search for a Higgs boson in the decay channel $H \rightarrow Z Z^{(*)} \rightarrow q \bar{q} \ell^{-} \ell^{+}$in pp collisions at $\sqrt{s}=7 \mathrm{TeV}$ ", The CMS Collaboration, JHEP 1204 (2012) 036 .

- "Search for a fermiophobic Higgs boson in pp collisions at $\sqrt{s}=7 \mathrm{TeV}$ ", The CMS Collaboration, arXiv:1207.1130 [hep-ex] CMS-HIG-12-009, CERNPH-EP-2012-174. 
VITA

- "Search for the standard model Higgs boson in the decay channel $H \rightarrow Z Z \rightarrow 4$ leptons in pp collisions at $\sqrt{s}=7 \mathrm{TeV}$ ", The CMS Collaboration, Phys. Rev. Lett. 108 (2012) 111804.

- "Coulomb excitation of the proton-dripline nucleus Na-20", Schumaker, M.A. et al. Phys. Rev. C 80 (2009) 044325, Erratum-ibid. C82 (2010) 069902.

- "Coulomb excitation of radioactive Na-21 and its stable mirror Ne-21", Schumaker, M.A. et al. Phys. Rev. C 78 (2008) 044321.

- "Decays of the Littlest Higgs ZH and the Onset of Strong Dynamics", Boersma, John, Whitbeck, Andrew, Phys. Rev. D 77 (2008) 055012.

- "Precision Measurements of the Nucleon Strange Form Factors at $\mathrm{Q}^{2} \sim 0.1$ GeV²", HAPPEX Collaboration (Acha, A. et al.) Phys. Rev. Lett. 98 (2007)

\section{CMS Physics Analysis Summaries}

- "Properties of the Higgs-like boson in the decay $\mathrm{H} \rightarrow Z Z \rightarrow 4 \ell$ in pp collisions at $\sqrt{s}=7$ and 8 TeV.", CMS AN-13-002, February 2013.

- "Evidence for a new state in the search for the standard model Higgs boson in the $H \rightarrow Z Z \rightarrow 4$ leptons channel in pp collisions at $\sqrt{s}=7$ and $8 \mathrm{TeV}$ ", The CMS Collaboration, CMS-PAS-HIG-12-016. 
VITA

- "Search for the standard model Higgs Boson in the decay channel $H \rightarrow Z Z^{(*)} \rightarrow$ $q \bar{q} \ell^{-} \ell^{+}$at CMS", The CMS Collaboration, CMS-PAS-HIG-11-027.

- "Search for the standard model Higgs Boson in the decay channel $H \rightarrow Z Z \rightarrow$ $\ell \ell q q$ at CMS", CMS Collaboration, The CMS-PAS-HIG-11-017.

- "Search for the standard model Higgs Boson in the decay channel $H \rightarrow Z Z \rightarrow$ $\ell \ell q q$ at CMS", CMS Collaboration, The CMS-PAS-HIG-11-006.

\section{CMS Analysis Notes}

- "Search for a narrow spin-2 resonance decaying to $\mathrm{Z}$ vector bosons in the semileptonic final state", CMS AN-12-017, June 2012.

- "Search for the standard model Higgs boson in the decay channel $H \rightarrow Z Z \rightarrow 4 \ell$ in pp collisions", CMS AN-12-141, June 2012.

- "Search for a SM Higgs or BSM Boson $H \rightarrow Z Z^{(*)} \rightarrow\left(q^{-} q^{+}\right)\left(\ell^{-} \ell^{+}\right)$", CMS AN -2011/388, December 2011.

- "Search for a Higgs boson in the decay channel $H \rightarrow Z Z^{(*)} \rightarrow 4 \ell^{\text {", }}$ CMS AN11-387, December 2011.

- "Search for a SM Higgs or BSM Boson $H \rightarrow Z Z \rightarrow\left(\ell^{-} \ell^{+}\right)\left(q^{-} q^{+}\right)$", CMS AN-2011-100, June 2011. 
VITA

- "Angular Analysis of Resonances $p p \rightarrow X \rightarrow Z Z$ ", CMS AN-2010-351, November 2010 .

\section{Conference Presentations}

- "Properties of the Higgs-like boson with CMS", Johns Hopkins Particle Physics Seminar, March 2013.

- "Higgs Property Measurements", US CMS Weekly Meeting, March 2013.

- "Higgs Candidate Property Measurements with the Compact Muon Solenoid", Rencontres de Moriond, La Thuile, Italy, March 2013.

- "Characterization of a single-produced resonance at the LHC: Prospects for 2012 and Beyond", Phenomenology 2012 Symposium, University of Pittsburgh, May 2012.

- " $H \rightarrow Z Z \rightarrow 2 l 2 q$ ", CMS Approval, August 2011.

- "Higgs properties analyses in ATLAS and CMS", Implications of LHC results for TeV-scale physics, CERN, August 2011.

- "The search for the SM Higgs $\rightarrow Z Z$ with hadronic Z decay", US CMS Weekly Meeting, June 2011. 
VITA

- "Pre-approval talk: $H \rightarrow Z Z \rightarrow 2 l 2 j "$, CMS Higgs PAG Pre-approval, June 2011.

- "Discovery prospects for Higgs $\rightarrow Z Z \rightarrow 2 l 2 j$ and implications for other resonances" APS April Meeting, Anaheim CA, May 2011.

\section{Outreach}

- Johns Hopkins Physics Fair, Baltimore MD, USA, 2008, 2009, 2010, 2011, and 2012

- "The Science of the Large Hadron Collider", USA Science and Engineering Festival, Washington DC, USA, October 2010 and April 2012.

- "High Energy Physics", Loch Raven HS, Baltimore MD, USA, February 2013. 